\title{
ANTONIO LUÍS VENEZUELA
}

\section{MODELAGEM ANALÍTICO-NUMÉRICA DO ESCOAMENTO LAMINAR CONVECTIVO EM TUBOS ASSOCIADA À FILTRAÇÃO TANGENCIAL}

Tese apresentada à Escola de Engenharia de São Carlos da Universidade de São Paulo para obtenção do Título de Doutor em Engenharia Mecânica.

Área de Concentração: Térmicas e Fluidos

Orientador: Prof. Dr. Sérgio Rodrigues Fontes 

Para minha esposa Mara. 



\section{Agradecimentos}

Ao meu orientador Prof. Dr. Sergio Rodrigues Fontes pela motivação, confiança, parceria e companheirismo para realização deste trabalho. Agradeço pelas suas sugestões e ajuda na escolha do tema aqui desenvolvido. Agradeço pela forma sempre serena e objetiva que me conduziu na procura de soluções dos vários problemas e dificuldades que encontrei durante o desenvolvimento deste trabalho.

Ao Prof. Dr. Jésus Salvador Perez-Guerrero pela motivação, pela importante ajuda na teoria e na implementação computacional da técnica da transformada integral. Agradeço ainda pelo tempo que disponibilizou para as várias reuniões realizadas no Rio de Janeiro, durante o desenvolvimento deste trabalho.

Ao Prof. Dr. Emanuel Macedo por ter fornecido importantes informações teóricas sobre a técnica da transformada integral, durante o ENCIT 2004.

Aos professores da banca de qualificação: Prof. Dr. Reginaldo J. Napolitano e Prof. Dr. Fernando E. Milioli.

À minha esposa Mara pelo carinho, apoio, motivação e paciência.

À amiga e colega de departamento Juliana Maria Silva pela ajuda e discussões construtivas durante todos estes anos. 
À minha sogra Idalva G. Fernandes, sogro Antonio Fernandes (in memoriam), e meu cunhado Marco A. Fernandes pela confiança e motivação e sua esposa Edilene G. Fernandes que, além da confiança, colaborou com traduções de alguns trabalhos.

Aos meus familiares: Francisco Venezuela e Ana Rodrigues Venezuela (meus pais), Lúcia, Isidoro e Aparecida (meus irmãos), cunhados e sobrinhos pela confiança.

Aos colegas do departamento Ivonete Ávila (colaborou com a formatação deste trabalho), Cecília Leiva, Marcelo Evaristo, Renata Haneda, Roberta Del Colle, Ernesto Beck, Viviane M. Silva e Carlos pela agradável convivência e também ao colega Ricardo Chebib.

Ao casal amigo Marcelo e Fabiani Noguti pela ajuda e por manter as portas de sua casa sempre abertas para mim. 


\section{Resumo}

Venezuela, A. L. Modelagem Analítico-Numérica do Escoamento Laminar Convectivo em Tubos Associada à Filtração Tangencial. 2008, 175p. Tese (Doutorado). Escola de Engenharia de São Carlos, Universidade de São Paulo, São Carlos, 2008.

Nesta tese de doutorado é utilizada a técnica híbrida analítico-numérica, conhecida internacionalmente por GITT (Generalized Integral Transform Technique), para modelagem e simulação da equação de conservação das espécies químicas, na investigação do escoamento laminar incompressível, newtoniano e permanente em tubos permeáveis. O escoamento é aplicado ao processo de filtração tangencial com membranas e foram realizados dois estudos relacionados à equação convectiva-difusiva elíptica e parabólica, para as quais são utilizadas as mesmas condições de fronteira. Na modelagem a velocidade na parede permeável é considerada uniforme e os perfis de velocidade para a região de entrada do escoamento são obtidos da literatura. O modelo matemático utiliza originalmente uma expressão para a espessura da camada limite de concentração, com uma metodologia que determina a taxa assintótica, com a qual se estabelece a espessura da camada de concentração. Os resultados são apresentados com análise de convergência através de tabelas e com gráficos para o fluxo transmembrana local e médio, a correlação de Sherwood e a espessura da camada limite de concentração e ainda são comparados com outros resultados e metodologias reportadas na literatura.

Palavras-Chave: Modelagem matemática; Transformada integral; Tubo permeável; Filtração tangencial; Transferência de massa. 



\section{Abstract}

Venezuela, A. L. Analytical-Numerical Modeling of Convective Laminar Flow in Tubes Associated with Cross-Flow. 2008, 175p. Thesis (Doctoral). Escola de Engenharia de São Carlos, Universidade de São Paulo, São Carlos, 2008.

In this doctoral thesis, the analytical-numerical hybrid technique, internationally known as GITT (Generalized Integral Transform Technique), is used for the modeling and simulation of the equation of chemical species conservation, in the investigation of the incompressible, Newtonian and permanent laminar flow in permeable tubes. The flow is applied to the crossflow process with membranes and two studies related to the elliptic and parabolic convectivediffusive equation were accomplished, for which the same boundary conditions are used. In the modeling, the velocity on the permeable wall is considered uniform and the velocity profiles for the entrance region flow are obtained from the literature. The mathematical model originally uses an expression for the concentration boundary layer thickness, with a methodology that determines the asymptotic ratio, establishing the concentration boundary layer thickness. The results are presented with convergence analysis through tables and with graphs for the mean local transmembrane flux, Sherwood correlation and the concentration boundary layer thickness, and they are also compared with other results and methodologies reported in the literature.

Keywords: Mathematical modeling, Integral transform, Permeable tube, Cross flow, Mass Transfer. 



\section{Lista de Figuras}

Figura 1. Princípio da filtração tangencial, onde temos o fluxo de filtrado (ou fluxo transmembrana), o fluxo tangencial e do retentado (Adaptado de RIPPERGER e ALTMANN, 2002)......

Figura 2. Representação esquemática da pressão transmembrana, $\Delta \mathrm{p}$, da pressão de entrada, $\mathrm{p}_{0}$, da pressão do permeado (ou exterior), $\mathrm{p}_{\text {ext, }}$, a pressão local $\mathrm{p}(\mathrm{x})$. 42

Figura 3. Representação esquemática de um poro cilíndrico, bem como as indicações da concentração na parede, $\mathrm{c}_{\mathrm{w}}$, a concentração de filtrado, $\mathrm{c}_{\mathrm{f}}$, o raio do poro, $\mathrm{r}_{\mathrm{po}}$, a espessura da membrana, $\delta_{\mathrm{m}}$, a velocidade na parede, $\mathrm{v}_{\mathrm{w}}$, e o fluxo de soluto dentro do poro, $\mathrm{N}_{\mathrm{S}}$.

Figura 4. Diagrama esquemático da concentração polarizada e da camada limite de concentração, de espessura $\delta$, em filtração tangencial. Um gradiente de concentração é produzido através da camada limite de concentração. Temos também a indicação do fluxo transmembrana e do escoamento central.....

Figura 5. Desenho esquemático do balanço de massa na superfície permeável, onde " $\hat{n}$ " é o vetor unitário e normal à membrana, " $\vec{v}$ " o vetor velocidade transversal (de intensidade " $\mathrm{v}_{\mathrm{w}}$ ", paralelo e oposto ao vetor normal). O fluxo transmembrana, J, também é paralelo ao vetor velocidade " $\vec{v}$ ". Indicamos " $u$ " e "v", respectivamente, sendo a velocidade longitudinal e velocidade transversal. 50

Figura 6. Esquema da condição de contorno e entrada de um tubo permeável de comprimento "L" e raio " $\mathrm{R}_{0}$ ", para um escoamento em regime estacionário e laminar de um fluido incompressível e newtoniano.

Figura 7. Representação do escoamento laminar completamente desenvolvido em tubo com superfície permeável.

Figura 8. Representação esquemática do perfil de concentração em tubo permeável de raio $\mathrm{R}_{0}$, cuja concentração de entrada é $\mathrm{c}_{0}$. 
Figura 9. Espessura da camada limite de concentração variando com posição axial " $\tilde{x}$ ” e a representação da reta assintótica.

Figura 10. Representação esquemática da metodologia da modelagem que envolve 9 passos. No passo (2), a espessura da camada limite de concentração polarizada, $\delta(\iota, X)$, e a concentração na parede, $\mathrm{C}_{\mathrm{w}}(\mathrm{X})$, são avaliadas para uma taxa assintótica, $\iota$, inicial, as quais serão ajustadas no final do processo segundo o critério do passo (7)....... 95

Figura 11. Esquema para o intervalo $\mathrm{I}=\left[(1-\varepsilon) \mathrm{V}_{\mathrm{w}},(1+\varepsilon) \mathrm{V}_{\mathrm{w}}\right]$ relacionando " $\mathrm{J}_{\mathrm{m}}$ " $\mathrm{e}$ " $\mathrm{V}_{\mathrm{w}}$ ". 97

Figura 12. (Equação convectiva-difusiva elíptica) Efeito do número de Reynolds sobre o perfil de concentração em função da posição axial adimensional $\left(\mathrm{x} / \mathrm{R}_{0}\right)$ próximo à superfície permeável $(\mathrm{R}=0,98)$ para $\mathrm{Re}_{\mathrm{w}}=0,005, \mathrm{Sc}=2500, \mathrm{R}_{0}=3,010^{-3} \mathrm{~m}$ e $\mathrm{N}=35$.

Figura 13. (Equação convectiva-difusiva parabólica) Efeito do número de Reynolds sobre o perfil de concentração em função da posição axial adimensional $\left(\mathrm{x} / \mathrm{R}_{0}\right)$ próximo à superfície permeável $(\mathrm{R}=0,98)$ para $\mathrm{Re}_{\mathrm{w}}=0,005, \mathrm{Sc}=2500, \mathrm{R}_{0}=3,010^{-3} \mathrm{~m}$ e $\mathrm{N}=46$. 108

Figura 14. (Equação convectiva-difusiva elíptica) Efeito do número de Reynolds de permeação sobre o perfil de concentração em função da posição axial adimensional $\left(\mathrm{x} / \mathrm{R}_{0}\right)$ próximo à superfície permeável $(\mathrm{R}=0,98)$ para $\mathrm{N}=35, \mathrm{Re}=700, \mathrm{Sc}=2500 \mathrm{e}$ $\mathrm{R}_{0}=3,010^{-3} \mathrm{~m}$. 108

Figura 15. (Equação convectiva-difusiva parabólica) Efeito do número de Reynolds de permeação sobre o perfil de concentração em função da posição axial adimensional $\left(\mathrm{x} / \mathrm{R}_{0}\right)$ próximo à superfície permeável $(\mathrm{R}=0,98)$ para $\mathrm{N}=46, \mathrm{Re}=700, \mathrm{Sc}=2500 \mathrm{e}$ $\mathrm{R}_{0}=3,010^{-3}$.

Figura 16. (Equação convectiva-difusiva elíptica) Efeito do número de Schmidt sobre o perfil de concentração em função da posição axial adimensional $\left(\mathrm{x} / \mathrm{R}_{0}\right)$ próximo à superfície permeável $(\mathrm{R}=0,98)$ para $\mathrm{N}=35, \mathrm{Re}=700, \mathrm{Re}_{\mathrm{w}}=0,005$ e $\mathrm{R}_{0}=3,010^{-3} \mathrm{~m} .109$

Figura 17. (Equação convectiva-difusiva parabólica) Efeito do número de Schmidt sobre o perfil de concentração em função da posição axial adimensional $\left(x / R_{0}\right)$ próximo à superfície permeável $(\mathrm{R}=0,98)$ para $\mathrm{Re}=700, \mathrm{Re}_{\mathrm{w}}=0,005, \mathrm{~N}=46$ e $\mathrm{R}_{0}=310^{-3} \mathrm{~m} . .110$

Figura 18. (Equação convectiva-difusiva elíptica) Efeito do número de Reynolds axial sobre o perfil do número de Sherwood local para $\mathrm{Sc}=2500, \mathrm{Re}_{\mathrm{w}}=0,005, \mathrm{R}_{0}=3,010^{-3} \mathrm{~m}$, $\mathrm{N}=35$. 
Figura 19. (Equação convectiva-difusiva parabólica) Efeito do número de Reynolds axial sobre o perfil do número de Sherwood local para $\mathrm{Sc}=2500, \mathrm{Re}_{\mathrm{w}}=0,005, \mathrm{~N}=46 \mathrm{e}$ $\mathrm{R}_{0}=3,010^{-3} \mathrm{~m}$.

Figura 20. (Equação convectiva-difusiva elíptica) Efeito do número de Reynolds de permeação sobre o perfil do número de Sherwood local para $\mathrm{Sc}=2500, \mathrm{Re}=700$, $\mathrm{R}_{0}=3,010^{-3} \mathrm{~m}, \mathrm{~N}=35$.

Figura 21. (Equação convectiva-difusiva parabólica) Efeito do número de Reynolds de permeação sobre o perfil do número de Sherwood local para $\mathrm{Sc}=2500, \mathrm{Re}=700$, $\mathrm{R}_{0}=3,010^{-3} \mathrm{~m}, \mathrm{~N}=46$.

Figura 22. (Equação convectiva-difusiva elíptica) Efeito do número de Schmidt sobre o perfil do número de Sherwood local para $\mathrm{Re}=700, \mathrm{Re}_{\mathrm{w}}=0,005, \mathrm{R}_{0}=3,010^{-3} \mathrm{~m}, \mathrm{~N}=35 \ldots 113$

Figura 23. (Equação convectiva-difusiva parabólica) Efeito do número de Schmidt sobre o perfil do número de Sherwood local para $R e=700, R_{\mathrm{w}}=0,005, \mathrm{R}_{0}=3,010^{-3} \mathrm{~m}$, $\mathrm{N}=46$.

Figura 24. Comparação entre as correlações de Gilland-Sherwood, sendo $\mathrm{R}_{0}=3,010^{-3} \mathrm{~m}$, $\mathrm{Re}=700, \mathrm{Sc}=800, \mathrm{Re}_{\mathrm{w}}=0,014$.

Figura 25. Comparação entre as correlações de Gilland-Sherwood, sendo $\mathrm{R}_{0}=3,010^{-3} \mathrm{~m}$, $\mathrm{Re}=200, \mathrm{Sc}=2500, \mathrm{Re}_{\mathrm{w}}=0,005$ 116

Figura 26. Comparações entre as correlações de Gilland-Sherwood, sendo $\mathrm{R}_{0}=3,010^{-3} \mathrm{~m}$, $\mathrm{Re}=200, \mathrm{Sc}=2500, \mathrm{Re}_{\mathrm{w}}=0,005$. 117

Figura 27. Comparações entre as correlações de Gilland-Sherwood, sendo $\mathrm{R}_{0}=3,010^{-3} \mathrm{~m}$, $\mathrm{Re}=700, \mathrm{Sc}=2500, \mathrm{Re}_{\mathrm{w}}=0,005$. 117

Figura 28. Comparações entre as correlações de Gilland-Sherwood, sendo $\mathrm{R}_{0}=3,010^{-3} \mathrm{~m}$, $\mathrm{Re}=700, \mathrm{Sc}=2500, \mathrm{Re}_{\mathrm{w}}=0.014$. 118

Figura 29. Comparações entre as correlações de Gilland-Sherwood, sendo $\mathrm{R}_{0}=3,010^{-3} \mathrm{~m}$, $\mathrm{Re}=700, \mathrm{Sc}=800, \mathrm{Re}_{\mathrm{w}}=0,014$.

Figura 30. Comparação entre os resultados deste trabalho com outro numérico da literatura para o fluxo transmembrana local, com $\mathrm{c}_{0}=8 \mathrm{~g} / \mathrm{l}, \Delta \mathrm{p}=4,910^{5} \mathrm{~Pa}, \mathrm{u}_{0}=0,216 \mathrm{~m} / \mathrm{s}$, $\mathrm{R}_{\mathrm{m}}+\mathrm{R}_{\mathrm{e}}=2,6710^{10} \mathrm{~Pa} \mathrm{~s} \mathrm{~m}^{-1}, \phi=6,8710^{5} \mathrm{~s} \mathrm{~m}^{-1}, \mathrm{v}_{\mathrm{w}}=1,3510^{-6} \mathrm{~m} \mathrm{~s}^{-1}$ e $\iota_{\max }=0,226 \ldots 120$

Figura 31. Comparação entre os resultados deste trabalho com outro numérico da literatura 
para a espessura da camada limite de concentração, com $\mathrm{c}_{0}=8 \mathrm{~g}^{-1}, \Delta \mathrm{p}=4,910^{5} \mathrm{~Pa}$, $\mathrm{R}_{\mathrm{m}}+\mathrm{R}_{\mathrm{e}}=2,6710^{10} \mathrm{~Pa} \mathrm{~s} \mathrm{~m}^{-1}, \mathrm{v}_{\mathrm{w}}=1,3510^{-6} \mathrm{~m} \mathrm{~s}^{-1}, \phi=6,8710^{5} \mathrm{~s} \mathrm{~m}^{-1}, \mathrm{u}_{0}=0,216 \mathrm{~m} \mathrm{~s}^{-1} \mathrm{e}$ $\iota_{\max }=0,226$.

Figura 32. Comparação entre os resultados deste trabalho com outro numérico para o perfil de concentração, com $\Delta \mathrm{p}=1,010^{5} \mathrm{~Pa}, \mathrm{R}_{\mathrm{m}}=2,810^{9} \mathrm{~Pa} \mathrm{~s} \mathrm{~m}^{-1}, \mathrm{R}_{\mathrm{e}}=0,110^{9} \mathrm{~Pa} \mathrm{~s} \mathrm{~m}^{-1}$, $\phi=0,9610^{5} \mathrm{~s} \mathrm{~m}^{-1}, \mathrm{R}_{0}=1,510^{-2} \mathrm{~m}, \mathrm{Sc}=14, \mathrm{Re}_{\mathrm{w}}=0,52, \mu=10^{-3} \mathrm{~kg} \mathrm{~m}^{-1} \mathrm{~s}^{-1}$ e $\rho=10^{3} \mathrm{~kg} \mathrm{~m}^{-1}$.

Figura 33. Comparação entre os resultados deste trabalho com outro numérico para o perfil de concentração, com $\Delta \mathrm{p}=1,010^{5} \mathrm{~Pa}, \mathrm{R}_{\mathrm{m}}=2,8 \quad 10^{9} \mathrm{~Pa} \mathrm{~s} \mathrm{~m}^{-1}, \mathrm{R}_{\mathrm{e}}=0,1 \quad 10^{9} \mathrm{~Pa} \mathrm{~s} \mathrm{~m}^{-1}$, $\phi=0,9610^{5} \mathrm{~s} \mathrm{~m}^{-1}, \mathrm{R}_{0}=1,510^{-2} \mathrm{~m}, \mu=10^{-3} \mathrm{~kg} \mathrm{~m}^{-1} \mathrm{~s}^{-1}, \rho=10^{3} \mathrm{~kg} \mathrm{~m}^{-1}, \mathrm{Sc}=14 \mathrm{e}$ $\mathrm{Re}_{\mathrm{w}}=0,52$.

Figura 34. Efeito do número de Reynolds sobre a espessura da camada limite de concentração em função da posição axial adimensional $\left(\mathrm{x} / \mathrm{R}_{0}\right)$, com $\mathrm{R}_{0}=3,010^{-3} \mathrm{~m}, \mathrm{Sc}=2500 \mathrm{e}$ $\operatorname{Re}_{\mathrm{w}}=0,005$.

Figura 35. Efeito do número de Reynolds sobre a concentração polarizada em função da posição axial adimensional $\left(\mathrm{x} / \mathrm{R}_{0}\right), \operatorname{com} \mathrm{R}_{0}=3,010^{-3} \mathrm{~m}, \mathrm{Re}_{\mathrm{w}}=0,005, \mathrm{Sc}=2500 \ldots 125$

Figura 36. Efeito do número de Reynolds de permeação sobre a espessura da camada limite de concentração em função da posição axial adimensional $\left(\mathrm{x} / \mathrm{R}_{0}\right)$, com $\mathrm{R}_{0}=3,010^{-}$ ${ }^{3} \mathrm{~m}, \mathrm{Re}=700, \mathrm{Sc}=2500$. 126

Figura 37. Efeito do número de Reynolds de permeação sobre a concentração polarizada em função da posição axial adimensional $\left(\mathrm{x} / \mathrm{R}_{0}\right)$, com $\mathrm{R}_{0}=3,010^{-3} \mathrm{~m}, \mathrm{Re}=700$, $\mathrm{Sc}=2500$. 126

Figura 38. Efeito do número de Schmidt sobre as espessuras das camadas limite de concentração em função da posição axial adimensional $\left(\mathrm{x} / \mathrm{R}_{0}\right)$, com $\mathrm{Re}=700$, $\mathrm{Re}_{\mathrm{w}}=0,005$ e $\mathrm{R}_{0}=3,010^{-3} \mathrm{~m}$.

Figura 39. Efeito do número de Schmidt sobre a concentração polarizada em função da posição axial adimensional $\left(\mathrm{x} / \mathrm{R}_{0}\right)$, com $\mathrm{R}_{0}=3,010^{-3} \mathrm{~m}, \mathrm{Re}=700, \mathrm{Re}_{\mathrm{w}}=0,005 \ldots \ldots 127$

Figura 40. Comparação entre os resultados da espessura da camada limite de concentração deste trabalho com a literatura, com $\mathrm{L}=0,5 \mathrm{~m}, \mathrm{Re}=500, \mathrm{Sc}=1550, \mathrm{Re}_{\mathrm{w}}=0,01 \mathrm{e}$ $\iota_{\max }=0,262$. 
Figura 41. Comparação entre os resultados da espessura da camada limite de concentração deste trabalho (Equação (97)) com a literatura, com $\mathrm{L}=0,3 \mathrm{~m}, \mathrm{Re}=700, \mathrm{Sc}=1100$,

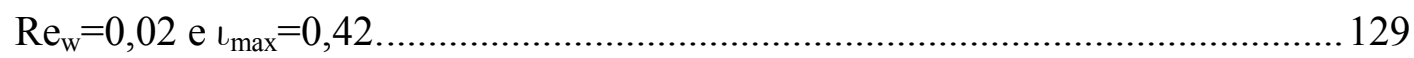

Figura 42. Representação da região entre dois tubos concêntricos...................................... 156 



\section{Lista de Tabelas}

Tabela 1. Intervalos aproximados do tamanho do poro de diferentes tipos de membranas sintéticas, comparado pelas dimensões de alguns componentes, separados pelo processo de filtração, bem como alguns exemplos. (adaptado de CHERYAN, 1998).

Tabela 2. Resumo do comportamento dos efeitos físicos envolvidos nos processos de filtração tangencial. 104

Tabela 3. (Equação Convectiva-Difusiva Parabólica) Análise da convergência da concentração $C$, na posição radial $R=0,98$ em função da coordenada axial $x / R_{0}$, com limite de tolerância igual a $10^{-3}, \mathrm{Sc}=2500, \mathrm{Re}_{\mathrm{w}}=0,01, \mathrm{Re}=900, \mathrm{R}_{0}=3,010^{-3} \mathrm{~m} \mathrm{e}$ $\varepsilon=0,01$. 106

Tabela 4. (Equação Convectiva-Difusiva Elíptica) Análise da convergência da concentração $\mathrm{C}$, na posição radial $\mathrm{R}=0,98$ em função da coordenada axial $\mathrm{x} / \mathrm{R}_{0}$, com limite de tolerância igual a $10^{-3}, \mathrm{Sc}=2500, \mathrm{Re}_{\mathrm{w}}=0,01, \mathrm{Re}=900, \mathrm{R}_{0}=3,010^{-3} \mathrm{~m}$ e $\varepsilon=0,01 \ldots .107$

Tabela 5. Resumo das comparações da concentração, na saída do tubo, $L / R_{0}=100$, entre o modelo elíptico e parabólico. Indicação da diferença percentual.

Tabela 6. Continuação do resumo das comparações da concentração, na saída do tubo, $\mathrm{L} / \mathrm{R}_{0}=100$, entre o modelo elíptico e parabólico. Indicação da diferença percentual.

Tabela 7. Resumo das comparações do adimensional de Sherwood, na saída do tubo, $\mathrm{L} / \mathrm{R}_{0}=100$, entre os modelos elíptico e parabólico. Representação da diferença percentual, onde o sinal negativo indica que o valor do modelo paróbolico é maior.

Tabela 8. Continuação do resumo das comparações do adimensional de Sherwood, na saída do tubo, $\mathrm{L} / \mathrm{R}_{0}=100$, entre os modelos elíptico e parabólico. Representação da diferença percentual, onde o sinal negativo indica que o valor do modelo 
parabólico é maior.

Tabela 9 . Valores de "Sh" provindos da solução do modelo em referência, via GITT, para determinação de $\mathrm{Sh}_{\text {aprox }}$, com $\mathrm{R}_{0}=3.010^{-3} \mathrm{~m}, 200 \leq \mathrm{Re} \leq 800,800 \leq \mathrm{Sc} \leq 2500$, $\mathrm{Re}_{\mathrm{w}}=0,005$ e $\varepsilon=0,01$. 164

Tabela 10. Quadro para análise estatística dos parâmetros “s” e $\alpha$. 165

Tabela 11. Valores de "Sh" provindos da solução do modelo em referência, via GITT, para determinação de $\mathrm{Sh}_{\text {aprox }}$, com $200 \leq \mathrm{Re} \leq 800,800 \leq \mathrm{Sc} \leq 2500,0,005 \leq \mathrm{Re}_{\mathrm{w}} \leq 0,014$ e $\mathrm{R}_{0}=3.010^{-3} \mathrm{~m}$. 166

Tabela 12 . Quadro para análise estatística dos parâmetros $\mathrm{w}, \zeta, \gamma, \lambda$ 166

Tabela 13. Resultados para a taxa assintótica e a taxa assintótica máxima, a partir dos limites inferiores e superiores dos intervalos para "Re", "Sc" e "Re $e_{\mathrm{w}}$ ", onde $\varepsilon=0,01 \ldots . .167$

Tabela 14 . Valores de "Sh" provindos da solução do modelo em referência, via GITT, para determinação de $\mathrm{Sh}_{\text {aprox, }}$ com $\mathrm{R}_{0}=3.010^{-3} \mathrm{~m}, 200 \leq \mathrm{Re} \leq 800,800 \leq \mathrm{Sc} \leq 2500$, $\mathrm{Re}_{\mathrm{w}}=0,005$ e $\varepsilon=0,01$. 168

Tabela 15 . Quadro para análise estatística dos parâmetros "s" e $\alpha$. 169

Tabela 16. Valores de "Sh" provindos da solução do modelo em referência, via GITT, para determinação de $\mathrm{Sh}_{\text {aprox }}$, com $200 \leq \mathrm{Re} \leq 800,800 \leq \mathrm{Sc} \leq 2500,0,005 \leq \mathrm{Re}_{\mathrm{w}} \leq 0,014$ e $\mathrm{R}_{0}=3,010^{-3} \mathrm{~m}$. 170

Tabela 17 . Quadro para análise estatística dos parâmetros $\mathrm{w}, \zeta, \gamma, \lambda$ 170

Tabela 18. Resultados para a taxa assintótica e a taxa assintótica máxima, a partir dos limites inferiores e superiores dos intervalos para "Re", "Sc" e "Re $e_{w}$ ", com $\varepsilon=0,01 \ldots . .171$

Tabela 19. Dados experimentais (PARIS et al., 2002).

Tabela 20. Resultados provindos da regressão linear, para a Equação (147) e Tabela 19. ... 174 


\section{Lista de Símbolos}

\begin{tabular}{|c|c|c|}
\hline Símbolo & Quantidade Física Escalar & Unidade \\
\hline $\mathrm{c}$ & Concentração & $\mathrm{kg} \mathrm{m}^{-3}$ \\
\hline $\mathrm{c}_{\mathrm{b}}$ & Concentração do alimentador central $\left(\mathrm{c}_{\mathrm{b}}=\mathrm{c}_{0}\right)$ & $\mathrm{kg} \mathrm{m}^{-3}$ \\
\hline $\mathrm{c}_{\mathrm{f}}$ & Concentração de filtrado ou solvente & $\mathrm{kg} \mathrm{m}^{-3}$ \\
\hline $\mathrm{c}_{\mathrm{w}}$ & Concentração na parede permeável & $\mathrm{kg} \mathrm{m}^{-3}$ \\
\hline $\mathrm{c}_{0}$ & Concentração de entrada & $\mathrm{kg} \mathrm{m}^{-3}$ \\
\hline $\mathrm{C}_{\mathrm{H}}$ & Potencial transformado para concentração (Equação (68)) & - \\
\hline$\overline{\mathrm{C}}_{\mathrm{i}}$ & Transformada Integral (Equação (67)) & - \\
\hline $\mathrm{D}$ & Difusividade mássica & $\mathrm{m}^{2} \mathrm{~s}^{-1}$ \\
\hline $\mathrm{F}$ & Filtro (Equação (110)) & - \\
\hline $\mathrm{J}$ & Fluxo transmembrana local & $\begin{array}{l}\mathrm{m} \mathrm{s}^{-1} \text { ou } \\
1 \mathrm{~h}^{-1} \mathrm{~m}^{-2}\end{array}$ \\
\hline $\mathrm{J}_{\lim }$ & Fluxo transmembrana (Equação (39)) & $\begin{array}{l}\mathrm{m} \mathrm{s}^{-1} \mathrm{ou} \\
1 \mathrm{~h}^{-1} \mathrm{~m}^{-2}\end{array}$ \\
\hline $\mathrm{J}_{\mathrm{m}}$ & Fluxo transmembrana médio & $\begin{array}{l}\mathrm{m} \mathrm{s}^{-1} \text { ou } \\
1 \mathrm{~h}^{-1} \mathrm{~m}^{-2}\end{array}$ \\
\hline $\mathrm{J}_{\mathrm{m}, \exp }$ & Fluxo transmembrana médio (experimental) & $\begin{array}{l}\mathrm{m} \mathrm{s}^{-1} \text { ou } \\
1 \mathrm{~h}^{-1} \mathrm{~m}^{-2}\end{array}$ \\
\hline $\mathrm{k}$ & Coeficiente de transferência de massa & $\mathrm{m} \mathrm{s}^{-1}$ \\
\hline $\mathrm{L}$ & Comprimento do tubo & $\mathrm{m}$ \\
\hline $\mathrm{N}$ & Ordem de truncamento & - \\
\hline $\mathrm{N}_{\mathrm{i}}$ & Integral de normalização (Equação (66)) & - \\
\hline $\mathrm{N}_{\mathrm{S}}$ & Fluxo de soluto dentro do poro (Equação (14)) & $\mathrm{m} \mathrm{s}^{-1}$ \\
\hline $\mathrm{p}$ & Pressão & $\mathrm{m}^{-1} \mathrm{~kg} \mathrm{~s}^{-2}$ \\
\hline$\Delta \mathrm{p}$ & Pressão transmembrana & $\mathrm{m}^{-1} \mathrm{~kg} \mathrm{~s}^{-2}$ \\
\hline$p_{\text {ext }}$ & Pressão Externa (permeado, solvente ou filtrado) & $\mathrm{m}^{-1} \mathrm{~kg} \mathrm{~s}^{-2}$ \\
\hline $\mathrm{p}_{0}$ & Pressão de entrada & $\mathrm{m}^{-1} \mathrm{~kg} \mathrm{~s}^{-2}$ \\
\hline $\mathrm{r}$ & Eixo radial (coordenadas cilíndricas) & $\mathrm{m}$ \\
\hline
\end{tabular}




\begin{tabular}{|c|c|c|}
\hline $\mathrm{R}_{\mathrm{e}}$ & Resistência do entupimento da membrana (fouling) & $\mathrm{m}^{-2} \mathrm{~kg} \mathrm{~s}^{-1}$ \\
\hline $\mathrm{R}_{\mathrm{g}}$ & $\begin{array}{l}\text { Resistência da concentração polarizada e da camada limite } \\
\text { de concentração }\end{array}$ & $\mathrm{m}^{-2} \mathrm{~kg} \mathrm{~s}^{-1}$ \\
\hline $\mathrm{R}_{\mathrm{m}}$ & Resistência da membrana & $\mathrm{m}^{-2} \mathrm{~kg} \mathrm{~s}^{-1}$ \\
\hline $\mathrm{R}_{\min }$ & Diâmetro do tubo concêntrico menor (Eqs. (59) até (61)) & $\mathrm{m}$ \\
\hline $\mathrm{R}_{0}$ & Raio do tubo & $\mathrm{m}$ \\
\hline $\mathrm{u}$ & Velocidade axial & $\mathrm{m} \mathrm{s}^{-1}$ \\
\hline $\mathrm{u}_{0}$ & Velocidade axial média na entrada & $\mathrm{ms}^{-1}$ \\
\hline $\mathrm{v}$ & Velocidade radial & $\mathrm{m} \mathrm{s}^{-1}$ \\
\hline $\mathrm{V}_{\mathrm{w}}$ & Velocidade radial de permeação na parede & $\mathrm{m} \mathrm{s}^{-1}$ \\
\hline $\mathrm{x}$ & Eixo axial, direção tangencial (coord. cilíndricas) & $\mathrm{m}$ \\
\hline $\mathrm{y}$ & Eixo axial, direção transmembrana (coord. retangulares) & $\mathrm{m}$ \\
\hline \multicolumn{3}{|c|}{ SÍMBOLOS GREGOS } \\
\hline$\beta_{\mathrm{i}}$ & Autovalores (Equação (63)) & - \\
\hline$\delta$ & Espessura da camada limite de concentração(Eq. (97)) & $\mathrm{m}$ \\
\hline$\delta_{\mathrm{m}}$ & Espessura da membrana & $\mathrm{m}$ \\
\hline$\delta_{\mathrm{ij}}$ & Símbolo de Kronecker & - \\
\hline$\varepsilon$ & Erro percentual máximo & - \\
\hline$\phi$ & Coeficiente de proporcionalidade (Equação (40)) & $\mathrm{m}^{-1} \mathrm{~s}$ \\
\hline$\phi_{\mathrm{c}}$ & Fluxo convectivo (Equação (12)) & $\mathrm{m} \mathrm{s}^{-1}$ \\
\hline$\phi_{\mathrm{d}}$ & Fluxo difusivo (Equação (13)) & $\mathrm{m} \mathrm{s}^{-1}$ \\
\hline 1 & Taxa assintótica & - \\
\hline$l_{\max }$ & Taxa assintótica máxima (Equação (103)) & - \\
\hline$\kappa$ & Permeabilidade hidráulica & $\mathrm{m}^{2} \mathrm{~kg}^{-1} \mathrm{~s}$ \\
\hline$\mu$ & Viscosidade dinâmica & $\mathrm{m}^{-1} \mathrm{~kg} \mathrm{~s}^{-1}$ \\
\hline$\pi_{\mathrm{w}}$ & Pressão na parede & $\mathrm{m}^{-1} \mathrm{~kg} \mathrm{~s}^{-2}$ \\
\hline$\pi_{\mathrm{p}}$ & Pressão do permeado (filtrado ou soluto) & $\mathrm{m}^{-1} \mathrm{~kg} \mathrm{~s}^{-2}$ \\
\hline$\Delta \pi$ & Diferença de pressão osmótica & $\mathrm{m}^{-1} \mathrm{~kg} \mathrm{~s}^{-2}$ \\
\hline$\rho$ & Densidade & $\mathrm{kg} \mathrm{\textrm {m } ^ { - 3 }}$ \\
\hline$\psi_{\mathrm{i}}$ & Autofunções (Equação (62)) & - \\
\hline$\tilde{\psi}_{\mathrm{i}}$ & Autofunções normalizadas (Equação (64)) & - \\
\hline$\xi$ & Adimensional de posição $\left(=\mathrm{L} / \mathrm{R}_{0}\right)$ & \\
\hline
\end{tabular}




\begin{tabular}{|c|c|c|}
\hline \multicolumn{3}{|c|}{ ADIMENSIONAIS } \\
\hline Símbolo & Descrição & Definição \\
\hline $\mathrm{C}$ & Concentração & $\left(=\mathrm{c} / \mathrm{c}_{0}\right)$ \\
\hline $\mathrm{C}_{\mathrm{w}}$ & Concentração de polarização & $\left(=\mathrm{c}_{\mathrm{w}} / \mathrm{c}_{0}\right)$ \\
\hline $\mathrm{P}$ & Pressão & $\left(=\mathrm{p}-\mathrm{p}_{0} / \rho \mathrm{u}_{0}^{2}\right)$ \\
\hline $\mathrm{Pe}$ & Número de Peclet $(=$ Re Sc) & $\left(=\mathrm{u}_{0} \mathrm{R}_{0} / \mathrm{D}\right)$ \\
\hline $\operatorname{Re}$ & Número de Reynolds axial & $\left(=\rho \mathrm{u}_{0} \mathrm{R}_{0} / \mu\right)$ \\
\hline $\operatorname{Re}_{\mathrm{w}}$ & Número de Reynolds na parede permeável & $\left(=\rho \mathrm{v}_{\mathrm{w}} \mathrm{R}_{0} / \mu\right)$ \\
\hline $\mathrm{Sc}$ & Número de Schmidt & $(=\mu / \rho D)$ \\
\hline Sh & Número de Sherwood & $\left(=\mathrm{k} \mathrm{R}_{0} / \mathrm{D}\right)$ \\
\hline $\mathrm{U}$ & Velocidade axial & $\left(=\mathrm{u} / \mathrm{u}_{0}\right)$ \\
\hline $\mathrm{V}$ & Velocidade radial & $\left(=\mathrm{v} / \mathrm{u}_{0}\right)$ \\
\hline $\mathrm{V}_{\mathrm{w}}$ & Velocidade radial na parede & $\left(=\mathrm{v}_{\mathrm{w}} / \mathrm{u}_{0}\right)$ \\
\hline $\mathrm{R}$ & Eixo radial (coord. cilíndricas) & $\left(=\mathrm{r} / \mathrm{R}_{0}\right)$ \\
\hline $\mathrm{X}$ & Eixo longitudinal (coord. cilíndricas) & $\left(=\mathrm{x} / \mathrm{R}_{0}\right)$ \\
\hline Y & Eixo transversal (coord. retangulares) & $(=\mathrm{y} / \mathrm{L})$ \\
\hline \multicolumn{3}{|c|}{ ÍNDICES SUBSCRITOS } \\
\hline 0 & \multicolumn{2}{|c|}{ Condição de entrada do tubo e ordem das funções de Bessel } \\
\hline $\mathrm{b}$ & \multicolumn{2}{|l|}{ Escoamento central } \\
\hline $\mathrm{e}$ & \multicolumn{2}{|l|}{ Entupimento } \\
\hline $\exp$ & \multicolumn{2}{|l|}{ Experimento } \\
\hline ext & \multicolumn{2}{|l|}{ Exterior } \\
\hline $\mathrm{f}$ & \multicolumn{2}{|l|}{ Filtrado } \\
\hline $\mathrm{g}$ & \multicolumn{2}{|l|}{ Concentração polarizada } \\
\hline $\mathrm{H}$ & \multicolumn{2}{|l|}{ Homogeneo } \\
\hline $\lim$ & \multicolumn{2}{|l|}{ Valor crítico } \\
\hline $\mathrm{m}$ & \multicolumn{2}{|l|}{ Valor médio e membrana } \\
\hline $\max$ & \multicolumn{2}{|l|}{ Máximo } \\
\hline $\min$ & \multicolumn{2}{|l|}{ Mínimo } \\
\hline $\mathrm{i}, \mathrm{j}$ & \multicolumn{2}{|l|}{ Ordem do problema de autovalor } \\
\hline $\mathrm{p}$ & \multicolumn{2}{|l|}{ Especificação de permeado } \\
\hline $\mathrm{w}$ & \multicolumn{2}{|c|}{ Condição na parede interna do tubo ou membrana } \\
\hline
\end{tabular}





\section{Glossário}

GITT (GENERALIZED INTEGRAL TRANSFORM TECHNIQUE): sigla conhecida internacionalmente que significa Técnica da Transformada Integral Generalizada.

CITT (CLASSICAL INTEGRAL TRANSFORM TECHNIQUE): sigla que significa Técnica da Transformada Integral Clássica.

MF: Microfiltração.

UF: Ultrafiltração

NF: Nanofiltração 



\section{Lista de Apêndices}

Apêndice 1: Perfil de concentração para um escoamento laminar completamente

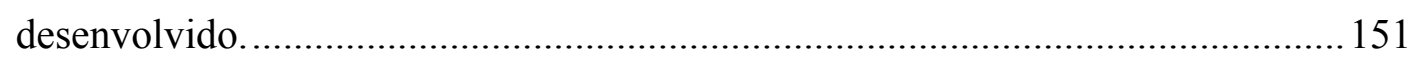

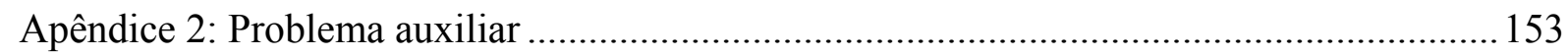

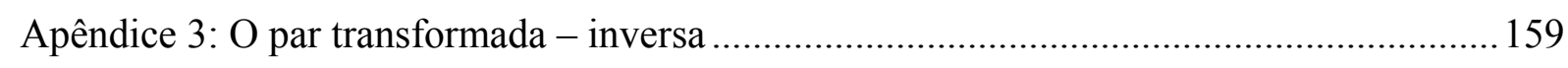

Apêndice 4: Avaliação dos Parâmetros da Correlação de Gilland-Sherwood. ....................... 163

Apêndice 5: Resultados experimentais - Paris et al. (2002). .............................................. 173

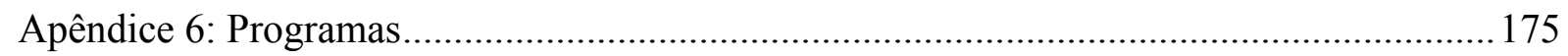





\section{Sumário}

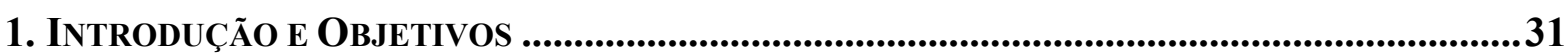

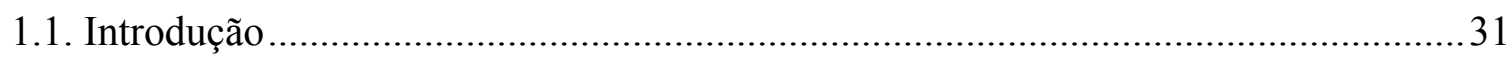

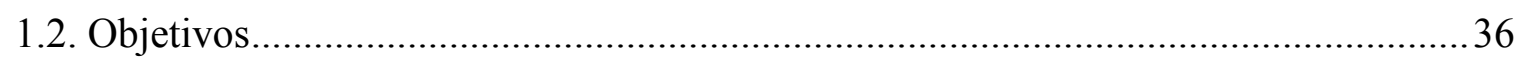

2. REVISÃO DA LITERATURA ...............................................................................................39

2.1. Mecanismo de transferência de massa na filtração tangencial .....................................39

2.1.1. Processos de Filtração e Filtração Tangencial..............................................39

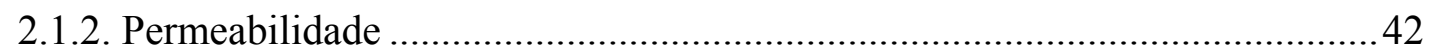

2.1.3. Transporte de soluto e solvente na membrana................................................ 43

2.1.4. Camada Limite de Concentração e Concentração Polarizada ...........................45

2.1.5. Conservação da Massa na Superfície Permeável ............................................. 48

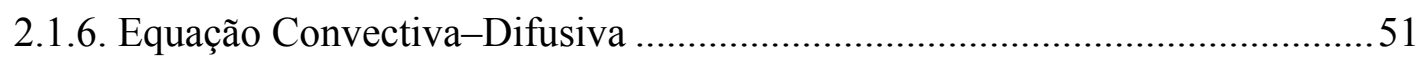

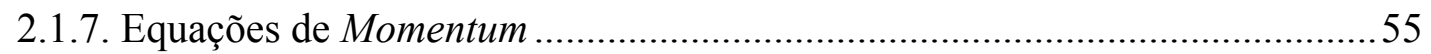

2.1.8. Entupimento da Membrana (Fouling) .........................................................59

2.1.9. Modelo da Camada de Gel Polarizado e Modelo da Resistência em Série....61

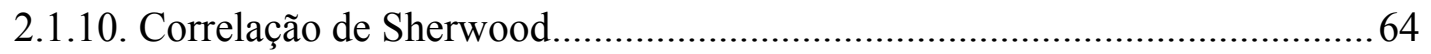

2.2. Modelagem dos Processos de Filtração Tangencial ....................................................66

2.3. Técnica de Transformada Integral Generalizada (GITT) ………………………......72

3. FoRMULAÇ̃̃o MATEMÁTICA E RESOLUÇões VIA GITT .....................................................81

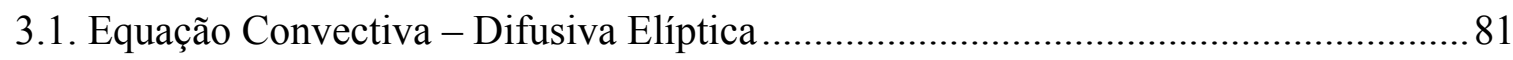

3.1.1. Formulação e Modelagem Matemática ………………………………............. 81

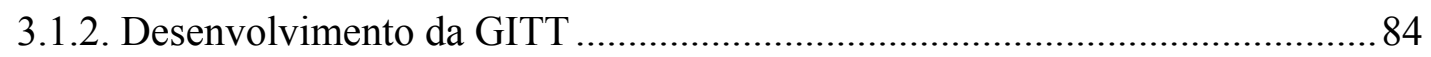

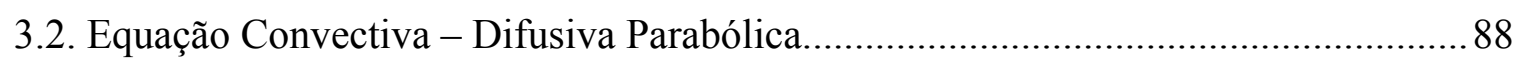

3.2.1. Formulação e Modelagem Matemática ......................................................... 88

3.2.2. Desenvolvimento da GITT ……………………………........................... 89

3.3. Formulação da Espessura da Camada Limite de Concentração e Concentração

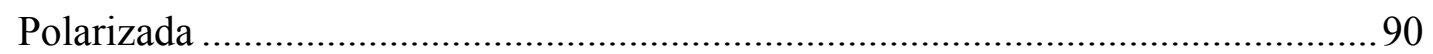

3.3.1. Formulação Matemática ………………………………………………....... 90

3.3.2. Metodologia para Determinação da Taxa Assintótica.......................................94 


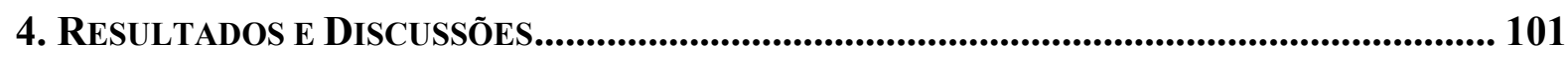

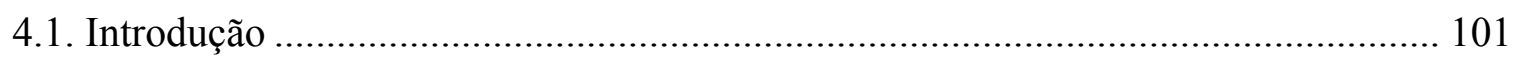

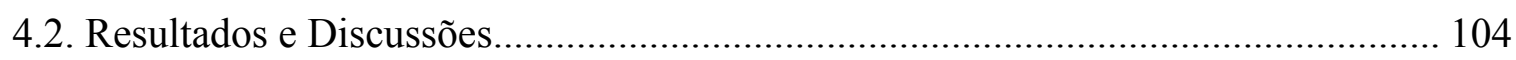

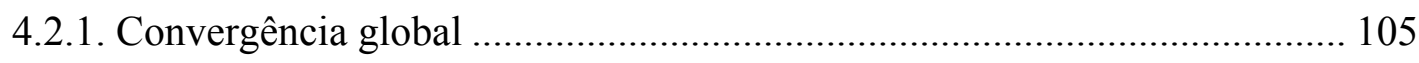

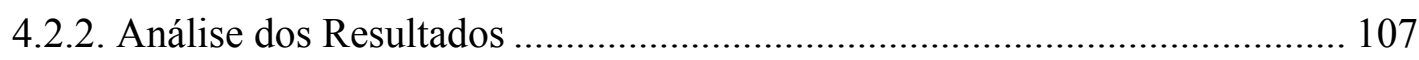

4.2.3. Comparações do Adimensional de Sherwood com Outros Resultados da

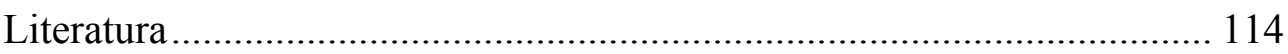

4.2.4. Comparações do Fluxo Transmembrana e do Perfil de Concentração com Outros Resultados da Literatura.............................................................. 119

4.2.5. Análise da Espessura da Camada Limite de Concentração ........................ 123

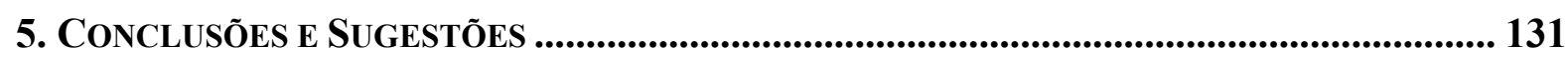

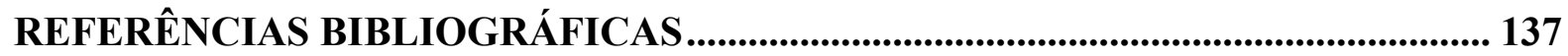




\section{Introdução e Objetivos}

\subsection{INTRODUÇÃo}

Os fundamentos do processo de filtração tangencial estão associados ao escoamento confinado numa superfície permeável, em geral num tubo permeável, ou membrana tubular. A necessidade de estender à área de filtração e obter volumes satisfatórios de filtrado ou permeado, exige a confecção de módulos, ou seja, feixes de tubos dentro de um casco, análogo a um trocador de calor de casco e tubos.

Um dos problemas existentes nos projetos de módulos com membranas em processos de filtração tangencial é a caracterização dos mecanismos da transferência de massa do elemento filtrante. A seletividade intrínseca da superfície permeável, combinada com a vazão transmembrana, somente será adequada se a taxa de transferência de massa do soluto na solução for suficientemente grande para evitar a intensificação de uma camada limite de concentração de retentado de soluto na superfície permeável. Esta camada é usualmente denominada de concentração polarizada (ou camada de polarização) e é, freqüentemente, um fator dominante no controle do desempenho de processos de filtração. Para obtermos altas taxas de transferência de massa, necessita-se de altas velocidades tangenciais nas vizinhanças da superfície da membrana. Estas altas taxas de velocidades tangenciais são obtidas na 
condição de escoamento turbulento. Contudo, devido à utilização de módulos de membranas capilares, pode-se obter escoamento laminar com fluxo transmembrana satisfatório, o qual sofre influência dominante da camada de polarização e velocidade tangencial.

O escoamento associado ao processo de filtração tangencial é, essencialmente, um problema convectivo com crescimento simultâneo das camadas limites de velocidade e concentração. A investigação na aplicação de técnicas analítico-numéricas na modelagem do escoamento num tubo permeável é relevante, uma vez que visam estimativas de fluxos de filtração de um processo cada vez mais aplicado em diversas áreas da engenharia.

Na solução dos modelos associados aos processos de filtração tangencial, utilizaremos a técnica de transformada integral generalizada, conhecida internacionalmente por GITT, Generalized Integral Transform Technique, sobre a qual seguem alguns breves comentários.

Na segunda metade do século XX, uma grande quantidade de problemas do tipo difusivo-convectivo tem sido resolvida analiticamente mediante o uso das transformadas integrais. Este acúmulo de experiências foi sistematicamente registrado por Mikhailov e Özisik, (1984, apud PEREIRA, 2000) para sete classes de problemas.

A dificuldade da aplicação da técnica de transformada integral clássica reside em que um problema de autovalor apropriado não pode transformar completamente cada um dos termos de uma equação diferencial parcial, obtendo-se em tal caso um sistema diferencial ordinário acoplado, muitas vezes mostrando características de rigidez numérica (stiff system). Uma nova concepção na solução de problemas difusivos-convectivos foi proposta por Özisik e Murray (1974, apud PEREIRA, 2000), e posteriormente sistematizada e amplamente difundida por Cotta (1993) e Cotta e Mikhailov (1997). Neste novo enfoque, concebe-se uma solução híbrida numérico-analítica, onde a parte analítica consiste na transformada integral do 
problema, generalizando o conceito de transformada integral clássica, pelo que se obtém um sistema diferencial ordinário acoplado, produto da não transformação de todos os termos da equação diferencial. A tarefa numérica resume-se à resolução do sistema diferencial ordinário da transformada resultante.

Este método híbrido explora o amplo alcance que se tem na atualidade para solução numérica, com tolerância prescrita, de sistemas diferenciais ordinários. Este fato, conjuntamente com a característica analítica do método, oferece o controle sobre o erro global na solução do problema e faz com que a GITT seja uma ferramenta poderosa na obtenção de resultados de comparação ou benchmark. O método é também muito útil para obtenção de resultados de engenharia, pelo pequeno tempo de processamento na obtenção de resultados convergidos numa escala gráfica. Um aspecto interessante da GITT é a relativa facilidade de implementação computacional e isto torna o método atrativo na geração de códigos (PEREIRA, 2000).

O desenvolvimento deste trabalho segue os seguintes passos: (1) modelagem do escoamento e fluido utilizando a equação de conservação das espécies químicas, cuja condição de contorno na superfície porosa é dinâmica, sendo necessária a determinação de uma expressão matemática para a concentração na parede e a espessura da camada limite de concentração, (2) estabelecer uma metodologia para determinação da taxa assintótica, a qual ocorre na expressão da camada limite de concentração.

A partir da equação de balanço de massa na parede, obtivemos uma expressão matemática para a concentração na parede permeável e esta depende, entre outros parâmetros, da espessura da camada limite de concentração polarizada. A formulação para a concentração na parede deve ser bem determinada, ou seja, uma função contínua e diferenciável para todo intervalo relacionado às dimensões do tubo. 
$\mathrm{Na}$ literatura não encontramos uma formulação matemática geral para a espessura da camada limite de concentração polarizada que pudéssemos utilizar neste trabalho. Desta forma, construímos uma expressão para a mesma, a qual preserva a continuidade e diferenciabilidade da função de concentração na parede permeável. $\mathrm{Na}$ formulação matemática para a espessura da camada limite de concentração polarizada, há a dependência dos parâmetros adimensionais: Reynolds, Reynolds de permeação, Schmidt e uma taxa assintótica. Esta última nos trouxe um problema adicional, que foi resolvido com o desenvolvimento de uma metodologia para sua determinação. Nesta metodologia utilizamos, além da GITT, a suposição provinda da teoria do filme clássica, ou seja, a igualdade entre um fluxo transmembrana médio (modelo do gel polarizado) e a velocidade de permeação. Isto é possível, pois a velocidade de permeação é assumida como sendo uniforme e o resultado do fluxo transmembrana médio é obtido, via GITT. De fato, o fluxo transmembrana médio uniforme é provindo do fluxo transmembrana local, e este último é definido como proporcional ao coeficiente de transferência de massa na superfície permeável. A obtenção deste coeficiente é possível depois que determinamos o gradiente de concentração na parede permeável e este resultado é simulado pela GITT.

A metodologia para determinação da taxa assintótica inicia-se com uma estimativa inicial para a taxa assintótica que deve ser sempre menor que o valor da taxa assintótica máxima. Esta taxa máxima regula a coerência teórica e física da concentração na parede permeável. Para sabermos se o fluxo transmembrana médio e a velocidade de permeação na parede permeável são valores aproximados, construímos um intervalo centrado na velocidade de permeação e os extremos com proporcionalidade de $1 \%$ desta mesma velocidade de permeação. Assim, quando o fluxo transmembrana médio pertencer a este intervalo, determinamos a taxa assintótica, onde os parâmetros adimensionais: Reynolds, Reynolds de permeação e Schmidt são fixos. A partir desta taxa assintótica, obtemos a espessura da 
camada limite de concentração e, portanto, a concentração na parede, ficando bem determinada a condição de contorno na parede permeável.

Para a comparação dos resultados da transferência de massa na superfície permeável, construímos a correlação de Sherwood-Gilland, estabelecida para certos intervalos dos adimensionais de Reynolds, Reynolds de permeação e Schmidt. Esta correlação, definida para os intervalos dos adimensionais, nos garante a independência dos resultados da simulação da modelagem.

No Capítulo 2, temos a revisão da literatura onde explanamos sobre os mecanismos de transferência de massa na filtração tangencial, a modelagem dos processos de filtração tangencial e a GITT. Na seção sobre mecanismos de transferência de massa na filtração tangencial expusemos as teorias: permeabilidade, transporte de soluto e solvente na membrana, camada limite de concentração e concentração polarizada, conservação da massa na superfície permeável, equações de momentum, entupimento da membrana, modelos da camada de gel polarizado e da resistência em série e, finalmente, correlação de Sherwood.

No Capítulo 3 modelamos o escoamento em regime laminar e permanente, de fluido incompressível, newtoniano, confinado em tubos com superfícies permeáveis. Consideramos o fluido com uma mistura ideal para uma única espécie química; o modelo é especificado pelas equações convectiva-difusiva elíptica e convectiva-difusiva parabólica, onde os perfis de velocidade envolvidos são estabelecidos pela literatura (YUAN, FINKELSTEIN e BROOKLYN, 1956). Neste capítulo resolvemos, via GITT, os problemas modelados a partir da equação convectiva-difusiva elíptica e parabólica. Além disso, encontramos a formulação matemática para a espessura da camada limite de concentração.

$\mathrm{Na}$ introdução do Capítulo 4 temos uma análise do comportamento dos efeitos físicos 
envolvidos nos processos de filtração tangencial, bem como o estabelecimento de intervalos para os adimensionais relacionados a estes processos. Nos resultados e discussões a convergência global foi estabelecida para determinadas ordens de truncamento. Analisamos o comportamento do perfil de concentração e do adimensional de Sherwood, os quais se relacionam aos modelos elípticos e parabólicos. Foram realizadas comparações do fluxo transmembrana local e médio, o adimensional de Sherwood com resultados da literatura. Validamos a expressão matemática para a espessura da camada limite de concentração com resultados da literatura.

No Capítulo 5, temos as conclusões sobre o comportamento do resultado da simulação do perfil de concentração, adimensional de Sherwood e fluxo transmembrana relativos a cada uma das equações convectivas-difusivas modeladas no Capitulo 3. Apresentamos também algumas sugestões para futuros desenvolvimentos.

\subsection{OBJETIVOS}

O objetivo geral desta tese de doutorado é a modelagem e a solução, via GITT, do escoamento laminar incompressível, newtoniano e permanente em tubos permeáveis aplicada ao processo de filtração tangencial.

Os objetivos específicos são os seguintes:

i. Solucionar via GITT a equação de conservação das espécies químicas (equação convectiva-difusiva parabólica e elíptica), assumindo o escoamento laminar e permanente de um fluido incompressível e newtoniano em tubos permeáveis, onde as velocidades axial e radial são fornecidas pela literatura.

ii. Propor, para diferentes parâmetros que representam o escoamento e o fluido, uma expressão matemática para a espessura da camada limite de concentração; 
iii. Estabelecer, para diferentes parâmetros que caracterizam o escoamento e o fluido, uma metodologia para determinação da taxa assintótica;

iv. Determinar a correlação de Sherwood, a partir de simulações via GITT do modelo parabólico e elíptico e compará-los com outros resultados da literatura;

v. A partir de variações da equação convectiva-difusiva (parabólica e elíptica), analisar os efeitos dos termos convectivos e difusivos não lineares sobre os perfis de concentração e o adimensional de Sherwood.

Pretende-se, assim, contribuir com o desenvolvimento de modelagens e simulações dos processos de filtração tangencial, através de uma técnica híbrida analítico-numérica versátil, que proporciona a determinação de perfis de concentração, da espessura da camada limite de concentração, do adimensional de Sherwood e a verificação de estudos realizados por técnicas puramente numéricas para parâmetros que descrevem o problema físico. 



\section{Revisão da Literatura}

Em constantes pesquisas em sites específicos de busca (por exemplo, SCIRUS, 2007) ${ }^{\dagger}$, não constatamos a existência de trabalhos vinculados aos processos de filtração tangencial, cuja solução das equações que regem estes processos foi investigada pela GITT. Diante desta situação, os trabalhos discutidos nesta seção não serão tratados simultaneamente pela filtração tangencial e GITT, porém outros trabalhos de modelagem serão comentados, devido à sua relevância na abordagem do processo.

\subsection{MECANiSMO DE TRANSFERÊNCIA DE MASSA NA FILTRAÇ̃̃o TANGENCIAL}

Nos itens seguintes temos definições, características e elementos importantes para o desenvolvimento teórico dos processos de separação por membrana. Embora os modelos utilizados por este trabalho estejam em coordenadas cilíndricas, em alguns pontos desta seção, utilizamos as coordenadas retangulares, devido à importância no entendimento da teoria em estudo.

\subsubsection{Processos de Filtração e FiltraÇão Tangencial}

Filtração é definida como sendo a separação de dois ou mais componentes de um fluido

\footnotetext{
${ }^{\dagger}$ Algumas palavras chaves que combinamos com Integral Transform: Mathematical Modeling; Crossflow; Mass Transfer, Microfiltration, Ultrafiltration.
} 
escoando num meio material poroso, com características físicas que permitem a seleção dos componentes, principalmente, na diferença de tamanho de poros. Normalmente, separam-se partículas sólidas num fluxo líquido ou gasoso. Considera-se uma membrana como sendo uma barreira seletiva sintética, usada em processos industriais e em escalas laboratoriais. $\mathrm{O}$ processo de filtração por membrana inclui a separação de solutos dissolvidos em correntes líquidas e a separação de misturas em correntes gasosas (CHERYAN, 1998).

Tabela 1. Intervalos aproximados do tamanho do poro de diferentes tipos de membranas sintéticas, comparado pelas dimensões de alguns componentes, separados pelo processo de filtração, bem como alguns exemplos.

(adaptado de CHERYAN, 1998).

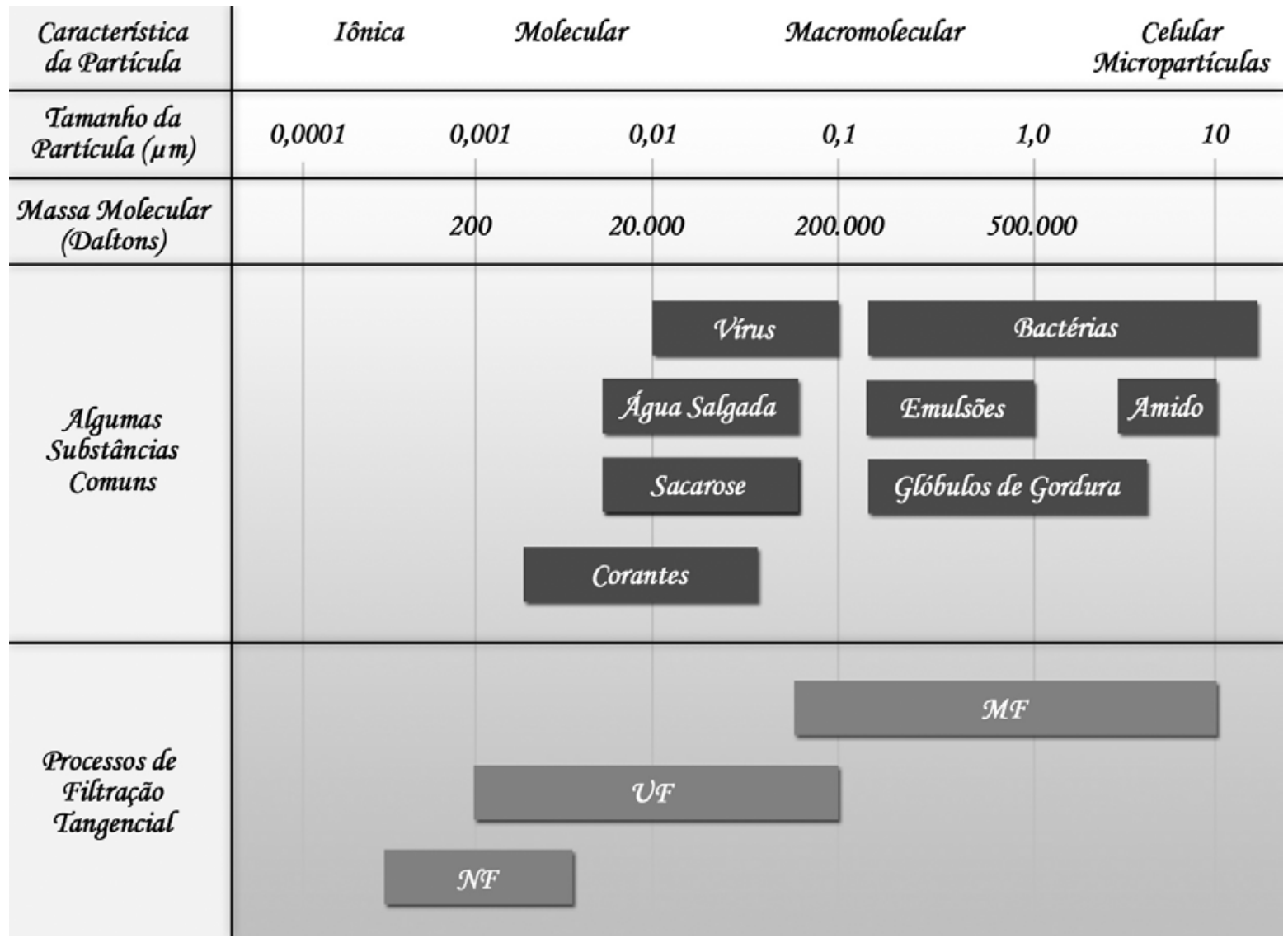

Nos processos de filtração tangencial, cuja força motriz é a pressão submetida numa superfície permeável, o objetivo é separar partículas finas em suspensão, microorganismos e emulsões. As membranas utilizadas possuem uma estrutura de microporos, separando as partículas de tamanhos que variam de $0,2 \mu \mathrm{m}$ até $5 \mu \mathrm{m}$, para microfiltração, MF, de 
$0,001 \mu \mathrm{m}$ até $0,2 \mu \mathrm{m}$ para ultrafiltração, UF, e entre $0,0005 \mu \mathrm{m}$ e $0,005 \mu \mathrm{m}$, para nanofiltração, NF. Em ultrafiltração a separação está baseada no efeito de peneira, portanto, o efeito de separação é limitado principalmente na superfície exterior da membrana (RIPPERGER e ALTMANN, 2002). Os intervalos aproximados do tamanho do poro de diferentes tipos de membranas sintéticas podem ser vistos na Tabela 1.

Além do processo citado, as membranas podem ser classificadas de várias outras formas, tais como, estrutura da membrana (porosos ou não-porosos), aplicação da membrana (separação de fase gasosa, gás-líquido, líquido-líquido), mecanismo de ação da membrana (adsorção-difusivo, troca iônica, osmótica ou membrana não-seletiva (inerte)) (CHERYAN, 1998).

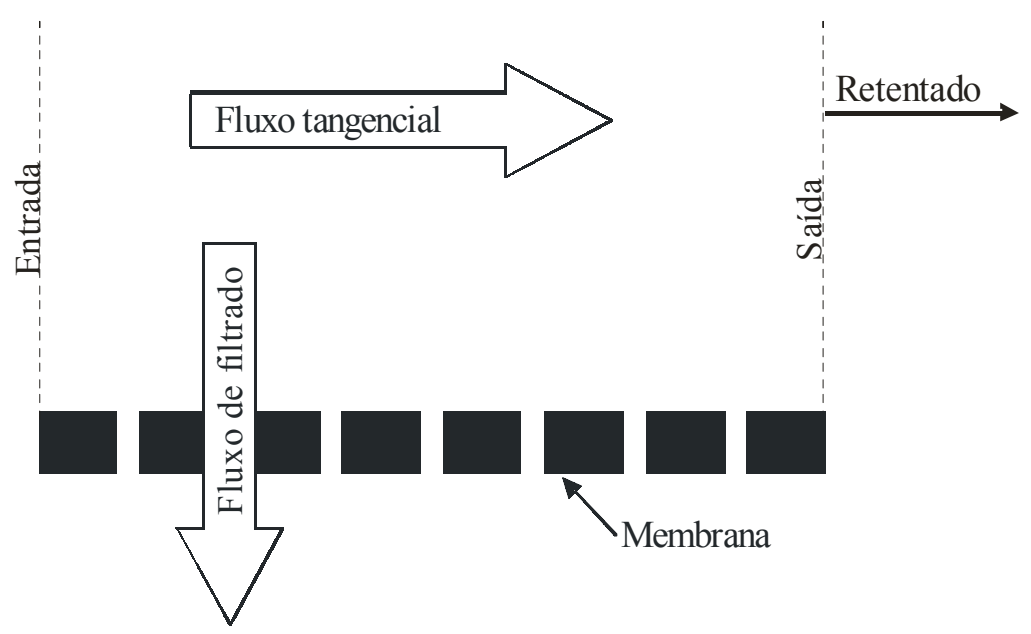

Figura 1. Princípio da filtração tangencial, onde temos o fluxo de filtrado (ou fluxo transmembrana), o fluxo tangencial e do retentado (Adaptado de RIPPERGER e ALTMANN, 2002).

Existem dois tipos de processos de filtração: a tangencial e a perpendicular (filtro prensa), os quais são de grande utilização em processos industriais. A filtração tangencial ocorre tangencialmente à membrana e, neste processo, é mantida a mistura fluida circulando paralelamente a esta superfície permeável e sujeita às diferenças de pressões transmembrana. $\mathrm{Na}$ filtração perpendicular, a mistura fluida ocorre perpendicularmente à membrana e neste processo as partículas se acumulam na superfície permeável, formando uma camada de 
filtrado sólido. $\mathrm{Na}$ filtração tangencial, os processos de transferência são extremamente complexos, requerendo um conhecimento, em particular, das características físicas e químicas da solução de alimentação, da vazão, do regime de escoamento e de propriedades da membrana permeável e camada de polarização (QUEIROZ, 2004). A Figura 1 mostra o principio da filtração tangencial, bem como a indicação do retentado (aquela porção da solução que não fica retida sobre membrana).

\subsubsection{Permeabilidade}

Um importante indicador da funcionalidade da membrana para microfiltração/ultrafiltração é a permeabilidade. A relação entre fluxo transmembrana, J, e pressão transmembrana, $\Delta \mathrm{p}$, é descrita pela lei de Darcy (ZEMAN e ZYDNEY, 1996):

$$
\mathrm{J}=\kappa \Delta \mathrm{p}
$$

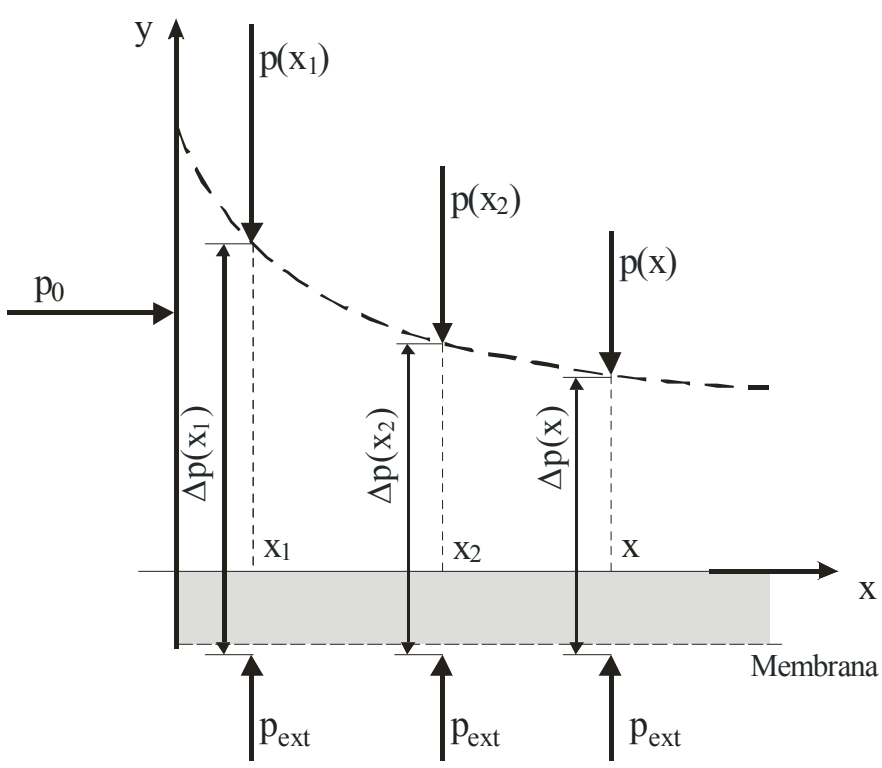

Figura 2. Representação esquemática da pressão transmembrana, $\Delta \mathrm{p}$, da pressão de entrada, $\mathrm{p}_{0}$, da pressão do permeado (ou exterior), $\mathrm{p}_{\mathrm{ext}}$, e a pressão local $\mathrm{p}(\mathrm{x})$.

A Figura 2 mostra, esquematicamente, o comportamento da pressão transmembrana local, a qual envolve as pressões de entrada e do permeado, bem como a pressão local do escoamento. 
Nos processos de filtração tangencial a força motriz é a pressão transmembrana. A Figura 2 mostra a pressão transmembrana local, $\Delta \mathrm{p}(\mathrm{x})$, para cada posição axial, $\mathrm{x}$, da membrana, definida por: $\Delta \mathrm{p}(\mathrm{x})=\mathrm{p}(\mathrm{x})-\mathrm{p}_{\mathrm{ext}}$.

A resistência hidráulica da membrana mede o grau de impedimento, pelo próprio material da membrana ou o entupimento (fouling), do fluxo de água através da membrana (UNITED STATES ENVIRONMENTAL PROTECTION AGENCY, 2003), a qual é proporcional ao inverso da permeabilidade hidráulica da membrana, isto é:

$$
\mathrm{R}_{\mathrm{m}}=\frac{1}{\kappa}
$$

\subsubsection{TRANSPORTE DE SOLUTO E SOLVENTE NA MEMBRANA}

A morfologia dos poros de uma membrana é extremamente complexa. Examinando o comportamento de um modelo composto de uma matriz de poros cilíndricos e paralelos, é possível obter considerável compreensão sobre as características das membranas de ultrafiltração e microfiltração. Para este caso, a velocidade de filtração (ou velocidade na parede), $\mathrm{v}_{\mathrm{w}}$, é dada pela lei de Poiseuille:

$$
\mathrm{v}_{\mathrm{w}}=\frac{\mathrm{r}_{\mathrm{po}}^{2} \Delta \mathrm{p} 10^{-5}}{288 \mu \delta_{\mathrm{m}}}
$$

com "r $\mathrm{r}_{\mathrm{po}}$ " sendo o raio do poro (ZEMAN e ZYDNEY, 1996). Esta lei é somente válida na ausência de qualquer pressão osmótica ou efeito de rejeição de soluto.

A Figura 3 mostra esquematicamente um poro cilíndrico. A concentração na parede, $\mathrm{c}_{\mathrm{w}}$, é a concentração na camada adjacente à superfície da membrana, a qual será vista com mais detalhes no Item 2.1.4. A concentração de filtrado (ou permeado), $\mathrm{c}_{\mathrm{f}}$, é a concentração existente no fluxo de filtrado através da membrana. 


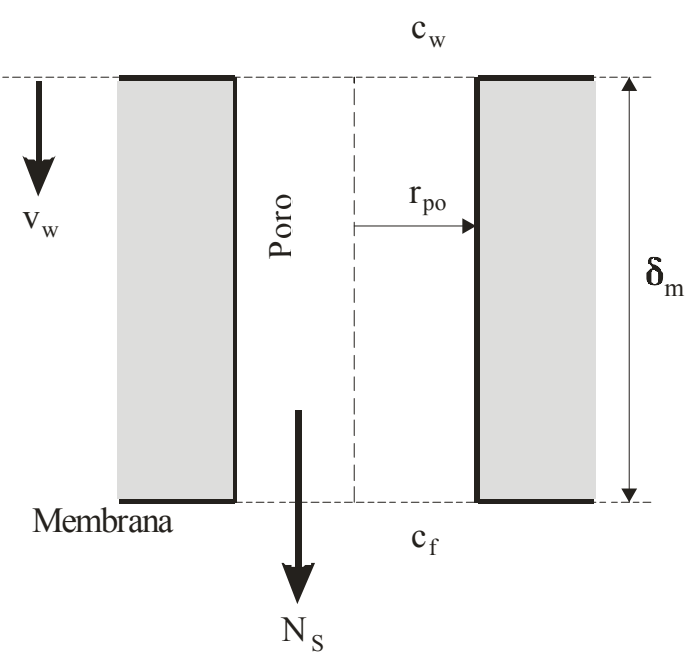

Figura 3. Representação esquemática de um poro cilíndrico, bem como as indicações da concentração na parede, $\mathrm{c}_{\mathrm{w}}$, a concentração de filtrado, $\mathrm{c}_{\mathrm{f}}$, o raio do poro, $\mathrm{r}_{\mathrm{po}}$, a espessura da membrana, $\delta_{\mathrm{m}}$, a velocidade na parede, $\mathrm{v}_{\mathrm{w}}, \mathrm{e}$ o fluxo de soluto dentro do poro, $\mathrm{N}_{\mathrm{S}}$.

Em muitos dispositivos de filtração, a concentração de soluto no fluxo de filtrado é determinada pelos fluxos de soluto e solvente através da membrana. Com isto, o balanço de massa do fluxo de filtrado pode ser escrito como (ZEMAN e ZYDNEY, 1996): $\mathrm{c}_{\mathrm{f}}=\mathrm{N}_{\mathrm{S}} / \mathrm{v}_{\mathrm{w}}$.

O gradiente de concentração através da membrana pode causar uma diferença na pressão osmótica sobre os dois lados da membrana. Quando o soluto é impedido de difundir através de uma membrana permeável ao solvente, o solvente tenderá a difundir de áreas de alta concentração de água (e baixa concentração de soluto) para região de baixa concentração de solvente (e alta concentração de soluto). Isto resulta em uma força chamada diferença de pressão osmótica, $\Delta \pi=\pi_{\mathrm{w}}-\pi_{\mathrm{p}}$. A diferença de pressão osmótica gera uma contribuição para o fluxo transmembrana total, dada pela Equação (1) (ZEMAN e ZYDNEY, 1996), isto é,

$$
\mathrm{J}=\kappa \Delta \mathrm{p}+\mathrm{A}_{\mathrm{p}} \Delta \pi
$$

com $A_{p}$ sendo o coeficiente fenomenológico que descreve a magnitude do fluxo osmótico.

O coeficiente de reflexão osmótica, ou coeficiente de Staverman, é dado por: 


$$
\sigma_{0}=-\frac{A_{p}}{\kappa}=\left.\frac{\Delta p}{\Delta \pi}\right|_{J=0}
$$

A expressão $\left.\frac{\Delta \mathrm{p}}{\Delta \pi}\right|_{\mathrm{J}=0}$ é usada na determinação experimental de $\sigma_{0}$. Para membranas que rejeitam completamente o soluto, tem-se $\sigma_{0}=1$, e para membranas que não rejeitam completamente o soluto, tem-se $\sigma_{0}=0$. No caso onde $\sigma_{0}=1$ temos que $\Delta \mathrm{p}=\Delta \pi$ e $\mathrm{J}=0$ e, neste caso, os potenciais químicos do solvente sobre os dois lados da membrana são iguais, de acordo com a condição termodinâmica através de um limite semipermeável. Assim, a Equação (4) pode ser escrita na forma (ZEMAN e ZYDNEY, 1996):

$$
\mathrm{J}=\kappa\left(\Delta \mathrm{p}-\sigma_{0} \Delta \pi\right)
$$

\subsubsection{Camada Limite de ConcentraÇão e ConcentraÇão Polarizada}

Consideremos um escoamento de um fluido sobre uma superfície plana impermeável. Quando partículas do fluido entram em contato com a superfície, elas passam a ter velocidade nula. Estas partículas atuam então na desaceleração do movimento das partículas da camada de fluido adjacente, que, por sua vez, atuam na desaceleração do movimento das partículas da próxima camada e assim sucessivamente, até uma distância da superfície, $y=\delta_{v}$, onde o efeito de retardamento se torna desprezível (INCROPERA e De WITT, 1998). A região desenvolvida entre a superfície da membrana, $y=0$, até uma distância da superfície, $y=\delta_{v}$, é conhecida por camada limite de velocidade, cuja espessura é $\delta_{\mathrm{v}}$.

Analogamente à forma que a camada limite de velocidade determina o atrito por convecção numa superfície plana impermeável, a camada limite de concentração determina a transferência de massa por convecção. Se uma mistura de espécies químicas, por exemplo, A e B, escoa sobre uma superfície e a concentração da espécie A na superfície é diferente 
daquela no escoamento central, irá se desenvolver uma camada limite de concentração. Ela é uma região do fluido, de espessura $\delta$, onde existem gradientes de concentração (INCROPERA e De WITT, 1998).

Nos processos de filtração tangencial onde estão presentes as superfícies permeáveis, as partículas são levadas da corrente livre para a superfície da membrana através do transporte convectivo, e uma parte do solvente é removido do fluido. Isto resulta em um aumento na concentração local de soluto rejeitado na superfície da membrana, se comparada à concentração de entrada, independentemente se os solutos são parcialmente ou completamente rejeitados pela membrana. No fluido (soluto e solvente) do escoamento central, ou seja, fora da camada limite, assume-se geralmente que o perfil de concentração $\left(c_{b}\right)$ é essencialmente uniforme na direção perpendicular à superfície da membrana (CHERYAN, 1998).

Consideremos um fluxo, através de uma superfície permeável, de um fluido (soluto e solvente). Esta mistura estabelece junto à superfície uma camada de estagnação associada à camada limite de concentração. Isto tem conseqüências no processo de filtração, ou seja, os componentes que são rejeitados acumulam-se perto da superfície da membrana. Este acúmulo próximo à superfície é definido como concentração polarizada, $\mathrm{c}_{\mathrm{w}}$, e tem conseqüências sérias, especialmente em ultrafiltração (BLATT, 1970 apud NOORDMAN, 2000). A Figura 4 ilustra a região de concentração polarizada.

O escoamento de fluido na corrente livre influencia a transferência de partículas de volta do acúmulo de soluto, assim a camada limite de concentração fica mais fina. (CHERYAN, 1998). A Figura 4 ilustra a região da camada limite de concentração, de espessura delgada, $\delta$, delimitada pela concentração polarizada e pela corrente livre. 
Se o regime de escoamento permite um grau de mistura muito alto (regime turbulento), as partículas rejeitadas são transportadas rapidamente de volta à corrente livre do escoamento e o acúmulo de componentes rejeitados, próximo à superfície da membrana, é limitado. Porém, se o regime de escoamento permite um grau de mistura baixo (regime laminar), o transporte de partículas de soluto rejeitadas não volta para a corrente livre rapidamente e o soluto rejeitado pode se acumular próximo à superfície a níveis indesejáveis. Este acúmulo continua até a concentração próxima à superfície ser muito alta e assim proporcionar para as partículas um alto potencial de difusão de volta à corrente livre (NOORDMAN, 2000).

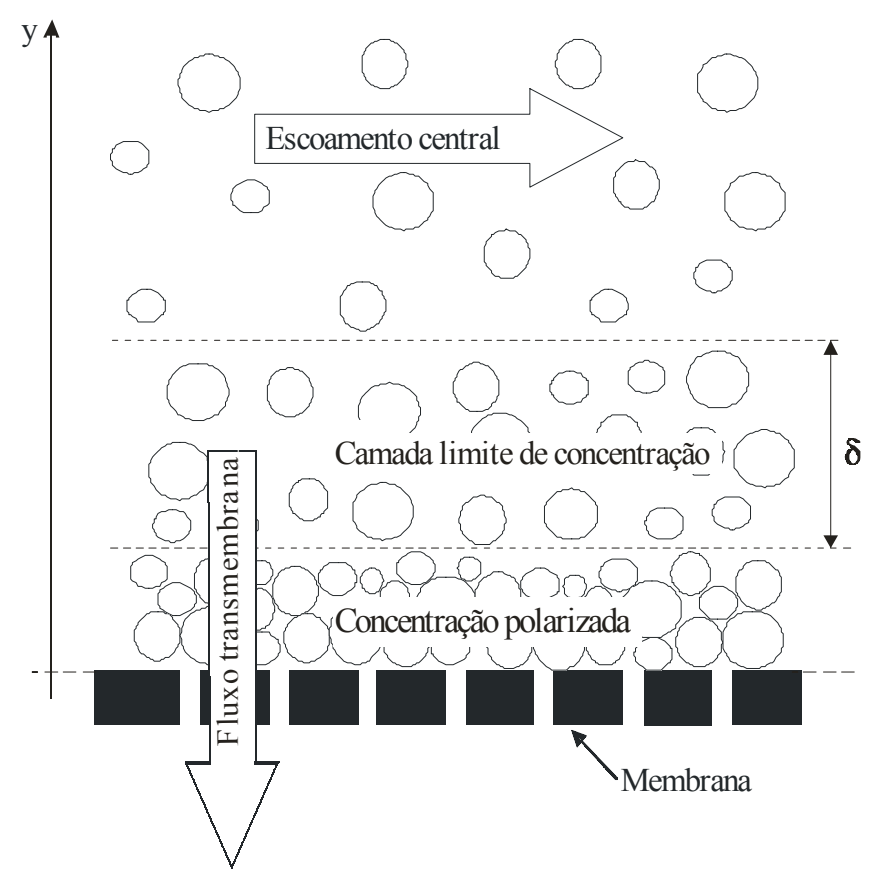

Figura 4. Diagrama esquemático da concentração polarizada e da camada limite de concentração, de espessura $\delta$, em filtração tangencial. Um gradiente de concentração é produzido através da camada limite de concentração. Temos também a indicação do fluxo transmembrana e do escoamento central.

Como visto na Figura 4, os processos de filtração podem ser divididos em duas regiões distintas de interesse no transporte de massa. Conforme veremos detalhadamente na seção seguinte, a primeira região refere-se à transferência de massa da fase liquida $(y>0)$; em particular, a convecção do soluto para o plano $\mathrm{y}=0$ e a correspondente difusão (de volta) do 
soluto da camada limite de concentração para o plano $\mathrm{y}=\delta$ (TRETTIN, 1980). A segunda região de interesse envolve o mecanismo de passagem do soluto e solvente através da própria membrana $(\mathrm{y}<0)$. Obviamente, uma completa análise na filtração por membrana envolverá uma modelagem combinada de ambas as regiões, unidas através da continuidade das espécies e da teoria dos mecanismos de transporte.

Na busca por explicações da dependência entre o fluxo e a pressão, em ultrafiltração de solutos macromoleculares, Bixler et al. (1968), Michaels (1968), Blatt et al. (1970) e Porter (1972) utilizaram os princípios da teoria do filme. Nesta teoria assume-se que o coeficiente de difusão (difusividade) é independente da concentração e os efeitos das variações da viscosidade são negligenciáveis. Supõe uma fina camada de fluido interfacial, onde não existe convecção axial, pelo qual ocorre todo transporte. A teoria do filme postula, por um lado, a presença de um filme estagnado de solução limitada pela membrana e, no lado oposto, uma região de concentração uniforme (corrente livre), na qual a transferência convectiva existe (TRETTIN, 1980). A teoria do filme é uma boa aproximação para os modelos relacionados aos processos de filtração tangencial, quando a camada limite de concentração é fina e uniforme (como nos escoamentos turbulentos) (CHERYAN, 1998).

\subsubsection{ConservaÇão da Massa na Superfície Permeável}

Considere um volume de controle, VC, bidimensional de tamanho constante e fixo no espaço. Um soluto (fluido) passa através das bordas do volume de controle e dentro deste volume iterações físico-químicas (ou pseudo-reações químicas) podem ocorrer (Figura 5). Assim uma situação dinâmica pode conduzir a mudanças na concentração dos vários componentes da mistura. Se examinarmos um soluto individual, podemos estabelecer a relação de conservação como: (TRUSKEY, 2004): 


$$
\left[\begin{array}{c}
\text { Taxa de acúmulo } \\
\text { de massa no VC }
\end{array}\right]=\left[\begin{array}{c}
\text { fluxo de massa } \\
\text { que entra no VC }
\end{array}\right]-\left[\begin{array}{c}
\text { fluxo de massa } \\
\text { que sai no VC }
\end{array}\right]+\left[\begin{array}{c}
\text { ganho ou perda } \\
\text { de massa na produção } \\
\text { de reações químicas }
\end{array}\right]
$$

No modelo do filme estagnado a taxa de acúmulo de massa no volume de controle é negligenciada e também se considera que não existe reação química na mesma. Assim a Equação (7) será escrita na forma:

$$
0=\left[\begin{array}{c}
\text { fluxo de massa } \\
\text { que entra no VC }
\end{array}\right]-\left[\begin{array}{c}
\text { fluxo de massa } \\
\text { que sai do VC }
\end{array}\right]
$$

Se fixarmos o volume de controle na interface da membrana, como pode ser visto na Figura 5, teremos o seguinte:

$$
\left[\begin{array}{c}
\text { fluxo de massa } \\
\text { que entra no VC }
\end{array}\right]=\left[\begin{array}{c}
\text { fluxo de massa que vai } \\
\text { em direção à membrana }
\end{array}\right]
$$

e também:

$$
\left[\begin{array}{c}
\text { fluxo de massa } \\
\text { que sai no VC }
\end{array}\right]=\left[\begin{array}{c}
\text { fluxo que volta } \\
\text { da membrana }
\end{array}\right]+\left[\begin{array}{c}
\text { fluxo que passa } \\
\text { pela membrana }
\end{array}\right]
$$

Substituindo as Equações (9) e (10) na Equação (8), obtemos (TRUSKEY, 2004):

$$
\underbrace{\left[\begin{array}{c}
\text { fluxo de massa que vai } \\
\text { em direção à membrana }
\end{array}\right]}_{\phi_{\mathrm{c}}}=\underbrace{\left[\begin{array}{c}
\text { fluxo que volta } \\
\text { da membrana }
\end{array}\right]}_{\phi_{\mathrm{d}}}+\underbrace{\left[\begin{array}{c}
\text { fluxo que passa } \\
\text { pela membrana }
\end{array}\right]}_{\mathrm{N}_{\mathrm{s}}}
$$

Assim temos que, respectivamente, $\phi_{\mathrm{c}}, \phi_{\mathrm{d}}$ e $\mathrm{N}_{\mathrm{s}}$ são o fluxo convectivo, o fluxo difusivo e o fluxo de soluto, os quais, para escoamentos numa superfície plana e permeável, são dados por:

$$
\begin{aligned}
& \phi_{\mathrm{c}}=-\mathrm{v}_{\mathrm{w}} \mathrm{c}_{\mathrm{w}} \\
& \phi_{\mathrm{d}}=-\left.\mathrm{D} \frac{\partial \mathrm{c}}{\partial \mathrm{y}}\right|_{\mathrm{y}=0}
\end{aligned}
$$




$$
\mathrm{N}_{\mathrm{S}}=-\mathrm{v}_{\mathrm{w}} \mathrm{c}_{\mathrm{f}}
$$

O sinal negativo na Equação (13) significa que a massa se desloca da maior para a menor concentração.

Na Figura 5 temos que $\hat{n}$ é o vetor normal à superfície e tem sentido positivo com relação ao eixo transversal, y, " $\vec{v}$ ” é o vetor velocidade transversal e oposto ao vetor normal. Vemos ainda que a velocidade na parede, $\mathrm{v}_{\mathrm{w}}$, é a intensidade de “ $\overrightarrow{\mathrm{v}}$ " e o fluxo transmembrana, J, também é paralelo e de mesmo sentido do vetor “ $\overrightarrow{\mathrm{v}}$ ".

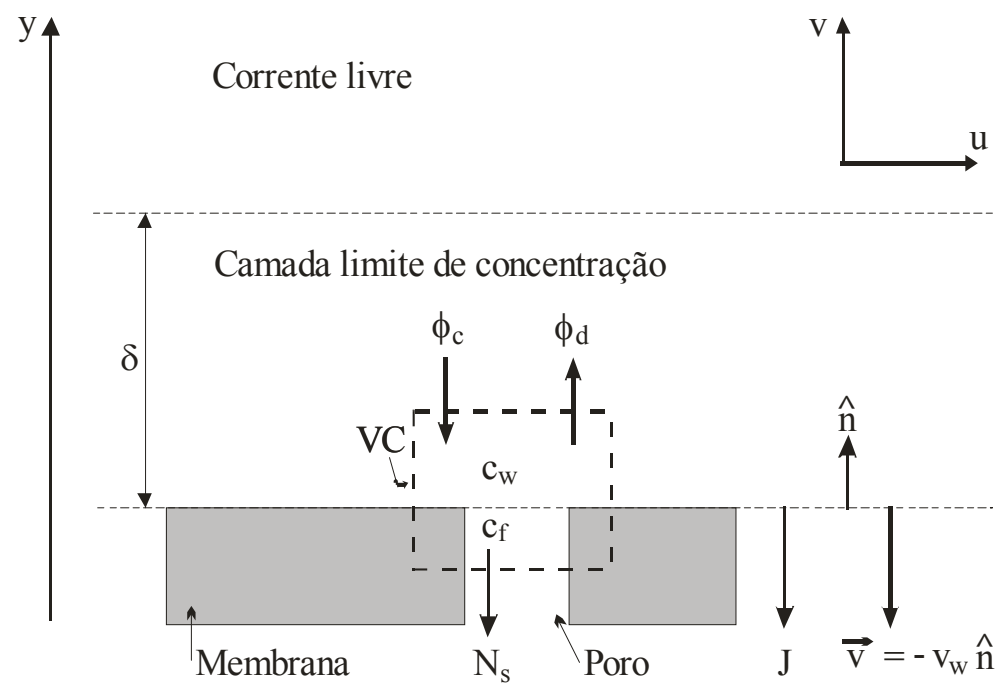

Figura 5. Desenho esquemático do balanço de massa na superfície permeável, onde " $\hat{n}$ " é o vetor unitário e normal à membrana, " $\overrightarrow{\mathrm{V}}$ " o vetor velocidade transversal (de intensidade " $\mathrm{v}_{\mathrm{w}}$ ", paralelo e oposto ao vetor normal). O fluxo transmembrana, J, também é paralelo ao vetor velocidade " $\vec{v}$ ". Indicamos "u" e "v", respectivamente, sendo a velocidade longitudinal e velocidade transversal.

Assim a Equação (11) pode ser escrita como:

$$
-\mathrm{v}_{\mathrm{w}} \mathrm{c}_{\mathrm{w}}=-\left.\mathrm{D} \frac{\partial \mathrm{c}}{\partial \mathrm{y}}\right|_{\mathrm{y}=0}-\mathrm{v}_{\mathrm{w}} \mathrm{c}_{\mathrm{f}}
$$

a qual é a clássica equação do filme estagnado.

Se considerarmos a completa rejeição das partículas pela superfície permeável, logo 
não teremos o fluxo de soluto através da membrana, isto é, $\mathrm{v}_{\mathrm{w}} \mathrm{c}_{\mathrm{f}}=0$. A Equação (15) pode ser escrita como:

$$
\mathrm{c}_{\mathrm{w}} \mathrm{v}_{\mathrm{w}}-\left.\mathrm{D} \frac{\partial \mathrm{c}}{\partial \mathrm{y}}\right|_{\mathrm{y}=0}=0
$$

Pela teoria do cálculo diferencial, temos a seguinte aproximação:

$$
\left.\frac{\partial \mathrm{c}}{\partial \mathrm{y}}\right|_{\mathrm{y}=0} \approx \frac{\mathrm{c}_{\mathrm{w}}-\mathrm{c}_{0}}{\delta(\mathrm{x})}
$$

Substituindo a Equação (17) na Equação (16), obtemos:

$$
\mathrm{c}_{\mathrm{w}}(\mathrm{x})=\frac{\mathrm{c}_{0}}{1-\frac{\delta(\mathrm{x})}{\mathrm{D}} \mathrm{v}_{\mathrm{w}}}
$$

com $0 \leq \mathrm{x} \leq \mathrm{L}$.

Uma expressão matemática para a espessura da camada limite de concentração será exposta na Seção (3.3).

\subsubsection{EQUAÇÃo CONVECTIVA-DIFUSIVA}

A equação convectiva-difusiva elíptica, cujas coordenadas em geometria cilíndrica são "x" e "r", para um escoamento de um fluido newtoniano em um tubo com superfície permeável, de comprimento " $L$ " e raio " $R_{0}$ ", regime laminar e estacionário, com densidade do soluto e difusividade constantes (TRETTIN, 1980), tem a forma:

$$
u \frac{\partial c}{\partial x}+v \frac{\partial c}{\partial r}=D\left(\frac{1}{r} \frac{\partial}{\partial r}\left(\frac{\partial c}{\partial r}\right)+\frac{\partial c^{2}}{\partial x^{2}}\right)
$$

com $0<\mathrm{x} \leq \mathrm{L}, 0<\mathrm{r} \leq \mathrm{R}_{0}, \mathrm{u} \equiv \mathrm{u}(\mathrm{x}, \mathrm{r}), \mathrm{v} \equiv \mathrm{v}(\mathrm{x}, \mathrm{r})$ e $\mathrm{c} \equiv \mathrm{c}(\mathrm{x}, \mathrm{r})$. Classificaremos a Equação 
(19) de acordo com as parcelas não negligenciadas, ou seja:

$$
\begin{aligned}
& \mathrm{u} \frac{\partial \mathrm{c}}{\partial \mathrm{x}}=\mathrm{D}\left(\frac{1}{\mathrm{r}} \frac{\partial}{\partial \mathrm{r}}\left(\frac{\partial \mathrm{c}}{\partial \mathrm{r}}\right)\right): \text { eq. axial-convectiva-difusiva parabólica; } \\
& \mathrm{u} \frac{\partial \mathrm{c}}{\partial \mathrm{x}}+\mathrm{v} \frac{\partial \mathrm{c}}{\partial \mathrm{r}}=\mathrm{D}\left(\frac{1}{\mathrm{r}} \frac{\partial}{\partial \mathrm{r}}\left(\frac{\partial \mathrm{c}}{\partial \mathrm{r}}\right)\right): \text { eq. convectiva-difusiva parabólica; }
\end{aligned}
$$

Essencialmente, os fluxos de suspensão são sistemas bifásicos em que o comportamento dinâmico do fluido é influenciado pelas interações microscópicas entre partículas sólidas e o escoamento do líquido. No entanto, não é possível incluir, por exemplo, a força de arrasto e os efeitos de interações nos modelos contínuos, devido à falta de técnicas práticas para medir tais fenômenos (RICHARDSON e NASSEHI, 2003). Desta forma, consideraremos no presente trabalho que o fluido e as partículas se movem com a mesma velocidade.

Muitos autores utilizaram a equação axial convectiva-difusiva parabólica (Equação (20)) para modelar e simular fenômenos de interesse. Elliot, Amidon e Lightfoot (1980) e Mansour (1988) simularam modelos de absorção acoplada com permeação através da parede intestinal com seção circular, pois, devido à velocidade de permeação, $\mathrm{v}_{\mathrm{w}}$, ser muito menor que a velocidade axial, $\mathrm{u}$, a parcela envolvendo a convecção radial adimensional, v $\partial \mathrm{c} / \partial \mathrm{r}$, pode ser negligenciada. Kumar, Pandey e Upadhyay (2000) obtêm resultados da concentração intracelular de íon de cálcio nas células endotelial de aorta de bezerros. Trettin (1980) determina o coeficiente de transferência de massa através desta equação. Dindore, Brilman e Versteeng (2005) modelam membranas modulares e analisam o comportamento do processo de transferência de massa.

A Equação (21) pode ser utilizada em processos de filtração tangencial, assim, se 
considerarmos o transporte de massa na direção axial, a contribuição do transporte difusivo, $\partial^{2} \mathrm{c} / \partial^{2} \mathrm{x}$, é muito menor que a contribuição do transporte convectivo, desta forma, a contribuição do transporte difusivo pode ser negligenciada. Wang, Deng e Guidoin (2003) utilizaram este fato para modelar e simular a concentração de polarização de macromoléculas em artérias carótida caninas. Paris et al. (2002) analisam o comportamento do transporte de massa, em tubos, do soluto dextrana T500. Trettin (1980), através da equação convectivadifusiva parabólica, modela o perfil de concentração e determina o coeficiente de transporte de massa nas adjacências da superfície permeável.

Damak et al. (2004; 2004A), através da Equação (19), investigam o efeito de vários parâmetros físicos sobre o perfil de concentração em toda extensão da membrana.

Zydney (1997) demonstra que a difusividade é dependente da concentração e deve ser incorporada no formalismo do filme estagnado e esta dependência foi utilizada por muitos autores, tais como, Paris et al. (2002), Yeh et al. (2004), Geraldes et al. (2001) e Romero et al. (1988). Contudo, nos trabalhos de Boyadjiev (2006), Wang et al. (2003) e Damak et al. (2004), a difusividade foi considerada constante.

As condições de contorno, de entrada e de saída para as Equações de (19) até (21) são:

- na entrada:

$$
\mathrm{c}(0, \mathrm{r})=\mathrm{c}_{0}, \quad 0 \leq \mathrm{r} \leq \mathrm{R}_{0}
$$

- no eixo aximétrico:

$$
\mathrm{c}(\mathrm{x}, 0)=\mathrm{c}_{0}, \quad 0 \leq \mathrm{x} \leq \mathrm{L}
$$


- na parede permeável:

$$
\mathrm{c}\left(\mathrm{x}, \mathrm{R}_{0}\right)=\mathrm{c}_{\mathrm{w}}(\mathrm{x}), \quad 0 \leq \mathrm{x} \leq \mathrm{L}
$$

- na saída:

$$
\left.\frac{\partial \mathrm{c}(\mathrm{x}, \mathrm{r})}{\partial \mathrm{x}}\right|_{\mathrm{x}=\mathrm{L}}=0, \quad 0 \leq \mathrm{r} \leq \mathrm{R}_{0}
$$

A Figura 6 mostra as condições de contorno na interface da superfície permeável do tubo, $r=R_{0}$, no eixo de simetria do tubo, $r=0$, a condição de entrada, $x=0$. A concentração de entrada, $\mathrm{c}_{0}$, e a velocidade média de entrada, $\mathrm{u}_{0}$, são consideradas uniformes em $0 \leq \mathrm{r} \leq \mathrm{R}_{0}$. O perfil de concentração, com relação ao eixo radial, varia dentro da camada limite de concentração de espessura, $\delta$, mas é uniforme fora desta região. A velocidade na parede, $\mathrm{v}_{\mathrm{w}}$, é também considerada uniforme, contudo o fluxo transmembrana local, $\mathrm{J} \equiv \mathrm{J}(\mathrm{x})$, varia com a posição axial.

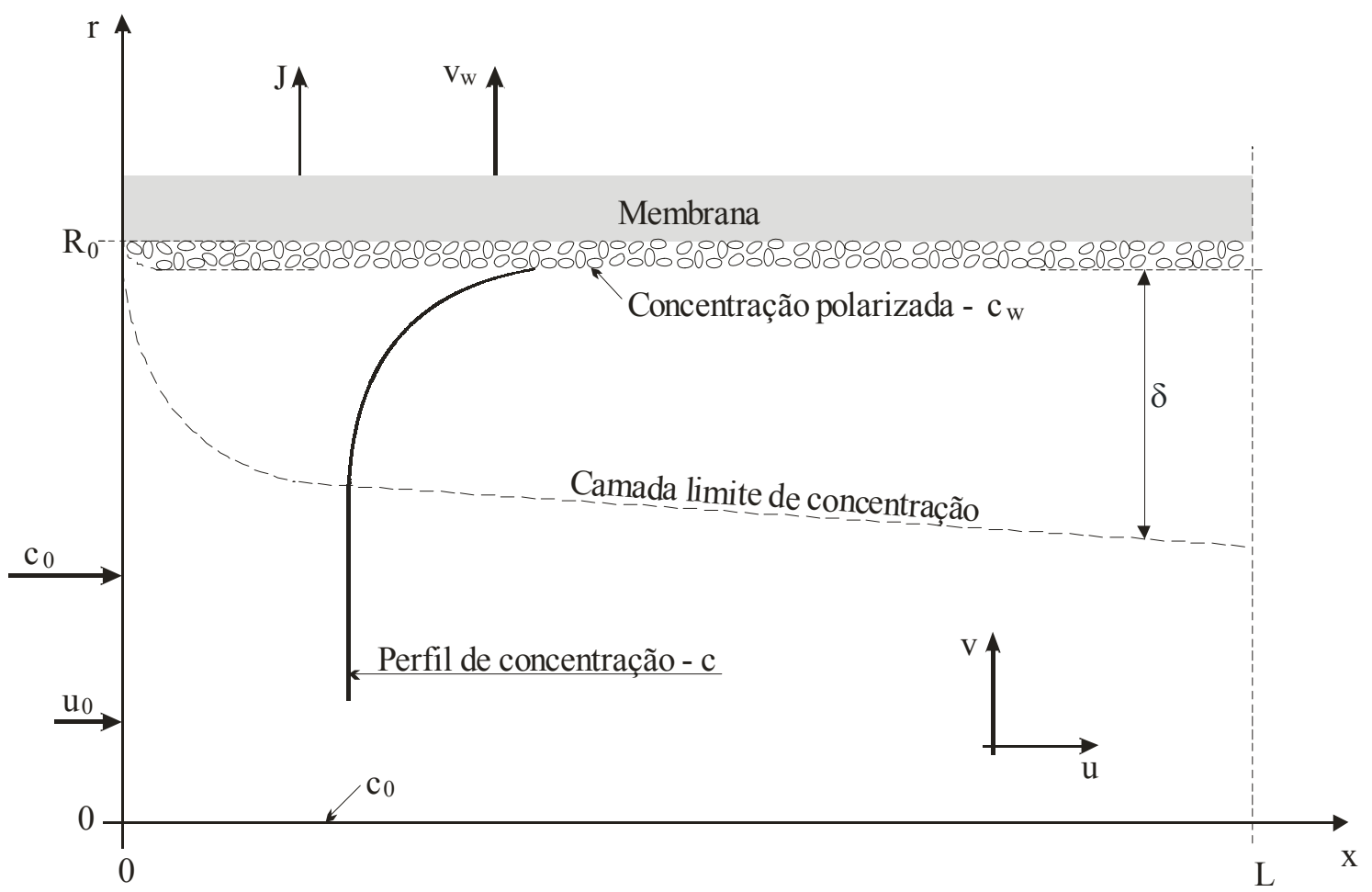

Figura 6. Esquema da condição de contorno e entrada de um tubo permeável de comprimento "L" e raio " $\mathrm{R}_{0}$ ", para um escoamento em regime estacionário e laminar de um fluido incompressível e newtoniano. 
Na teoria clássica do filme, vista na Seção (2.1.4), considera-se, como condição de contorno, que a velocidade de permeação é equivalente ao fluxo transmembrana em toda extensão do filme estagnado, ou seja:

$$
\mathrm{v}_{\mathrm{w}}=\mathrm{J}, \quad 0 \leq \mathrm{x} \leq \mathrm{L}
$$

Utilizaremos a Equação (26) na determinação de uma específica taxa, e tal metodologia será vista na Seção (3.3.2).

$\mathrm{Na}$ condição de contorno na parede $\left(\mathrm{r}=\mathrm{R}_{0}\right)$, estabelecida pela Equação (24), temos a concentração na superfície permeável variando com a posição axial, a qual está definida na Equação (18).

\subsubsection{EQUAÇÕES DE MOMENTUM}

O efeito da filtração transmembrana, em um tubo com superfície permeável, de um escoamento em regime laminar foi investigado, por vários autores, através da solução das equações de Navier-Stokes.

Vários modelos foram propostos para determinado escoamento laminar de um fluido em canais e tubos porosos com injeção ou sucção na parede (OXARANGO et al., 2004). Duas categorias de problemas são apresentadas na literatura: (i) escoamento completamente desenvolvido, onde as formas dos perfis de velocidade são consideradas similares, e (ii) escoamento em desenvolvimento, onde a forma do perfil de velocidade varia com a posição axial. Além disso, muitos autores têm assumido valores conhecidos da velocidade na parede permeável (velocidade de permeação) ser dada a priori (OXARANGO et al., 2004). Contudo, em muitos sistemas reais, esta última suposição não é valida, visto que a velocidade de filtração na parede, $\mathrm{v}_{\mathrm{w}}$, é movida pela pressão local na parede que varia com a posição axial. Considerando uniforme a velocidade de permeação na parede, fica subentendido que 
variações de pressão deveriam existir fora do canal ou que a permeabilidade da parede porosa varia ao longo do canal (permeabilidade variando com a posição axial).

Soluções de perfis de velocidade similares foram encontradas por muitos autores (BERMAN, 1953; YUAN, FINKELSTEIN e BROOKLYN, 1956; TERRIL, 1964). Considerando uma velocidade de sucção ou injeção uniforme, Brandy (1984) mostrou que o escoamento dentro do canal (ou do tubo) é determinado pela condição de entrada, onde o número de Reynolds na parede, $\mathrm{Re}_{\mathrm{w}}$, está acima de um valor crítico, 2,3 para o caso de tubos (axisimétricos) e 6 para o caso de canais. Conseqüentemente, concluiu-se que as soluções para perfis similares é aproximada para $\mathrm{Re}_{\mathrm{w}}$ suficientemente pequenos (BRANDY, 1984). Além disso, neste caso, as soluções derivadas de Berman (1953), para canais bidimensionais, e Yuan, Finkelstein e Brooklyn (1956) para tubos, são válidas para pequenos valores de "Re $\mathrm{w}_{\mathrm{w}}$ ". Contudo, as soluções de Berman (1953) foram desenvolvidas considerando-se a velocidade de filtração na parede, $\mathrm{v}_{\mathrm{w}}$, sendo uniforme (OXARANGO et al., 2004).

Muitos autores (WEISSBERG, 1959; QUAILE e LEVY, 1975 apud OXARANGO et al., 2004) determinaram soluções de escoamentos em desenvolvimento. Correspondentes estudos confirmam a existência de uma região onde não é válido considerar o perfil de velocidade simétrico. Contudo, tais regiões são limitadas pela entrada do canal (ou tubo) e elas podem geralmente ser omitidas para um número muito grande de canais, ou tubos (OXARANGO et al., 2004).

A equação da continuidade e as equações de momentum adimensionais em coordenadas cilíndricas $(\mathrm{X}, \mathrm{R})$ que modelam um escoamento em regime laminar em desenvolvimento e estacionário, em tubo com superfície porosa de comprimento $\mathrm{L}$ e raio $\mathrm{R}_{0}$, para um fluido incompressivel, são: 


$$
\begin{aligned}
& \frac{\partial(\mathrm{R} \mathrm{V})}{\partial \mathrm{R}}+\frac{\partial \mathrm{U}}{\partial \mathrm{X}}=0 \\
& \mathrm{~V} \frac{\partial \mathrm{U}}{\partial \mathrm{R}}+\mathrm{U} \frac{\partial \mathrm{U}}{\partial \mathrm{X}}=-\frac{\partial \mathrm{P}}{\partial \mathrm{X}}+\frac{1}{\mathrm{Re}}\left[\frac{\partial}{\partial \mathrm{R}}\left(\frac{1}{\mathrm{R}} \frac{\partial(\mathrm{R} U)}{\partial \mathrm{R}}\right)+\frac{\partial^{2} \mathrm{U}}{\partial \mathrm{X}^{2}}\right] \\
& \mathrm{V} \frac{\partial \mathrm{V}}{\partial \mathrm{R}}+\mathrm{U} \frac{\partial \mathrm{V}}{\partial \mathrm{X}}=-\frac{\partial \mathrm{P}}{\partial \mathrm{R}}+\frac{1}{\mathrm{Re}}\left[\frac{\partial}{\partial \mathrm{R}}\left(\frac{1}{\mathrm{R}} \frac{\partial(\mathrm{R} V)}{\partial \mathrm{R}}\right)+\frac{\partial^{2} \mathrm{~V}}{\partial \mathrm{X}^{2}}\right]
\end{aligned}
$$

sendo $\mathrm{U} \equiv \mathrm{U}(\mathrm{X}, \mathrm{R}), \mathrm{V} \equiv \mathrm{V}(\mathrm{X}, \mathrm{R}), \quad 0 \leq \mathrm{R} \leq 1, \quad 0 \leq \mathrm{X} \leq \xi, \quad \xi=\mathrm{L} / \mathrm{R}_{0}$ com as condições de fronteira:

- no centro do tubo, $\mathrm{R}=0,0 \leq \mathrm{X} \leq \xi$ :

$$
\left.\frac{\partial \mathrm{U}(\mathrm{X}, \mathrm{R})}{\partial \mathrm{R}}\right|_{\mathrm{R}=0}=0 \quad \text { e } \quad \mathrm{V}(\mathrm{X}, 0)=0
$$

- na parede do tubo, $\mathrm{R}=1,0 \leq \mathrm{X} \leq \xi$ :

$$
\mathrm{U}(\mathrm{X}, 1)=0 \quad \text { e } \quad \mathrm{V}(\mathrm{X}, 1)=\mathrm{V}_{\mathrm{w}}(\mathrm{X})
$$

- na entrada do tubo, $\mathrm{X}=0,0 \leq \mathrm{R} \leq 1$ :

$$
\mathrm{U}(0, \mathrm{R})=\left(1-\mathrm{R}^{2}\right) \quad \text { e } \quad \mathrm{V}(0, \mathrm{R})=0
$$

- na saída do tubo, $\mathrm{X}=\xi, 0 \leq \mathrm{R} \leq 1$ :

$$
\left.\frac{\partial \mathrm{U}(\mathrm{X}, \mathrm{R})}{\partial \mathrm{X}}\right|_{\mathrm{X}=\xi}=0 \quad \text { e }\left.\quad \frac{\partial \mathrm{V}(\mathrm{X}, \mathrm{R})}{\partial \mathrm{X}}\right|_{\mathrm{X}=\xi}=0
$$

Os trabalhos de Berman (1953) e Yuan, Finkelstein e Brooklyn (1956) apresentam uma solução aproximada do perfil de velocidade similar para um escoamento em um tubo com superfície porosa, com velocidade uniforme na parede e um número de Reynolds de filtração, $\mathrm{Re}_{\mathrm{w}}$, suficientemente pequeno (BRANDY, 1984), e esta é uma situação encontrada em muitos dos processos de filtração (OXARANGO et al., 2004). 


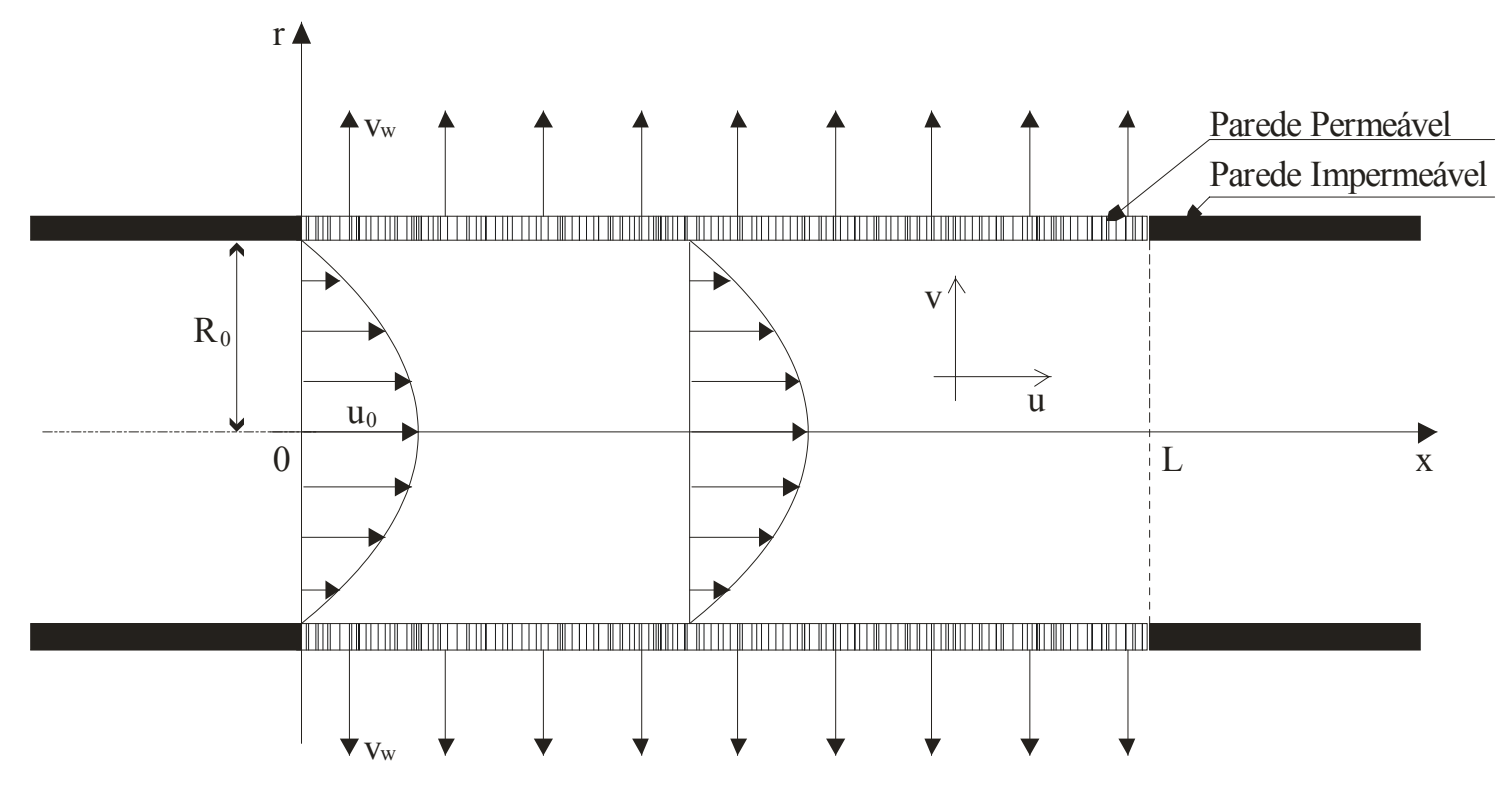

Figura 7. Representação do escoamento laminar completamente desenvolvido em tubo com superfície permeável.

No trabalho de Yuan, Funkelstein e Brooklyn (1956) tem-se uma solução aproximada das equações de Navier-Stokes em coordenadas cilíndricas de um escoamento laminar e estacionário em tubo com superfície permeável (Figura 7). Esta solução é obtida através da redução das equações de Navier-Stokes para uma equação diferencial não linear de terceira ordem com apropriadas condições de contorno. Um método de perturbação foi usado para resolver esta equação, para pequenos e grandes fluxos através da parede porosa. Os componentes de velocidade são expressos como funções da taxa de velocidade através da parede porosa para a velocidade axial máxima na entrada de tubo, da coordenada do tubo, e das propriedades físicas do fluido.

As expressões para os perfis de velocidades axial e radial adimensionais, são dadas por (YUAN, FINKELSTEIN e BROOKLYN, 1956):

$$
\begin{aligned}
U_{1}(R)=\left[1-R^{2}+\right. & \frac{R e_{w}}{36}\left(-2+9 R^{2}-9 R^{4}+2 R^{5}\right)+\frac{R_{w}^{2}}{10800}\left(166-760 R^{2}+825 R^{4}-300 R^{5}+\right. \\
& \left.\left.+75 R^{6}-6 R^{7}\right)\right]
\end{aligned}
$$




$$
\begin{aligned}
\mathrm{U}_{2}(\mathrm{X})=\left[\frac{1}{1-\frac{\mathrm{Re}_{\mathrm{w}}}{18}+\frac{83}{5400} \mathrm{Re}_{\mathrm{w}}^{2}}+4 \frac{\mathrm{Re}_{\mathrm{w}}}{\mathrm{Re}} \mathrm{X}\right] \\
\mathrm{V}(\mathrm{R})=-\frac{2 \mathrm{Re}_{\mathrm{w}}}{\operatorname{ReR}^{\mathrm{R}}}\left[\mathrm{R}^{2}-\frac{1}{2} \mathrm{R}^{4}+\frac{\mathrm{Re}_{\mathrm{w}}}{72}\left(-4 \mathrm{R}^{2}+9 \mathrm{R}^{4}-6 \mathrm{R}^{5}+\mathrm{R}^{6}\right)+\right. \\
\left.+\frac{\operatorname{Re}_{\mathrm{w}}^{2}}{10800}\left(166 \mathrm{R}^{2}-380 \mathrm{R}^{4}+275 \mathrm{R}^{5}-75 \mathrm{R}^{6}+15 \mathrm{R}^{7}-\mathrm{R}^{8}\right)\right]
\end{aligned}
$$

com $U(X, R)=U_{1}(R) U_{2}(X)$. Esta separação de variáveis da velocidade axial adimensional, U, foi utilizada visado o tratamento analítico da transformada integral, conforme veremos na Equação (69) até a Equação (78).

Os perfis de velocidade dados pelas Equações (34), (35) e (36) também foram usadas por Oxarango et al. (2004) e Wang et al. (2003).

Considerando a pressão de entrada, $\mathrm{p}_{0}$, uniforme, a pressão local na direção axial é dada por (YUAN, FINKELSTEIN e BROOKLYN, 1956):

$$
\mathrm{p}(\mathrm{X})=\mathrm{p}_{0}-\frac{4 \rho \mathrm{u}_{0}^{2}}{\operatorname{Re}}\left[1+\frac{3}{4} \operatorname{Re}_{\mathrm{w}}-\frac{11}{270} \operatorname{Re}_{\mathrm{w}}^{2}\right]\left[\frac{1}{1-\frac{\operatorname{Re}_{\mathrm{w}}}{18}+\frac{83}{5400} \operatorname{Re}_{\mathrm{w}}^{2}}+2 \frac{\operatorname{Re}_{\mathrm{w}}}{\operatorname{Re}} \mathrm{X}\right] \mathrm{X}
$$

\subsubsection{Entupimento dA MEMbrana (Fouling)}

O entupimento da membrana (fouling) é um fenômeno pelo qual a membrana interage física e quimicamente com o soluto presente na corrente principal, o qual causa um decrescimento no desempenho da membrana, isto é, diminuição da vazão e aumento na rejeição do soluto (CHERYAN, 1998).

Nos efeitos de entupimento (fouling), a vazão de filtrado e soluto passam a ter variações 
semelhantes àquelas associadas com o fenômeno da camada de polarização. No entanto, a concentração de polarização é um fenômeno na camada limite reversível. Assim, a concentração de polarização pode ser controlada com condições de operação de fluxo reverso. Esses efeitos podem ser reduzidos ou até eliminados pelo aumento da tensão de cisalhamento do fluido na superfície permeável (ZEMAN e ZYDNEY, 1996).

O entupimento ocorre porque interações (atrativas) físicas e/ou químicas ocorrem entre os vários macrosolutos, ou partículas, e a membrana. A taxa e alcance do entupimento é tipicamente uma função do dispositivo fluido mecânico, mas não pode, em geral, ser eliminada simplesmente pelo acréscimo da taxa de transferência de massa do soluto.

O entupimento (fouling) permanece um fenômeno pouco entendido. Em princípio, todos os componentes presentes no processo de filtração podem obstruir a membrana. A natureza e o alcance do entupimento são determinados pelas características físico-químicas moleculares dos componentes individuais e da membrana. Em muitos sistemas, as espécies que causam os efeitos dominantes do entupimento estão presentes em pequenas quantidades, e suas concentrações podem ser independentes dos principais componentes envolvidos no processo de filtração.

Um dos maiores problemas relacionados ao entendimento do mecanismo de formação do entupimento (fouling) é distingui-lo dos efeitos da concentração de polarização. Muitos pesquisadores têm atribuído o rápido declínio da vazão como o começo do processo de polarização. Qualquer redução sistemática da vazão transmembrana é então atribuída ao entupimento da membrana. No entanto, este entupimento também pode desenvolver-se muito rapidamente, por exemplo, o tempo característico para a adsorção de proteínas em superfícies sólidas pode geralmente ser de apenas alguns segundos. Mudanças físicas na estrutura da membrana podem ser causadas por compactação ou deformação. A degradação química 
também pode ser um problema em muitos sistemas, se o $\mathrm{pH}$, temperatura, ou composição química da solução ou agentes de limpeza forem incompatíveis com a composição química da membrana (ZEMAN e ZYDNEY, 1996).

\subsubsection{Modelo da Camada de Gel Polarizado e Modelo da ResistênCia em Série}

Existem na literatura várias relações quantitativas que correlacionam o coeficiente de transferência de massa com propriedades físicas, escoamento e parâmetros operacionais. Nenhuma é completamente satisfatória e muitas delas estão sendo continuamente refinadas ou modificadas para se encaixarem as particulares aplicações, e desta forma, não são aplicáveis universalmente (CHERYAN, 1998). Freqüentemente são utilizados os modelos do gel polarizado e da resistência em série.

O modelo do filme estagnado (visto no final da Seção (2.1.4)) foi desenvolvido para simplificar a análise do transporte de massa de soluto em membranas reais. Obtém-se uma resolução aproximada para a equação de conservação das espécies químicas baseando-se no conceito de coeficiente de transferência de massa por convecção na superfície da membrana (ZEMAN e ZYDNEY, 1996), que é definido, localmente, como segue:

$$
\mathrm{k}(\mathrm{x})=\left.\frac{\mathrm{D}}{\mathrm{c}_{\mathrm{w}}(\mathrm{x})-\mathrm{c}_{0}} \frac{\partial \mathrm{c}}{\partial \mathrm{r}}(\mathrm{r}, \mathrm{x})\right|_{\mathrm{r}=\mathrm{R}_{0}}
$$

A literatura apresenta várias investigações (PARIS et al., 2002; NAKAO et al., 1979; ZYDNEY, 1997) na tentativa de quantificar a camada de concentração polarizada e avaliar a taxa de permeação em sistemas de filtração tangencial. Um estudo representativo foi o desenvolvimento do modelo da camada gel polarizado, o qual foi usado como ferramenta para interpretar dados experimentais em ultrafiltração e como base para o desenvolvimento teórico adicional em microfiltração. Posteriormente, as forças de cisalhamento e difusas foram inseridas no modelo para obter melhores ajustes com os dados experimentais. A teoria da 
camada de gel deriva da equação de balanço de massa (SONG e ELIMELECH, 1995), com duas grandezas desconhecidas: a velocidade de permeação e a distribuição de concentração de partículas sobre a membrana, ou seja:

$$
\mathrm{J}_{\lim }=\mathrm{k} \ln \left(\frac{\mathrm{c}_{\mathrm{w} 0}}{\mathrm{c}_{0}}\right)
$$

com "ln" sendo o logaritmo natural.

A partir da Equação (39) é impossível avaliar a velocidade de permeação sem a utilização de hipóteses adicionais. Esta teoria se torna um modelo semi-empírico que assume fixa a concentração de partículas na superfície permeável, $\mathrm{c}_{\mathrm{w} 0}$, e adapta um coeficiente de transferência de massa da teoria de transferência, convectiva de calor para superfícies impermeáveis. As aplicações práticas do modelo da camada de gel são limitadas; o modelo não pode ser usado para condições onde a velocidade de permeação é dependente da pressão (SONG e ELIMELECH, 1995), porém foi desenvolvido para prever o denominado fluxo crítico (BACHIN e AIMAR, 2006).

O modelo da camada gel polarizada (Equação (39)) estabelece uma descrição alternativa do fluxo de filtrado durante a ultrafiltração de macro-soluto (proteína). O mecanismo pelo qual o soluto acumulado reduz o fluxo de filtrado tem importância secundária no modelo da camada gel polarizada. Neste modelo postula-se que, em alta pressão transmembrana, $\Delta \mathrm{p}$, a concentração de soluto na superfície da membrana atinge um valor constante (máximo) que é determinado pelas características físicas de uma macromolécula particular. É geralmente suposto que esta máxima concentração reflete no ponto de gelificação, precipitação, e/ou agregação das macromoléculas na superfície da membrana (ZEMAN e ZYDNEY, 1996).

Durante a filtração tangencial, o modelo da camada de gel polarizado não descreve 
completamente o comportamento do fluxo relacionado à pressão. O modelo de resistência em série pode ser usado como uma outra aproximação para o fluxo transmembrana. A lei de Darcy (Equação (1)) determina o fluxo transmembrana de um solvente para uma membrana ideal, e pode ser escrita como:

$$
\mathrm{J}=\frac{\Delta \mathrm{p}}{\mathrm{R}_{\mathrm{m}}}
$$

No uso de soluções (soluto e solvente) nos processos de filtração tangencial, a resistência da membrana é apenas uma parte da resistência total aplicada sobre o fluxo transmembrana. A resistência do entupimento da membrana (fouling), $\mathrm{R}_{\mathrm{e}}$, é uma adicional resistência ao fluxo de permeado, a qual é incorporada ao modelo (Equação (40)) (CHERYAN, 1998):

$$
\mathrm{J}=\frac{\Delta \mathrm{p}}{\mathrm{R}_{\mathrm{m}}+\mathrm{R}_{\mathrm{e}}}
$$

A parcela de resistência da concentração polarizada e da camada limite de concentração, $\mathrm{R}_{\mathrm{g}}$, também é adicionada ao modelo do fluxo:

$$
J=\frac{\Delta p}{R_{m}+R_{e}+R_{g}}
$$

“ $\mathrm{R}_{\mathrm{g}}$ ” é uma função de parâmetros operacionais e propriedades físicas, assim ele será uma função que depende da permeabilidade do gel e sua espessura (e estas últimas são dadas em função da pressão transmembrana aplicada) (CHERYAN, 1998). Assim: $\mathrm{R}_{\mathrm{g}}=\phi \Delta \mathrm{p} . \mathrm{O}$ termo $\phi$ depende das variáveis que afetam a transferência de massa, tais como a viscosidade, a taxa de cisalhamento e a temperatura. O fluxo de filtrado, inicialmente, aumenta com o aumento da pressão transmembrana, mas em altas pressões a resistência da concentração polarizada é considerada proporcional a $\Delta \mathrm{p}$. Desta forma, o fluxo de filtrado atinge um valor máximo independente da pressão. No trabalho de Yeh et al. (2004) é usado o modelo da 
resistência em série e o mesmo é comparado com resultados experimentais.

Se a camada limite de concentração polarizada é, por hipótese, homogênea, a resistência da concentração polarizada é dada por: $\mathrm{R}_{\mathrm{g}}=\mathrm{r}_{\mathrm{g}} \delta_{\mathrm{g}}$, com $\mathrm{r}_{\mathrm{g}}$ sendo a resistência específica obtida através da equação de Carman-Kozeny (DAMAK et al., 2004; PARIS et al., 2002).

O fluxo transmembrana dado pela Equação (42) pode ser determinado localmente pela expressão: $\mathrm{J}(\mathrm{x})=\Delta \mathrm{p}(\mathrm{x}) /\left(\mathrm{R}_{\mathrm{m}}+\mathrm{R}_{\mathrm{e}}+\mathrm{R}_{\mathrm{g}}(\mathrm{x})\right), 0 \leq \mathrm{x} \leq \mathrm{L}$.

\subsubsection{CORRELAÇÃo DE SHERWOOD}

Podemos analisar, a partir do perfil de concentração, o comportamento do gradiente de concentração na parede, ou seja, estabelecemos uma medida da transferência de massa por convecção na superfície permeável e esta medida é caracterizada pelo valor do número de Sherwood local, dado pela expressão (DAMAK et al., 2004):

$$
\operatorname{Sh}(\mathrm{x})=\left.\frac{1}{\mathrm{C}_{\mathrm{w}}-1} \frac{\partial \mathrm{C}}{\partial \mathrm{R}}\right|_{\mathrm{R}=1}
$$

O coeficiente de transferência de massa é convenientemente expresso na clássica forma adimensional como (ZEMAN e ZYDNEY, 1996):

$$
\mathrm{Sh}=\frac{\mathrm{kR}_{0}}{\mathrm{D}}=\mathrm{w} \operatorname{Re}^{\alpha} \mathrm{Sc}^{\zeta}\left(\frac{\mathrm{R}_{0}}{\mathrm{~L}}\right)^{\gamma}
$$

com $\mathrm{w}, \alpha, \zeta$ e $\gamma$ sendo parâmetros que devem ser determinados empiricamente.

A correlação de Leveque (ZEMAN e ZYDNEY, 1996):

$$
\mathrm{Sh}=1,62\left(\frac{\mathrm{R}_{0}}{\mathrm{x}}\right)^{0,33} \mathrm{Re}^{0,33} \mathrm{Sc}^{0,33}
$$


não foi desenvolvida para filtração tangencial, mas para transferência de massa em sistemas impermeáveis. Contudo, ela é uma equação interessante, com a qual podemos comparar outros resultados provindos da filtração tangencial.

Em sistemas onde as propriedades do fluido e do escoamento são conhecidas, podem ser desenvolvidas relações empíricas baseadas na correlação de Gilland-Sherwood, que é aplicada para descrever o transporte de massa através da camada limite de concentração para escoamentos laminares (WELTY, WICKS e WILSON, 1969):

$$
\mathrm{Sh}=\mathrm{s}+\mathrm{w} \mathrm{Re}^{\alpha} \mathrm{Sc}^{\zeta}
$$

com s, w, $\alpha$ e $\zeta$ sendo parâmetros que devem ser determinados empiricamente.

A Equação (43) provém da expressão do coeficiente de transporte de massa, estabelecido pela teoria do filme estagnado, onde a concentração na parede permeável é considerada homogênea e uniforme. Contudo, podemos impor nesta equação que a concentração polarizada varie com a posição axial.

A partir das Equações (39) e (43), o fluxo transmembrana local é dado pelo modelo do gel polarizado:

$$
\mathrm{J}(\mathrm{x})=\mathrm{k}(\mathrm{x}) \ln \left(\frac{\mathrm{c}_{\mathrm{w} 0}}{\mathrm{c}_{0}}\right)
$$

sendo $\mathrm{k}(\mathrm{x})=\mathrm{D} \mathrm{Sh}(\mathrm{x}) / \mathrm{R}_{0}$ o coeficiente de transferência de massa local escrito na sua forma dimensional. Como o modelo do gel (Equação (47)) provém da teoria do filme estagnado, onde a concentração na parede permeável é considerada homogênea e uniforme, logo podemos considerar a concentração polarizada dada por: $c_{w 0}=\frac{1}{L} \int_{0}^{L} c(x) d x$. 


\subsection{Modelagem dos Processos de Filtração Tangencial}

Apesar do emprego cada vez mais freqüente, os processos de separação por membrana ainda não têm equações gerais envolvendo todos os mecanismos que regem os escoamentos em membranas, devido à complexidade dos fatores envolvidos. Desta forma, alguns autores apresentam modelos matemáticos empíricos que permitem predizer o declínio do fluxo de permeado com relação ao tempo; por exemplo, os modelos revisados por Cheryan (1986), Merin e Cheryan (1980 apud FARRO, 2003) e Wu et al. (1991 apud FARRO, 2003). Por outro lado, o desenvolvimento de modelos puramente matemáticos, bem como suas soluções, são muito utilizados na predição de fenômenos da filtração tangencial, tais como velocidade do fluxo permeado e resistência da camada gel polarizada, com bons resultados numéricos, quando comparados com os experimentos.

$\mathrm{Na}$ teoria clássica do filme, utilizando-se a equação convectiva-difusiva, consegue-se boa aproximação dos valores numéricos aos valores experimentais para problemas de transporte de massa, e isto pode ser visto no trabalho de Trettin (1980).

A modelagem de um mecanismo de transferência de massa por convecção é estabelecida por três equações: (i) equação da continuidade, (ii) equação do movimento e (iii) equação do transporte de massa, conservação das espécies químicas, ou equação convectivadifusiva. A busca de soluções simultâneas das referidas equações pode gerar resultados numéricos próximos dos valores experimentais.

Veremos, a seguir, diversos trabalhos envolvendo modelos matemáticos, tratados pela literatura, e relacionados aos processos de filtração tangencial, onde a partir dos mesmos procurou-se expor: (a) o problema a ser modelado, (b) modelagem e as condições de contorno e de entrada, (c) método utilizado na solução do modelo e (d) conclusão. 
Os modelos clássicos geralmente usados para estudar o problema do escoamento de fluidos em sistemas de filtração de multicanais somente se aplicam para um elemento de unidade representativo do sistema, isto é, um canal poroso simples com sucção na parede, no caso de canal de entrada, ou injeção na parede, no caso de canal de saída. A hipótese fundamental nestes modelos, que justificam o desacoplamento destas três regiões (a entrada, a saída e a parede porosa), é que o escoamento ao longo do tubo axial e dentro da região porosa pode ser negligenciado (OXARANGO et al., 2004).

Oxarango et al. (2004) estudam dois problemas diferentes correspondentes a canais e a tubos com superfícies permeáveis, contudo exporemos apenas o caso cilíndrico. Assim, é considerado um escoamento em regime laminar, completamente desenvolvido, de um fluido viscoso, newtoniano, incompressível em um tubo poroso bidimensional com sucção ou injeção uniforme. As equações que governam o problema são as equações de Navier-Stokes (OXARANGO, 2004) e estão sujeitas às seguintes condições: (a) na parede, a velocidade de permeação é determinada utilizando a equação de Darcy e a velocidade axial é nula e (b) no eixo aximétrico a variação da velocidade axial é nula e a velocidade radial também é nula.

A solução do modelo para um escoamento em tubos porosos com sucção uniforme ou injeção constante, para número de Reynolds suficientemente pequeno, é similar ao desenvolvido por Yuan e Finkelstein (1956, apud OXALANGO et al., 2004), que gera um conjunto de equações diferenciais ordinárias de segunda ordem e não linear, cujas funções destas são dadas pela velocidade média e a pressão média. Este conjunto de equações pode ser resolvido pelo método das diferenças finitas e o método de Newton-Raphson para os termos não lineares (OXARANGO et al., 2004). Este modelo preserva as mais importantes propriedades do escoamento; em particular, os termos inerciais, que podem afetar a condição de sucção na parede e a distribuição espacial das partículas escoando da camada de gel, como 
encontrado nos processos de filtração. O modelo é aplicado para sistemas de filtração de multicanais bidimensionais, podendo ser estendidos para tridimensionais, que consiste de um grande número de entradas e saídas adjacentes de canais conectados por um filtro de meio poroso.

Em processos de filtração tangencial, o fenômeno de polarização da concentração em estado estacionário, pode ser descrito pela equação convectiva-difusiva elíptica bidimensional, acoplada com o modelo de resistência em série para o transporte de permeação (DAMAK et al., 2004). O fluxo do alimentador, que escoa tangencialmente à superfície da membrana porosa, é modelado pelas equações de Navier-Stokes. O problema analisado por Damak et al. (2004) é o fenômeno de transferência de massa em tubo poroso em regime laminar, cujas coordenadas são (z,r). A análise é baseada nas seguintes hipóteses: (a) a viscosidade e a densidade são constantes, (b) o coeficiente de difusão é considerado constante, (c) o fluido é incompressível e as condições de regime permanentes são controladas, (d) o movimento é considerado como axisimétrico; assim, somente metade do tubo é considerada, (e) na entrada do tubo é considerado um perfil laminar completamente desenvolvido; assim, a velocidade axial inicial é parabólica, (f) a velocidade de permeação é determinada pelo modelo da resistência em série e (g) assume-se a camada de concentração como sendo homogênea e a equação de Carman-Kozeny é válida.

A solução numérica é obtida aplicando o método implícito de diferenças finitas. As derivadas das equações do escoamento foram aproximadas pelo esquema de diferença central com precisão de segunda ordem. A discretização destas equações conduz a um conjunto de equações algébricas lineares, que foi resolvido através do método de eliminação de Gauss.

A partir dos resultados numéricos, a concentração de polarização pode ser analisada em termos da espessura da camada limite de concentração. Estes resultados mostram que, para 
altos números de Reynolds axiais e também altos números de Schmidt, tem-se um decréscimo na espessura da camada limite de concentração local. Adicionalmente, a evolução da espessura da camada limite da concentração local, para um dado número de Reynolds na parede, depende dos valores do número de Schmidt e número de Reynolds axial (DAMAK et al., 2004).

No trabalho de Damak et al. (2004A) é utilizado o mesmo problema e a mesma modelagem analisada anteriormente em Damak et al. (2004), contudo a análise do problema se baseia nas seguintes hipóteses: (a) na entrada, a concentração de soluto é constante e igual à concentração do alimentador, (b) na saída, o fluxo desenvolvido e a baixa difusividade molecular do soluto permitem a aplicação de uma condição de um perfil de concentração desenvolvido, sem influência do escoamento acima do comprimento do tubo, (c) no eixo de simetria, não existe cruzamento de escoamento de massa e (d) na parede permeável, não existe acúmulo de partículas na superfície da membrana em regime permanente, isto é, todas as partículas são quase $100 \%$ rejeitadas pela membrana (concentração de filtrado aproximadamente zero).

De forma análoga à realizada em Damak et al. (2004), a solução numérica é obtida aplicando o método implícito de diferenças finitas. O modelo numérico desenvolvido aqui prediz com sucesso os mecanismos fundamentais envolvidos no comportamento do declínio do fluxo durante a filtração tangencial. O perfil de concentração axial destaca uma importante influência do comprimento da membrana. Os resultados apresentados mostram que a influência do número de Reynolds na parede é um importante parâmetro que controla a deposição de partículas sobre a superfície permeável da membrana.

O número de Reynolds na parede influencia o perfil de concentração de soluto e também controla a concentração polarizada em toda a extensão do tubo poroso. A 
concentração de polarização, sobre uma grande faixa de condições de operação, pode ser analisada em termos da espessura da camada limite de concentração. Uma correlação é mostrada envolvendo a espessura da camada do gel polarizado e os números de Reynolds axial, Reynolds na parede e Schmidt. Esta correlação mostra que o aumento do número de Reynolds conduz a uma baixa concentração de polarização, e para um baixo número de Schmidt, o aumento do número de Reynolds na parede não permite uma diminuição da concentração de polarização (DAMAK et al., 2004A).

O fenômeno de polarização da concentração, em regime estacionário, nos processos de filtração tangencial, pode ser descrito pelas equações de convecção e difusão, acopladas ao modelo da resistência por transporte de permeado (PARIS et al., 2002). O problema analisado é o fenômeno de transferência de massa em tubo poroso em regime de escoamento laminar, cujas coordenadas são (z,r, $\varphi$ ). A análise é baseada nas seguintes hipóteses: (a) a viscosidade e a densidade do fluido são constantes e iguais aos do solvente puro, (b) o coeficiente de difusão depende da concentração de soluto, (c) o fluido é incompressível e as condições do regime estacionário são controladas, (d) o movimento é considerado axisimétrico, assim independente de $\varphi$, (e) na entrada do tubo, o perfil de velocidade axial u(r,0) é parabólica e idêntica àquela obtida sem o escoamento de permeação, (f) dado que a taxa de escoamento tangencial (aproximadamente $10^{-6} \mathrm{~m}^{3} \mathrm{~s}^{-1}$ ) é muito maior que a do escoamento de permeado (aproximadamente $10^{-8} \mathrm{~m}^{3} \mathrm{~s}^{-1}$ ), consideraremos negligenciáveis a taxa de escoamento tangencial e a variação da velocidade axial ao longo do comprimento da membrana, (g) a difusão axial é negligenciada, (h) consideram-se a difusão radial e a convecção radial e (i) o perfil de pressão axial é bem conhecido e linear devido à diferença de pressão.

O modelo para o problema acima foi resolvido por meio do método de volumes finitos, usando malha não uniforme que foi refinada próximo à camada limite. Paris et al. (2002) 
concluem que os resultados experimentais não estão de acordo com o modelo do gel polarizador e do modelo da pressão osmótica: a concentração do soluto na superfície da membrana não é constante, mas depende da velocidade da corrente principal. A pressão osmótica calculada não é alta, se comparada à pressão transmembrana, para justificar o decréscimo do fluxo transmembrana. Depois de várias modificações, as resistências em séries modelaram os cálculos para a influência da resistência de polarização da concentração média de soluto na camada limite. Em dada velocidade, é obtida uma boa aceitação para toda a pressão utilizada. O modelo bidimensional baseado na resolução numérica da equação convectiva difusiva melhora a compreensão do fenômeno de transporte. Em baixas concentrações iniciais, os resultados não são muito satisfatórios. Em outros casos, a predição do escoamento de permeado tem uma boa aproximação com os resultados experimentais e a influência da velocidade, a concentração inicial, a pressão transmembrana e o comprimento da membrana (PARIS et al., 2002).

Hung e Perng (1991) investigam o desenvolvimento hidrodinâmico de um potencial relacionado a um fluido não - newtoniano em um tubo circular com injeção e sucção na parede. As equações de Navier-Stokes modificadas, para um potencial líquido, são diferentes das equações de Navier-Stokes convencionais em que a viscosidade do potencial líquido é dependente da taxa de cisalhamento, mas a viscosidade newtoniana é independente da taxa de cisalhamento. O problema considerado é um escoamento isotérmico, regime laminar e estacionário de um fluido não - newtoniano e incompressível na região de entrada de um tubo circular com parede porosa. $\mathrm{O}$ escoamento é bidimensional e uniforme na entrada do tubo. $\mathrm{O}$ fluido que é injetado é supostamente idêntico ao fluido escoando no tubo. A origem do sistema de coordenadas cilíndricas está na entrada do tubo. As equações de Navier-Stokes modificadas são resolvidas usando o processo de diferenças finitas e o procedimenteo de solução SIMPLER (Semi-Implicit Method for Pressure Linked Equations, Revised). A solução 
numérica para o escoamento proposto por Hung e Perng (1991) foi obtida através da solução das equações de Navier-Stokes modificadas.

Mignard e Glass (2001) investigam a impregnação da membrana durante o processo de ultrafiltração de solução de proteína. O modelo prediz o perfil de velocidade axial, o perfil de velocidade radial, a pressão, concentração em regime estacionário, a espessura local da proteína coagulada e a resistência de entupimento da camada coagulada. As equações de transporte acopladas para o momentum e soluto obedecem as seguintes condições de contorno: na entrada do tubo o perfil de velocidade axial é parabólico, na parede do tubo é utilizado o modelo da resistência em série. O entupimento foi predito somente como coagulação de proteína, quando as equações de difusividade e da continuidade divergiam. Para solução do modelo foi utilizado o método das diferenças finitas. Mignard e Glass (2001) concluem que o modelo predisse bem a resistência total do fluxo, mas a resistência da coagulação foi subestimada. Para melhorar a predição da resistência de coagulação, pode-se considerar a possibilidade de alta velocidade nos poros. O modelo apresentado pode ter bons valores práticos, contanto que uma descrição adequada do tamanho dos agregados seja conhecida.

\subsection{TÉCNICA DE TRANSFORMADA INTEGRAL GENERALIZADA (GITT)}

O desenvolvimento tecnológico promovido com o auxílio do computador levou ao aparecimento de técnicas puramente numéricas de solução, destacando-se os métodos de diferenças finitas e elementos finitos como ferramentas que possibilitam o processamento do cálculo numérico avançado através da utilização de linguagens de programação científicas. Genericamente, os métodos puramente numéricos baseiam-se na discretização temporal e espacial das equações originais e representativas de um problema físico. 
$\mathrm{Na}$ literatura, observa-se, de forma crescente, a partir da década de oitenta, certo renascimento das técnicas ditas analíticas, voltadas para um melhor aproveitamento dos recentes avanços em análise numérica, linguagens de programação e métodos computacionais associados a problemas da matemática avançada. Estes investimentos buscam diminuir o tempo de processamento, melhorar a precisão dos resultados e diminuir os esforços despendidos pelo programador nos estágios de construção e implementação algorítmicas.

Os métodos híbridos consistem de uma combinação de técnicas analíticas associadas às aproximações numéricas e surgiram como alternativa aos métodos puramente numéricos para a solução de problemas complicados de engenharia, os quais eram antes tratados apenas numericamente. Dentre os métodos híbridos, a GITT vem se tornando uma importante ferramenta na solução de problemas em convecção-difusão, com controle automático do erro global e acentuada redução no tempo de processamento, comparativamente aos métodos puramente numéricos. A GITT é uma técnica espectral, com natureza híbrida numéricoanalítica, e se baseia na Técnica de Transformada Integral Clássica (CITT) para soluções de equações diferenciais parciais lineares em difusão de calor e massa. A partir desta técnica, novos desenvolvimentos foram efetuados tornando-a mais flexível e aplicável aos problemas não-lineares mais complexos (PEREIRA, 2000).

No trabalho de Özisik (1968) encontram-se soluções de problemas de valor de contorno linear (condução de calor) com aplicação sistemática da técnica de transformada integral. Entre as várias diferentes aproximações existentes para a solução do problema de valor de contorno de condução de calor, a técnica da transformada integral é a que oferece uma direta e elegante aproximação, contanto que o par de transformadas e os núcleos estejam disponíveis (ÖZISIK, 1968).

A solução de problemas não-lineares mais complexos tornou-se possível sem muitas 
simplificações no seu modelo matemático original, graças à disponibilidade de pacotes computacionais comerciais capazes de resolverem sistemas de equações diferenciais ordinárias rígidos com procedimentos bem testados de controle de erro local. Associado a isto, o controle adaptativo das ordens de truncamento nas expansões em autofunções, resultantes da aplicação da transformada integral, fornece a esta técnica a característica de funcionamento com controle automático de erro, oferecendo ao usuário a possibilidade de trabalhar com uma precisão pré-estabelecida. Assim, a técnica pode ser utilizada como ferramenta no estabelecimento de resultados benchmark, bem como para co-validação de outros métodos numéricos aplicados para a solução de equações diferenciais parciais (PEREIRA, 2000).

Özisik e Murray publicaram em 1974 uma nova técnica de características analíticonuméricas para resolução de sistemas de equações diferenciais parciais, a princípio não tratáveis pela teoria clássica de separação de variáveis, a qual dispensava a necessidade do problema ser separável à priori. Estavam assim estabelecidos os formalismos básicos para o surgimento da denominada Técnica de Transformada Integral Clássica (CITT).

Em um estágio bem mais avançado, Mikhailov e Özisik lançam em 1984, o primeiro livro generalizando os formalismos da CITT (MIKHAILOV e ÖZISIK, 1984 apud CUNHA, 2002), que postulava um tratamento unificado, segundo sete classes de equações diferenciais parciais que foram definidas a partir de inúmeros problemas de transferência de calor e massa encontrados na literatura. A partir de então, convencionou-se renomear o método, sendo intitulado como GITT. Na década de oitenta, observa-se uma série de extensões da técnica de transformada integral clássica para resolução de diversos problemas abordados principalmente na literatura ocidental, que eram resolvidos por métodos discretizados denominados "puramente" numéricos, como diferenças finitas, elementos finitos, volumes finitos e suas 
variantes (SCOFANO NETO et al., 1989; APARECIDO e COTTA, 1987 apud CUNHA, 2002). Comparando-se as características da abordagem da CITT, com as técnicas citadas, observou-se o aparecimento de uma série de vantagens (COTTA, 1993; COTTA, 1994 apud CUNHA 2002): (a) metodologia sistemática de solução, (b) redução do tempo de processamento, (c) controle prescrito de erro, (d) aceleração da taxa de convergência numérica, (e) inexistência de malhas (fator que se acentua criticamente para problemas multidimensionais), (f) determinação numérica direta da função em um ponto (para valores definidos de tempo e espaço) sem necessidade de cálculo numérico de estados temporais anteriores ou de outros pontos do domínio espacial e (g) versatilidade do método em se tornar híbrido com outros, devido às suas características analítico-numéricas.

Em função do processo evolutivo encontrado no método de transformada integral, em 1993, Cotta publicou o segundo livro relativo ao assunto, apresentando uma revisão dos formalismos clássicos, que são agora estendidos com ênfase para a solução de problemas nãolineares e fortemente acoplados e propondo técnicas para melhorar a eficiência da solução numérica.

A natureza da GITT é híbrida numérico-analítica, assim o tempo de processamento dispensado à tarefa numérica envolvida na solução é sempre reduzido a uma única variável independente, sendo a solução final descrita analiticamente, em forma explícita, nas demais variáveis independentes. Assim, o aumento do tempo de processamento é apenas moderado ao se incrementar o número de dimensões do problema considerado, o que torna esta metodologia bastante atraente para o tratamento de problemas multidimensionais. Aliado a isto, a utilização de filtros analíticos, a escolha de bases mais representativas do problema original e a utilização de plataforma de manipulação simbólica (COTTA e MIKHAILOV, 1997), contribuem de forma positiva no aperfeiçoamento desta técnica (PEREIRA, 2000). 
Genericamente, para solução de um problema segundo o formalismo da GITT, necessita-se de um par transformada-inversa e de um problema auxiliar associado, que incorpore características analíticas, físicas e de condição de contorno do problema original. Os problemas originais são equações diferenciais parciais, juntamente com as condições de contorno, condição de entrada e de saída, que modelam determinado processo físico. A eliminação de variáveis independentes, por meio de operadores de integração apropriados, leva à obtenção de um sistema de equações diferenciais ordinárias, que é denominado de sistema transformado. Num passo seguinte, este último é truncado de uma ordem finita e prescrita, N, para ser resolvido analítica e numericamente. Caso o sistema transformado possa apresentar solução analítica, esta pode ser obtida automaticamente.

Do ponto de vista das aplicações práticas de engenharia, pode-se citar o sucesso da utilização da GITT na análise de equipamentos termohidráulicos, migração de rejeitos radioativos em solos, aerotermodinâmica de veículos espaciais, poluição ambiental, processos de secagem, problemas térmicos em siderurgia, enriquecimento isotópico, combustão, resfriamento de equipamentos eletrônicos, reservatórios de petróleo, entre outros. Todos esses problemas práticos de engenharia foram ou continuam sendo investigados, no âmbito de projetos firmados entre a COPPE, através do Laboratório de Transmissão e Tecnologia do Calor (LTTC), e outras instituições públicas e privadas.

Atualmente, os problemas não-lineares de convecção-difusão têm recebido uma maior atenção dos grupos de pesquisa envolvidos com o uso desta técnica, por constituírem uma grande parcela dos problemas práticos de engenharia térmica.

Para a utilização da GITT alguns passos devem ser aplicados sequencialmente, os quais podem ser assim resumidos: 
i. Escolha de um problema auxiliar apropriado (Apêndice (2)), o qual deve conter o máximo de informações possível sobre o problema original, no que diz respeito à geometria e operadores, nas coordenadas a serem eliminadas pela transformação integral;

ii. Obtenção do par transformada-inversa (Apêndice (3)), a partir da propriedade de ortogonalidade das autofunções para transformação do problema e posterior inversão dos campos transformados obtidos;

iii. Transformação integral do sistema diferencial parcial original, resultando em um sistema infinito e acoplado de equações diferenciais ordinárias;

iv. Truncamento desse sistema diferencial ordinário resultante e obtenção de sua solução numérica;

v. Obtenção dos potenciais originais utilizando a formula de inversão.

A seguir, veremos alguns trabalhos envolvendo modelos matemáticos, tratados pela literatura, e relacionados à GITT, onde a partir dos mesmos procurou-se expor o seguinte: (a) o problema a ser modelado, (b) modelagem, (c) desenvolvimento da GITT e (d) conclusão.

O desenvolvimento simultâneo da distribuição de velocidade e de temperatura de um escoamento laminar dentro de um canal plano e paralelo foi analiticamente estudado no trabalho de Silva, Cotta e Aparecido (1990). Para isto, foi adotado um processo de linearização para o problema da velocidade e resolvenda a equação de energia desacoplada através da GITT. Uma solução completa é obtida dentro de um grande intervalo para a coordenada axial, provinda de avaliação numérica do sistema transformado de equações diferenciais ordinárias. Neste sentido, soluções explícitas aproximadas são obtidas para estimativas rápidas no contexto da aplicação. São investigados vários aspectos, tais como a 
influência da convecção transversal, os efeitos de diferentes perfis de velocidade, convergência de soluções completas e precisão de soluções aproximadas.

A convecção forçada laminar dentro de um duto retangular foi estudada analiticamente utilizando a GITT (APARECIDO e COTTA, 1990), permitindo a solução de problemas convectivos-difusivos com problemas de autovalores não-separáveis. Resultados de referência são estabelecidos para quantidades de interesse prático dentro da região de entrada térmica, para um grande intervalo da variável axial. Para ambas, as regiões em desenvolvimento e completamente desenvolvidas, é feita a análise crítica da precisão de resultados obtidos de aproximações numéricas.

Resultados de referência (benchmark), para um escoamento em torno de degrau (backward-facing step) especifico, são obtidos pela GITT. Esta aproximação numéricoanalítica híbrida é empregada para controlar as equações de Navier-Stokes bidimensionais na formulação de funções de corrente. Resultados numéricos com controle de precisão global automático foram produzidos para diversos valores do número de Reynolds. Foram realizadas comparações críticas com resultados experimentais previamente informados com excelentes aproximações. Também são validadas algumas diferentes aproximações puramente numéricas (PÉREZ-GUERRERO e COTTA, 1996).

Cotta e Pimentel (1998) modelaram um escoamento turbulento de um fluido newtoniano e incompressível, desenvolvido entre placas paralelas, cuja distribuição de velocidade de entrada é uniforme e com uma taxa de validade para a formulação da camada limite turbulento. Para este referido problema, utilizaram a GITT para simular e obtenção de resultados. O modelo algébrico de Cebeci-Schmith foi escolhido para prover as requeridas equações para difusão turbulenta. Foi demonstrado o comportamento da convergência de expansões de autofunções e também foi discutida a escolha de diferentes problemas de 
autovalor. Resultados numéricos do perfil de velocidade que convergiram completamente foram comparados com resultados experimentais e com outros resultados da literatura que se relacionam ao problema turbulento.

No trabalho de Silva, Pérez-Guerrero e Cotta (1999) as equações da camada limite para um escoamento laminar incompressível e estacionário em canal foram resolvidas através da GITT, adotando a formulação de função de corrente. Esta aproximação híbrida analíticonumérica proporciona resultados de referência (benchmark) com precisão prescrita pelo usuário e foi utilizada na validação de esquemas puramente numéricos. Os relativos méritos da formulação de função de corrente foram ilustrados através de resultados numéricos para o comportamento da convergência no caso do escoamento plano de Poiseuille.

Utilizando a formulação de função de corrente e a GITT, foi estudado no trabalho de Luz Neto, Quaresma e Cotta (2002) o modelo de Darcy tridimensional e transiente de convecção natural em cavidade preenchida por meios porosos. Foi desenvolvida uma formulação geral e uma metodologia de solução para uma cavidade vertical (parede vertical separada e com temperaturas na parede horizontal diferencial). Foram apresentados resultados por cavidades cúbicas e também avaliado o efeito do número de Rayleigh para situações estáveis, observando a evolução transiente do processo de transferência de calor. O comportamento da convergência da solução de expansão de autofunções é investigado e foram feitas comparações com resultados da literatura.

No trabalho de Magno, Macedo e Quaresma (2002) a GITT é empregada na solução das equações de camada limite simultâneas e relacionada ao escoamento laminar em desenvolvimento e ao fluido não-newtoniano dentro de um canal de placas planas. Nesta modelagem, as equações de momentum e energia dentro de um intervalo de validade das equações de camada limite, a formulação da função de corrente é empregada, pois esta 
oferece um melhor desempenho computacional se comparado à formulação das variáveis primitivas. Resultados numéricos para a temperatura central e número de Nusselt são estabelecidos ao longo da posição axial do canal e para vários índices de potêncial. Com estes resultados são feitas comparações críticas com trabalhos da literatura.

A GITT foi estendida para controlar as equações de Navier-Stokes incompressíveis para um escoamento laminar estacionário bidimensional em geometria cilíndrica (PEREIRA, PÉREZ GUERRERO e COTTA, 1998). Foram obtidas soluções híbridas analítico-numéricas com controle de precisão. Tais soluções são resultados de uma escolha aproximada de bases de expansões de autofunções associadas, extraídos do operador de difusão da formulação de função corrente para esta classe de problemas. A aproximação é ilustrada para um escoamento laminar em desenvolvimento dentro de um canal anular e resultados numéricos foram obtidos para demonstrar a excelente convergência, que é característica dos métodos híbridos. Comparações foram feitas para a formulação da camada limite e um conjunto de resultados de referência (benchmark) foram produzidos, para diferentes valores de Reynolds. 


\section{Formulação Matemática e}

\section{Resoluções Via GITT}

Neste capítulo temos duas formulações matemáticas para o problema de transporte de massa por convecção, as quais serão solucionadas via GITT. Nas Seções 3.1 e 3.2 temos a formulação matemática e desenvolvimento da GITT, respectivamente, para a equação convectiva-difusiva elíptica e a equação convectiva-difusiva parabólica. Uma formulação da espessura da camada limite de concentração, bem como uma metodologia (ou critério) para determinação da taxa assintótica, será exposto na Seção (3.3). As análises dos resultados provindos destas modelagens serão vistos no Capítulo 4, Resultados e Discussões.

\subsection{EQuaÇão Convectiva - DifuSiva ElíPtica}

\subsubsection{Formulação e Modelagem Matemática}

O problema estabelecido nesta seção é de um escoamento de um fluido em regime laminar em tubo com superfície permeável, de comprimento " $L$ " e raio " $\mathrm{R}_{0}$ ", cujas coordenadas em geometria cilíndrica adimensionais são " $X$ " e " $R$ ". 
A Figura 8 ilustra os principais elementos do escoamento num tubo permeável essencialmente representativo do processo de filtração tangencial. Mostra também as camadas limites hidrodinâmica e de concentração. Próximo à superfície permeável interna, por um lado, vemos a camada de concentração na parede e por outro o fluxo transmembrana.

As hipóteses apresentadas nesta modelagem são as seguintes:

i. A densidade e viscosidade do fluido são consideradas constantes;

ii. O coeficiente de difusão do soluto é considerado constante;

iii. Escoamento em regime permanente;

iv. O movimento é considerado axisimétrico;

v. Na entrada do tubo, a concentração, $\mathrm{c}_{0}$, é considerada uniforme;

vi. A velocidade de entrada é considerada parabólica;

vii. A velocidade de permeação, $\mathrm{v}_{\mathrm{w}}$, é considerada uniforme;

viii. O campo gravitacional é negligenciado;

ix. Os perfis de velocidades radial e axial, $V \equiv V(R)$ e $U \equiv U(X, R)$, respectivamente, são dados pelas Equações (34), (35) e (36);

x. A concentração na parede, $\mathrm{c}_{\mathrm{w}}$, é dada pela Equação (18). 


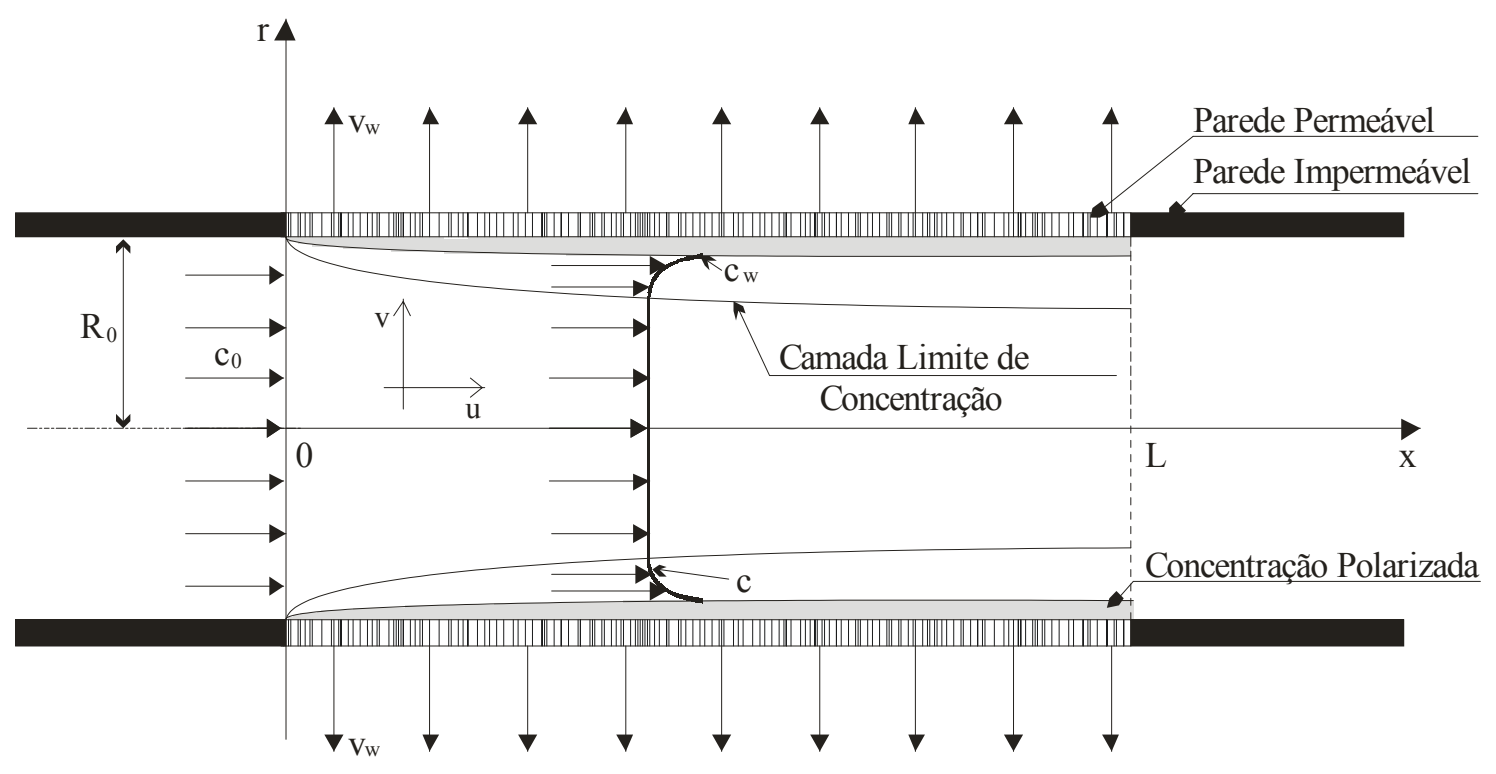

Figura 8. Representação esquemática do perfil de concentração em tubo permeável de raio $\mathrm{R}_{0}$, cuja concentração de entrada é $\mathrm{c}_{0}$.

Com estas suposições, investigaremos um fluido newtoniano e incompressível em tubo com superfície permeável, modelado a partir da equação de conservação das espécies químicas (equação convectiva-difusiva elíptica), Equação (19), e as condições de contorno, de entrada e de saída, respectivamente, Equações: (22), (23), (24) e (25), as quais serão escritas, na forma adimensional, como:

$$
\mathrm{V} \frac{\partial \mathrm{C}}{\partial \mathrm{R}}+\mathrm{U} \frac{\partial \mathrm{C}}{\partial \mathrm{X}}=\frac{1}{\mathrm{Pe}}\left[\frac{1}{\mathrm{R}} \frac{\partial}{\partial \mathrm{R}}\left(\mathrm{R} \frac{\partial \mathrm{C}}{\partial \mathrm{R}}\right)+\frac{\partial^{2} \mathrm{C}}{\partial \mathrm{X}^{2}}\right]
$$

sendo $\mathrm{C} \equiv \mathrm{C}(\mathrm{X}, \mathrm{R}), 0 \leq \mathrm{R} \leq 1,0 \leq \mathrm{X} \leq \xi, \xi=\mathrm{L} / \mathrm{R}_{0}$ :

- no centro do tubo, $\mathrm{R}=0,0 \leq \mathrm{X} \leq \xi$ :

$$
\mathrm{C}(\mathrm{X}, 0)=1
$$

- na parede do tubo, $\mathrm{R}=1,0 \leq \mathrm{X} \leq \xi$ :

$$
C(X, 1)=C_{w}(X)=\frac{1}{1-\frac{v_{w}}{D} \delta(X)}
$$


- na entrada do tubo, $\mathrm{X}=0,0 \leq \mathrm{R} \leq 1$ :

$$
\mathrm{C}(0, \mathrm{R})=1
$$

- na saída do tubo, $\mathrm{X}=\xi, 0 \leq \mathrm{R} \leq 1$ :

$$
\left.\frac{\partial \mathrm{C}(\mathrm{X}, \mathrm{R})}{\partial \mathrm{X}}\right|_{\mathrm{X}=\xi}=0
$$

\subsubsection{DESENVOLVIMENTO DA GITT}

Para resolvermos o problema original caracterizado pelas Equações (48) até (52), utilizaremos a GITT (COTTA, 1993), cujas principais etapas podem ser encontradas na Seção (2.3).

Devido a condições de contorno, dadas pelas Equações (50) e (51), serem não homogêneas, utilizaremos um filtro, $\mathrm{F}(\mathrm{R} ; \mathrm{X})$, com a intenção de reescrever o problema original em outro, com condições de contorno homogêneas:

$$
\mathrm{C}(\mathrm{X}, \mathrm{R})=\mathrm{C}_{\mathrm{H}}(\mathrm{X}, \mathrm{R})+\mathrm{F}(\mathrm{R} ; \mathrm{X})
$$

A função "F" representa o perfil de concentração para um escoamento completamente desenvolvido e sua expressão matemática, bem como a condição de contorno, pode ser encontrada no Apêndice (1). $O$ potencial $C_{H}$ representa a concentração com condição de contorno homogênea na direção axial.

Substituindo a Equação (53) na Equação (48) e também nas condições de contorno e entrada, Equações. (49) até (51), obtemos: 


$$
\begin{aligned}
\mathrm{U} \frac{\partial \mathrm{C}_{\mathrm{H}}}{\partial \mathrm{X}}+\mathrm{V} \frac{\partial \mathrm{C}_{\mathrm{H}}}{\partial \mathrm{R}}= & \frac{1}{\text { Pe R }} \frac{\partial}{\partial \mathrm{R}}\left(\mathrm{R} \frac{\partial \mathrm{C}_{\mathrm{H}}}{\partial \mathrm{R}}\right)+\frac{1}{\mathrm{Pe}} \frac{\partial^{2} \mathrm{C}_{\mathrm{H}}}{\partial \mathrm{X}^{2}} \\
& +\frac{1}{\mathrm{Pe}} \frac{\partial^{2} \mathrm{~F}}{\partial \mathrm{X}^{2}}-\mathrm{U} \frac{\partial \mathrm{F}}{\partial \mathrm{X}}-\mathrm{V} \frac{\partial \mathrm{F}}{\partial \mathrm{R}}
\end{aligned}
$$

sendo $\mathrm{C}_{\mathrm{H}} \equiv \mathrm{C}_{\mathrm{H}}(\mathrm{X}, \mathrm{R}), 0 \leq \mathrm{X} \leq \xi$ e $0 \leq \mathrm{R} \leq 1$ e:

$$
\begin{aligned}
& \mathrm{C}_{\mathrm{H}}(\mathrm{X}, 0)=0 \\
& \mathrm{C}_{\mathrm{H}}(\mathrm{X}, 1)=0 \\
& \mathrm{C}_{\mathrm{H}}(0, \mathrm{R})=1-\mathrm{F}(\mathrm{R} ; 0)=0 \\
& \left.\frac{\partial \mathrm{C}_{\mathrm{H}}(\mathrm{X}, \mathrm{R})}{\partial \mathrm{X}}\right|_{\mathrm{X}=\xi}=\left.\left(\frac{\ln (\mathrm{R})}{\ln \left(\mathrm{R}_{\min }\right)}-1\right) \frac{\mathrm{dC}_{\mathrm{w}}(\mathrm{X})}{\mathrm{dX}}\right|_{\mathrm{X}=\xi}
\end{aligned}
$$

O problema descrito pelas Equações (54) até (58) agora têm as condições de contorno (Equações (55) e (56)) homogêneas e está passível de uso da GITT.

No Apêndice (2) mostramos como obter o problema auxiliar (ou problema de autovalor), o qual é um problema do tipo Sturm-Liouville. Seguindo a técnica, o problema auxiliar apropriado é tomado como:

$$
\frac{\mathrm{d}^{2} \psi_{\mathrm{i}}(\mathrm{R})}{\mathrm{dR}^{2}}+\frac{1}{\mathrm{R}} \frac{\mathrm{d} \psi_{\mathrm{i}}(\mathrm{R})}{\mathrm{dR}}+\beta_{\mathrm{i}}{ }^{2} \psi_{\mathrm{i}}(\mathrm{R})=0, \quad \mathrm{i}=1,2,3 \ldots \text { e } \mathrm{R}_{\min } \leq \mathrm{R} \leq 1
$$

com as seguintes condições de contorno:

$$
\begin{aligned}
& \psi_{\mathrm{i}}\left(\mathrm{R}_{\text {min }}\right)=0 \\
& \psi_{\mathrm{i}}(1)=0
\end{aligned}
$$

A expressão dada pela Equação (59) é a conhecida equação diferencial de Bessel de ordem zero, onde $\beta_{\mathrm{i}}$ e $\psi_{\mathrm{i}}$ são, respectivamente, os autovalores e autofunções. As autofunções são obtidas por (ÖZISIK, 1980):

$$
\psi_{\mathrm{i}}(\mathrm{R})=\mathrm{J}_{0}\left(\beta_{\mathrm{i}} \mathrm{R}\right) \mathrm{Y}_{0}\left(\beta_{\mathrm{i}}\right)-\mathrm{J}_{0}\left(\beta_{\mathrm{i}}\right) \mathrm{Y}_{0}\left(\beta_{\mathrm{i}} \mathrm{R}\right)
$$


para $\mathrm{R}_{\min } \leq \mathrm{R} \leq 1$, enquanto que os autovalores são obtidos da equação:

$$
\mathrm{J}_{0}\left(\beta_{\mathrm{i}} \mathrm{R}_{\text {min }}\right) \mathrm{Y}_{0}\left(\beta_{\mathrm{i}}\right)-\mathrm{J}_{0}\left(\beta_{\mathrm{i}}\right) \mathrm{Y}_{0}\left(\beta_{\mathrm{i}} \mathrm{R}_{\text {min }}\right)=0
$$

onde $\mathrm{J}_{0}$ e $\mathrm{Y}_{0}$ são as funções de Bessel de ordem zero de primeira e segunda classe, respectivamente (ÖZISIK, 1980).

As Equações de (59) até (61) (ver Apêndice (3)) satisfazem a seguinte propriedade de ortogonalidade (COTTA, 1993):

$$
\mathrm{N}_{\mathrm{ij}}=\int_{\mathrm{R}_{\min }}^{1} \mathrm{R} \psi_{\mathrm{i}} \psi_{\mathrm{j}} \mathrm{dR}=\left\{\begin{array}{cc}
0, & \mathrm{i} \neq \mathrm{j} \\
\mathrm{N}_{\mathrm{i}}, & \mathrm{i}=\mathrm{j}
\end{array}, \quad \mathrm{i}, \mathrm{j}=1,2,3 \ldots\right.
$$

As autofunções normalizadas são definidas por:

$$
\tilde{\psi}_{\mathrm{i}}(\mathrm{R})=\frac{\psi_{\mathrm{i}}(\mathrm{R})}{\mathrm{N}_{\mathrm{i}}^{1 / 2}}
$$

sendo a integral de normalização dada por (ÖZISIK, 1980):

$$
\mathrm{N}_{\mathrm{i}}=\frac{2}{\pi^{2}} \frac{\mathrm{J}_{0}^{2}\left(\beta_{\mathrm{i}} \mathrm{R}_{\min }\right)-\mathrm{J}_{0}^{2}\left(\beta_{\mathrm{i}}\right)}{\beta_{\mathrm{i}}^{2} \mathrm{~J}_{0}^{2}\left(\beta_{\mathrm{i}} \mathrm{R}_{\min }\right)}
$$

O problema de autovalor resolvido acima nos permite definir o seguinte par transformada - inversa (Apêndice (3)):

$$
\begin{array}{ll}
\bar{C}_{i}(X)=\int_{R_{\min }}^{1} \mathrm{R} \tilde{\psi}_{\mathrm{i}}(\mathrm{R}) \mathrm{C}_{\mathrm{H}}(\mathrm{X}, \mathrm{R}) \mathrm{dR}, & \text { Transformada } \\
\mathrm{C}_{\mathrm{H}}(\mathrm{X}, \mathrm{R})=\sum_{\mathrm{i}=1}^{\infty} \tilde{\psi}_{\mathrm{i}}(\mathrm{R}) \overline{\mathrm{C}}_{\mathrm{i}}(\mathrm{X}), & \text { Inversa }
\end{array}
$$

O processo de transformada integral é executado no sistema dado pelas Equações (54) até (57), assim, na Equação (54), aplicamos o operador $\int_{\mathrm{R}_{\min }}^{1} \mathrm{R} \tilde{\psi}_{\mathrm{i}}(\mathrm{R}) \mathrm{dR}$ e depois de usarmos a fórmula de inversão (Equação (68)), obtemos: 


$$
\frac{1}{\operatorname{Pe}} \sum_{j=1}^{\infty} \delta_{i j} \frac{d^{2} \bar{C}_{j}}{d X^{2}}=U_{2} \sum_{j=1}^{\infty} \mathrm{Al}_{i j} \frac{d \bar{C}_{j}}{d X}+\sum_{j=1}^{\infty} \mathrm{Q}_{i j} \bar{C}_{j}+H_{i}, \quad i=1,2,3 \ldots
$$

com:

$$
\begin{aligned}
\mathrm{Q}_{\mathrm{ij}}= & \mathrm{A} 2_{\mathrm{ij}}+\frac{1}{\mathrm{Pe}} \beta_{\mathrm{j}}^{2} \delta_{\mathrm{ij}} \\
\mathrm{H}_{\mathrm{i}}= & -\frac{1}{\operatorname{Pe}} \frac{\mathrm{d}^{2} \mathrm{C}_{\mathrm{w}}}{\mathrm{dX}} \mathrm{B} 1_{\mathrm{i}}+\frac{1}{\operatorname{Pe} \ln \left(\mathrm{R}_{\text {min }}\right)} \frac{\mathrm{d}^{2} \mathrm{C}_{\mathrm{w}}}{\mathrm{dX} \mathrm{X}^{2}} 2_{\mathrm{i}}+\mathrm{U}_{2} \frac{\mathrm{dC} \mathrm{w}_{\mathrm{w}}}{\mathrm{dX}} \mathrm{B} 3_{\mathrm{i}}- \\
& -\frac{\mathrm{U}_{2}}{\ln \left(\mathrm{R}_{\text {min }}\right)} \frac{\mathrm{dC}_{\mathrm{w}}}{\mathrm{dX}} \mathrm{B} 4_{\mathrm{i}}+\frac{1-\mathrm{C}_{\mathrm{w}}}{\ln \left(\mathrm{R}_{\text {min }}\right)} \mathrm{B} 5_{\mathrm{i}}
\end{aligned}
$$

Os coeficientes relacionados são determinados pelas seguintes integrais:

$$
\begin{aligned}
& \mathrm{Al}_{\mathrm{ij}}=\int_{\mathrm{R}_{\min }}^{1} \mathrm{R} \mathrm{U}_{1} \tilde{\Psi}_{\mathrm{i}} \tilde{\psi}_{\mathrm{j}} \mathrm{dR} \\
& \mathrm{A} 2_{\mathrm{ij}}=\int_{\mathrm{R}_{\min }}^{1} \mathrm{RV} \tilde{\psi}_{\mathrm{i}} \tilde{\Psi}_{\mathrm{j}}^{\prime} \mathrm{dR} \\
& \mathrm{B} 1_{\mathrm{i}}=\int_{\mathrm{R}_{\min }}^{1} \mathrm{R} \tilde{\psi}_{\mathrm{i}} \mathrm{dR} \\
& \mathrm{B} 2_{\mathrm{i}}=\int_{\mathrm{R}_{\min }}^{1} \mathrm{R} \ln (\mathrm{R}) \tilde{\Psi}_{\mathrm{i}} \mathrm{dR} \\
& \mathrm{B} 3_{\mathrm{i}}=\int_{\mathrm{R}_{\min }}^{1} \mathrm{R} U_{1} \tilde{\Psi}_{\mathrm{i}} \mathrm{dR} \\
& \mathrm{B} 4_{\mathrm{i}}=\int_{\mathrm{R}_{\min }}^{1} \mathrm{R} \ln (\mathrm{R}) \mathrm{U}_{1} \tilde{\Psi}_{\mathrm{i}} \mathrm{dR} \\
& \mathrm{B} 5_{\mathrm{i}}=\int_{\mathrm{R}_{\min }}^{1} \mathrm{~V} \tilde{\Psi}_{\mathrm{i}} \mathrm{dR}
\end{aligned}
$$

A transformação integral (Equação (67)) produz a seguinte condição de contorno para o sistema dado pela Equação (69):

$$
\overline{\mathrm{C}}_{\mathrm{i}}(0)=\int_{\mathrm{R}_{\min }}^{1} \mathrm{R} \tilde{\psi}_{\mathrm{i}} \mathrm{C}_{\mathrm{H}}(0, \mathrm{R}) \mathrm{dR}=0
$$




$$
\frac{\mathrm{d} \bar{C}_{\mathrm{i}}}{\mathrm{dX}}(\xi)=\left.\int_{\mathrm{R}_{\min }}^{1} \mathrm{R} \tilde{\psi}_{\mathrm{i}} \frac{\mathrm{dC}_{\mathrm{H}}(\mathrm{X}, \mathrm{R})}{\mathrm{dX}}\right|_{\mathrm{X}=\xi} \mathrm{dR}=\left.\frac{1}{\ln \left(\mathrm{R}_{\min }\right)} \frac{\mathrm{dC}_{\mathrm{w}}}{\mathrm{dX}}\right|_{\mathrm{X}=\xi} \mathrm{B} 2_{\mathrm{i}}-\left.\frac{\mathrm{dC}_{\mathrm{w}}}{\mathrm{dX}}\right|_{\mathrm{X}=\xi} \mathrm{B} 1_{\mathrm{i}}
$$

A Equação (69) forma um sistema infinito de equações diferenciais ordinárias lineares (ou problema de valor de contorno), o qual pode ser truncado para ordens "N" suficientemente grandes para se calcular os potenciais " $\bar{C}_{i}(X)$ ". Na solução de tal sistema, emprega-se a sub-rotina DBVPFD do pacote IMSL (1991). Uma vez que os potenciais " $\overline{\mathrm{C}}_{\mathrm{i}}$ " tenham sido avaliados numericamente ao longo da coordenada axial "X", a transformada inversa $\left(C_{H}(X, R)=\sum_{i=1}^{N} \tilde{\psi}_{i}(R) \bar{C}_{i}(X)\right.$, Equação (68) com o devido truncamento) determina o potencial " $\mathrm{C}_{\mathrm{H}}(\mathrm{X}, \mathrm{R})$ ”, em qualquer posição de interesse e a Equação $\left(\mathrm{C}(\mathrm{X}, \mathrm{R})=\mathrm{C}_{\mathrm{H}}(\mathrm{X}, \mathrm{R})+\mathrm{F}(\mathrm{R} ; \mathrm{X})\right)$ completa a solução para a concentração “ $\mathrm{C}(\mathrm{X}, \mathrm{R})$ ”.

\subsection{EquaÇão Convectiva - Difusiva Parabólica}

\subsubsection{FormulaÇÃo E Modelagem MATEMÁtica}

A equação convectiva-difusiva parabólica, Equação (21), em coordenadas cilíndricas, é escrita na forma adimensional como:

$$
\mathrm{V} \frac{\partial \mathrm{C}}{\partial \mathrm{R}}+\mathrm{U} \frac{\partial \mathrm{C}}{\partial \mathrm{X}}=\frac{1}{\operatorname{Pe} \mathrm{R}} \frac{\partial}{\partial \mathrm{R}}\left(\mathrm{R} \frac{\partial \mathrm{C}}{\partial \mathrm{R}}\right)
$$

sendo $0 \leq \mathrm{X} \leq \mathrm{L} / \mathrm{R}_{0}, 0<\mathrm{R} \leq 1, \mathrm{C} \equiv \mathrm{C}(\mathrm{X}, \mathrm{R}), 0 \leq \mathrm{R} \leq 1,0 \leq \mathrm{X} \leq \xi$ e $\xi=\mathrm{L} / \mathrm{R}_{0}$. Os perfis de velocidade radial $U \equiv \mathrm{U}(\mathrm{X}, \mathrm{R})$, e axial, $\mathrm{V} \equiv \mathrm{V}(\mathrm{R})$, são dados pelas Equações (34), (35) e (36). As condições de contorno e de entrada são as mesmas das Equações (49), (50) e (51). Todas as hipóteses utilizadas na Seção (3.1.1) serão consideradas nesta modelagem. O desenvolvimento e alguns resultados, para o perfil de concentração e fluxo, desta seção também podem ser vistos no trabalho de Venezuela, Pérez-Guerrero e Fontes (2008, in press). 


\subsubsection{DESENVOLVIMENTO DA GITT}

Analogamente ao que fizemos na Seção (3.1.2), substituímos o filtro $\mathrm{C}(\mathrm{X}, \mathrm{R})=\mathrm{C}_{\mathrm{H}}(\mathrm{X}, \mathrm{R})+\mathrm{F}(\mathrm{R} ; \mathrm{X})$ (Equação (53)) no problema original, Equação (81), e obtemos:

$$
\mathrm{U} \frac{\partial \mathrm{C}_{\mathrm{H}}}{\partial \mathrm{X}}+\mathrm{V} \frac{\partial \mathrm{C}_{\mathrm{H}}}{\partial \mathrm{R}}=\frac{1}{\mathrm{Pe} \mathrm{R}} \frac{\partial}{\partial \mathrm{R}}\left(\mathrm{R} \frac{\partial \mathrm{C}_{\mathrm{H}}}{\partial \mathrm{R}}\right)-\mathrm{U} \frac{\partial \mathrm{F}}{\partial \mathrm{X}}-\mathrm{V} \frac{\partial \mathrm{F}}{\partial \mathrm{R}}
$$

sendo $0 \leq X \leq \xi, \quad 0 \leq R \leq 1, C_{H}(X, 0)=0, C_{H}(X, 1)=0, C_{H}(0, R)=0$ e " $F "$ dado no Apêndice (1).

Estabelecido o problema de autovalor, bem como sua solução (Apêndice (2)), e também o par de transformadas (analogamente ao que fizemos na Seção (3.1.2) e Apêndice (3)), obtemos o seguinte problema de valor inicial:

$$
\begin{aligned}
& \sum_{j=1}^{\infty} A_{i j} U_{2} \frac{d \bar{C}_{j}}{d X}=-\sum_{j=1}^{\infty}\left(B_{i j}+\frac{\beta_{j}^{2}}{P e} \delta_{i j}\right) \bar{C}_{j}-U_{2} \frac{d}{d Z}\left(\frac{1-C_{w}(X)}{\ln \left(R_{\text {min }}\right)}\right) B 1_{i}- \\
& -U_{2} \frac{d C_{w}(X)}{d X} B 2_{i}-\left(\frac{1-C_{w}(X)}{\ln \left(R_{\text {min }}\right)}\right) B 3_{i}, \quad i=1,2,3 \ldots
\end{aligned}
$$

Os coeficientes relacionados são determinados analiticamente pelas seguintes integrais:

$$
\begin{aligned}
& \mathrm{A}_{\mathrm{ij}}=\int_{\mathrm{R}_{\min }}^{1} \mathrm{RU}_{1} \tilde{\Psi}_{\mathrm{i}} \tilde{\Psi}_{\mathrm{j}} \mathrm{dR} \\
& \mathrm{B}_{\mathrm{ij}}=\int_{\mathrm{R}_{\min }}^{1} \mathrm{RV} \tilde{\psi}_{\mathrm{i}} \tilde{\psi}_{\mathrm{j}}^{\prime} \mathrm{dR} \\
& B 1_{i}=\int_{R_{\min }}^{1} R U_{1} \tilde{\Psi}_{i} d R \\
& \mathrm{~B} 2_{\mathrm{i}}=\int_{\mathrm{R}_{\min }}^{1} \mathrm{RU}_{1} \tilde{\psi}_{\mathrm{i}} \ln (\mathrm{R}) \mathrm{dR} \\
& B 3_{i}=\int_{R_{\min }}^{1} \mathrm{~V} \tilde{\psi}_{\mathrm{i}} \mathrm{dR}
\end{aligned}
$$


A transformação integral da condição de entrada (Equação (67)) produz a seguinte condição inicial para o sistema dado pela Equação (83):

$$
\overline{\mathrm{C}}_{\mathrm{i}}(0)=\int_{\mathrm{R}_{\min }}^{1} \mathrm{R} \tilde{\psi}_{\mathrm{i}} \mathrm{C}_{\mathrm{H}}(0, \mathrm{R}) \mathrm{dR}=0
$$

A Equação (83) forma um sistema infinito de equações diferenciais ordinárias lineares (ou problema de valor inicial), o qual pode ser truncado para ordens "N" suficientemente grandes, para se calcular os potenciais “ $\overline{\mathrm{C}}_{\mathrm{i}}(\mathrm{X})$ ". Na solução de tal sistema, emprega-se a subrotina DVIPAG do pacote IMSL (1991). Uma vez que os potenciais “ $\overline{\mathrm{C}}_{\mathrm{i}}$ ” tenham sido avaliados numericamente ao longo da coordenada axial "X", a transformada inversa $\left(C_{H}(X, R)=\sum_{i=1}^{N} \tilde{\psi}_{i}(R) \bar{C}_{i}(X)\right.$ Equação (68) com o devido truncamento) determina o potencial " $\mathrm{C}_{\mathrm{H}}(\mathrm{X}, \mathrm{R})$ ”, em qualquer posição de interesse e a Equação (53) $\left(\mathrm{C}(\mathrm{X}, \mathrm{R})=\mathrm{C}_{\mathrm{H}}(\mathrm{X}, \mathrm{R})+\mathrm{F}(\mathrm{R} ; \mathrm{X})\right)$ completa a solução para a concentração “ $\mathrm{C}(\mathrm{X}, \mathrm{R})$ ”.

\subsection{FormulaÇão da Espessura da Camada limite de ConcentraÇão e Concentração Polarizada}

\subsubsection{FormulaÇão Matemática}

A condição de contorno na superfície permeável $\mathrm{c}\left(\mathrm{x}, \mathrm{R}_{0}\right)=\mathrm{c}_{\mathrm{w}}(\mathrm{x}), \quad 0 \leq \mathrm{x} \leq \mathrm{L}$ (Equação (24)), exige uma expressão para o perfil de concentração na parede, e para tal utilizaremos a Equação $(18)\left(\mathrm{c}_{\mathrm{w}}(\mathrm{x})=\mathrm{c}_{0} / 1-\frac{\delta(\mathrm{x})}{\mathrm{D}} \mathrm{v}_{\mathrm{w}}\right)$. Esta exigência se evidencia ainda mais pelo uso da GITT, por exemplo, no cálculo da matriz coluna $\mathrm{H}_{\mathrm{i}}$ (Equação (71)) e da condição de fronteira $\mathrm{d} \overline{\mathrm{C}}_{\mathrm{i}}(\xi) / \mathrm{dX}$ (Equação (80)). Na Equação (18), necessita-se de uma expressão matemática para a espessura da camada limite de concentração, $\delta(x)$. Contudo, 
não encontramos na literatura uma formulação para “ $\delta(x)$ ” que atenda especificamente o contexto deste trabalho.

Para que a Equação (18) fique completamente determinada, construiremos uma expressão matemática para a espessura da camada limite de concentração, $\delta$. Assim, consideremos um escoamento em regime laminar e estacionário de um fluido newtoniano e incompressível. Vamos supor que a camada limite de concentração seja homogênea, e sem influência da concentração polarizada próxima a superfície permeável, ou seja, existe uma camada fina de espessura $\delta$ (análoga à utilizada na teoria clássica do filme). Sobre as partículas confinadas nesta fina camada, cada uma delas sofre a ação das seguintes forças envolvidas: difusão, interação, Lift-Force, forças de arrasto e de fricção (se a partícula estiver na superfície da membrana) (RIPPERGER e ALTMAN, 2002). Algumas características do escoamento central, fluxo transmembrana e fluido que estão associadas à espessura da camada limite de concentração são a densidade, a viscosidade, a difusividade, a velocidade média de entrada, a velocidade na parede e a pressão transmembrana.

A espessura da camada limite de concentração varia com a posição axial, $\tilde{x}$, assim podemos escrever temos: $\delta=\delta(\tilde{\mathrm{x}})$ (RIPPERGER e ALTMAN, 2002; DAMAK et al., 2004; SONG e ELIMELECH, 1995). Com a finalidade de termos um específico controle sobre $\delta=\delta(\tilde{\mathrm{x}})$, através de uma equação, vamos supor que exista uma reta assintótica para a espessura da camada limite de concentração (camada fina) e que esta reta assintótica dependa de uma taxa, denotada por $1,0<1<1$, a qual será chamada de taxa assintótica, assim:

$$
\lim _{\mathrm{x} \rightarrow \infty} \delta(\tilde{\mathrm{x}})=\mathrm{\imath} \mathrm{R}_{0}, \quad 0<1<1
$$

com $\mathrm{R}_{0}$ sendo o diâmetro do tubo. Isto significa que, para um tubo infinito (ideal), $\delta=\delta(\tilde{\mathrm{x}})$ 
não ultrapassará o valor $\mathrm{R}_{0}$. Assim, a espessura da camada fina depende da taxa assintótica, $\imath, \operatorname{logo}, \delta=\delta(\imath, \tilde{\mathrm{x}})$, para $0<\imath<1$.

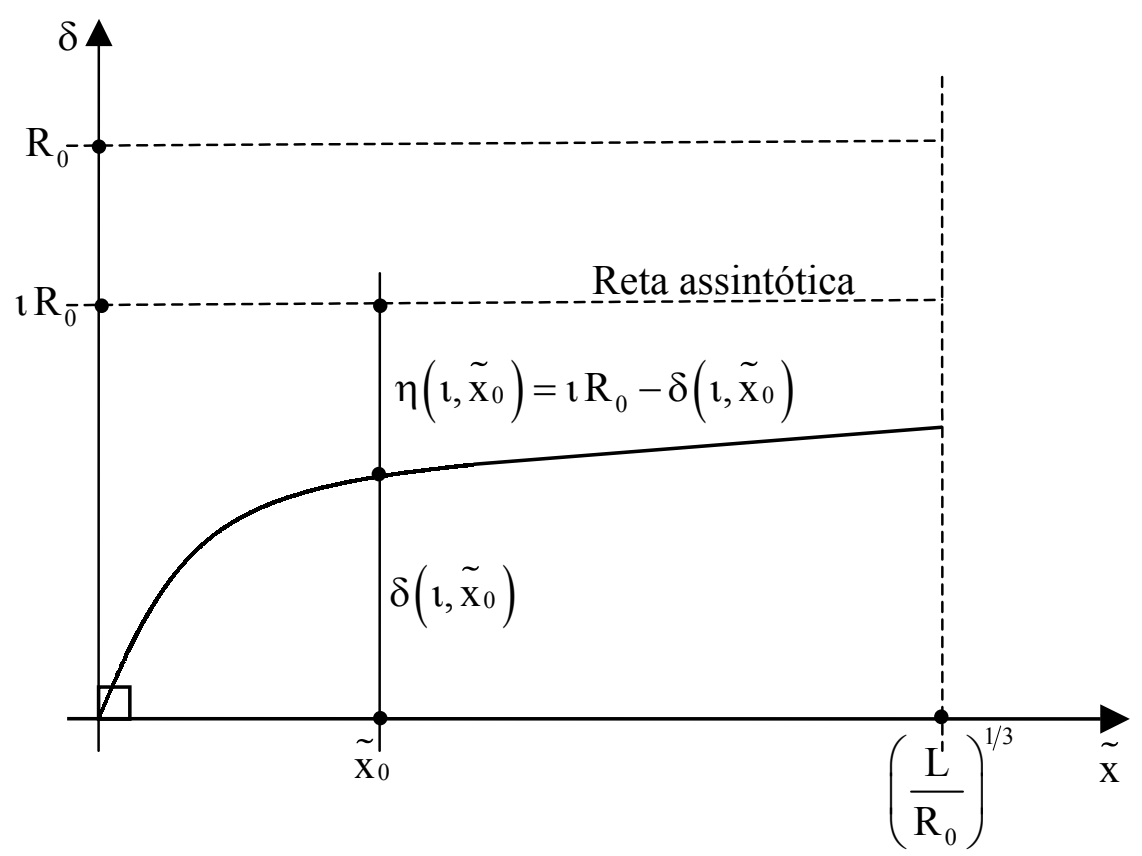

Figura 9. Espessura da camada limite de concentração variando com posição axial " $\tilde{x}$ ” e a representação da reta assintótica.

Sem perder a generalidade, tomemos o ponto no eixo das abscissas, cuja distância da origem equivale a $\left(\frac{\mathrm{L}}{\mathrm{R}_{0}}\right)^{1 / 3}$, com $\mathrm{L}>0$ e $\mathrm{R}_{0}>0$. Assim, para qualquer valor " $\tilde{\mathrm{x}}_{0}$ " pertencente ao intervalo $0 \leq \tilde{\mathrm{x}} \leq\left(\frac{\mathrm{L}}{\mathrm{R}_{0}}\right)^{1 / 3}$, existirá uma diferença entre a espessura da camada limite de concentração e a reta assintótica, dada por (Figura 9):

$$
\eta\left(\mathrm{l}, \tilde{\mathrm{x}}_{0}\right)=\mathrm{\imath} \mathrm{R}_{0}-\delta\left(\mathrm{l}, \tilde{\mathrm{x}}_{0}\right), \quad 0<\mathrm{\imath}<1
$$

Como vimos acima, $\imath \mathrm{R}_{0}>\delta(\mathrm{\imath}, \tilde{\mathrm{x}}), \quad 0 \leq \tilde{\mathrm{x}} \leq\left(\frac{\mathrm{L}}{\mathrm{R}_{0}}\right)^{1 / 3}$, e, a partir da Equação (91), podemos escrever uma expressão para a espessura da camada limite de concentração, vinculada à taxa assintótica, como segue: 


$$
\delta(1, \tilde{\mathrm{x}})=\mathrm{\imath} \mathrm{R}_{0}-\eta(\mathrm{l}, \tilde{\mathrm{x}})
$$

Analisando empiricamente a Equação (92), concluímos que $\eta(\mathfrak{\imath}, \tilde{\mathrm{x}})$ é proporcional à curvatura de uma curva parametrizada espiral logarítmica, a qual pode ser escrita na forma:

$$
\eta(\mathrm{l}, \tilde{\mathrm{x}})=\frac{1}{1+\mathrm{a} \tilde{\mathrm{x}}} \mathrm{l} \mathrm{R}_{0}, \quad 0<\imath<1 \quad \text { e } \quad 0 \leq \tilde{\mathrm{x}} \leq\left(\frac{\mathrm{L}}{\mathrm{R}_{0}}\right)^{1 / 3}
$$

com "a" sendo um escalar positivo. $\mathrm{Na}$ Equação (93), faremos uma conveniente transformação de coordenadas, como segue:

$$
\tilde{\mathrm{x}}=\left(\frac{\mathrm{x}}{\mathrm{R}_{0}}\right)^{1 / 3}, \quad 0 \leq \mathrm{x} \leq \mathrm{L}
$$

A Equação (92) será escrita na forma:

$$
\delta(\mathrm{l}, \mathrm{x})=\mathrm{l} \mathrm{R}_{0}-\frac{1}{1+\mathrm{a}\left(\frac{\mathrm{x}}{\mathrm{R}_{0}}\right)^{1 / 3}} \mathrm{l} \mathrm{R}_{0}, \quad 0<\mathrm{l}<1 \quad \text { e } \quad 0 \leq \mathrm{x} \leq \mathrm{L}
$$

Por análise empírica, verifica-se que o escalar "a" tem a seguinte forma:

$$
\mathrm{a}=\left(\operatorname{Re} \operatorname{Sc} \operatorname{Re}_{\mathrm{w}}\right)^{-1 / 3}=\left(\operatorname{Pe~Re}_{\mathrm{w}}\right)^{-1 / 3}
$$

A Equação (95) pode ser escrita na forma:

$$
\delta(\mathrm{l}, \mathrm{x})=\left(1-\frac{1}{1+\left(\frac{1}{{\operatorname{ReSc} \mathrm{Re}_{\mathrm{w}}}_{\mathrm{R}}} \frac{\mathrm{x}}{\mathrm{R}_{0}}\right)^{1 / 3}}\right) \mathrm{tR}_{0}
$$

com $0<1<1$ e $0 \leq \mathrm{x} \leq \mathrm{L}$.

Determinamos assim uma expressão matemática, Equação (97), para a espessura da camada limite de concentração local, associada a uma taxa assintótica.

A Equação (97) considera o comportamento da velocidade do fluido na parede através 
do número de Reynolds na parede, $\mathrm{Re}_{\mathrm{w}}$, assim os resultados obtidos desta equação envolvem problemas, cuja velocidade na parede é uniforme e dada a priori, conforme discutido na Seção (2.1.7). Esta equação não considera a resistência concentração polarizada e a resistência da membrana, contudo estas podem ser incorporadas no problema, através de "Re $\mathrm{w}_{\mathrm{w}}$, utilizando o modelo de resistência em série (Seção (2.1.9)).

\subsubsection{METOdOlogia PARA DETERMinaÇÃo da TAXA ASSINTÓtiCa}

Na seção anterior formulamos uma expressão matemática para a espessura da camada limite de concentração, $\delta$, contudo a Equação (18) ainda não está completamente determinada, e isto é devido à ocorrência da taxa assintótica.

Nesta seção desenvolveremos uma metodologia para determinar a taxa assintótica (Figura 10) e, a partir desta, avaliar a espessura da camada limite de concentração, $\delta$, e a concentração de polarização, $\mathrm{c}_{\mathrm{w}}$. Os passos da metodologia utilizada estão detalhados na seqüência:

(1) Início do processo;

(2) Entrar com os parâmetros: velocidade axial média de entrada, $\mathrm{u}_{0}$, coeficiente de difusão, $\mathrm{D}$, velocidade (uniforme) na parede, $\mathrm{V}_{\mathrm{w}}$, taxa assintótica inicial, 1 , erro percentual máximo, $\varepsilon=0.01$, outros parâmetros para o cálculo dos adimensionais $\mathrm{Re}, \mathrm{Sc}, \mathrm{Re}_{\mathrm{w}}$, espessura da camada limite de concentração;

(3) Entrar com os perfis de velocidade dados na Seção (2.1.7);

(4) Estabelecer a equação convectiva-difusiva de interesse (uma das Equações, de (19) até (21));

(5) Resolver a equação do passo (4) utilizando a GITT;

(6) Avaliar o número de Sherwood local, Sh (Equação (43)), o fluxo transmembrana local, 
$\mathrm{J}(\mathrm{X})$ (Equação (47)) e o fluxo médio, $\mathrm{J}_{\mathrm{m}}=\frac{1}{\mathrm{~L}} \int_{0}^{\mathrm{L}} \mathrm{J}(\mathrm{X}) \mathrm{dX}$;

(7) Verificar se:

(7.1) Se $\mathrm{J}_{\mathrm{m}}<(1-\varepsilon) \mathrm{v}_{\mathrm{w}}$, então $\mathrm{\imath}=\imath+\Delta \quad(\Delta$ : incremento $)$ ou;

(7.2) Se $J_{m}>(1+\varepsilon) \mathrm{V}_{\mathrm{w}}$, então $\imath=\imath-\Delta \quad(\Delta$ : decremento $)$;

(7.3) Ocorrendo qualquer um dos casos (7.1) ou (7.2), o processo deve ser feito novamente a partir do passo 2;

(8) Não ocorrendo qualquer um dos casos (7.1) ou (7.2), então determinamos a taxa assintótica, 1 ;

(9) Fim do processo.

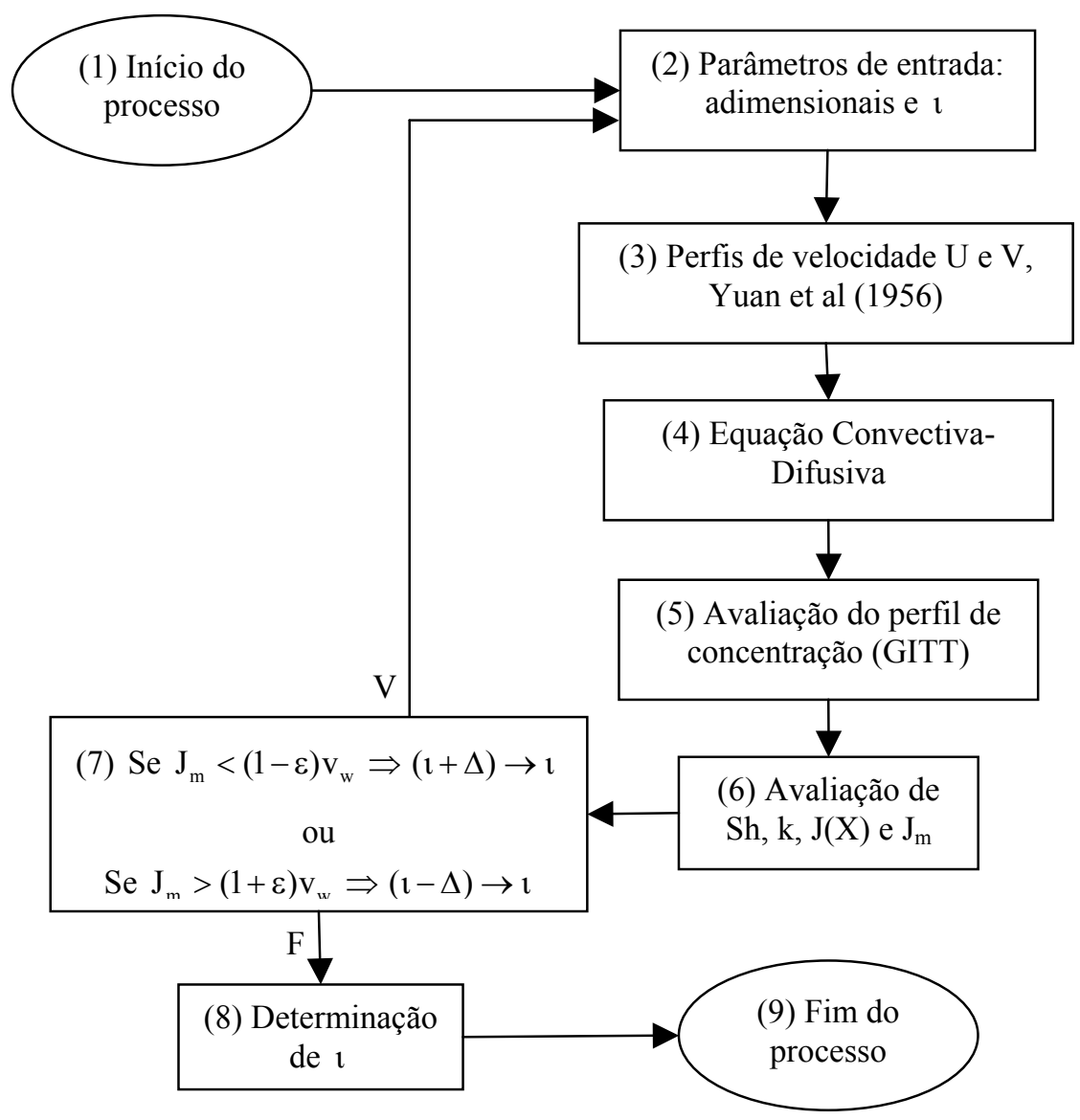

Figura 10. Representação esquemática da metodologia da modelagem que envolve 9 passos. No passo (2), a espessura da camada limite de concentração polarizada, $\delta(\iota, X)$, e a concentração na parede, $\mathrm{C}_{\mathrm{w}}(\mathrm{X})$, são avaliadas para uma taxa assintótica, ı, inicial, as quais serão ajustadas no final do processo segundo o critério do passo (7). 
No passo (2), considera-se uma estimativa para a taxa assintótica inicial, que satisfaça o critério estabelecido na Seção (3.3.3), ou seja, a taxa assintótica deve ser menor que a taxa assintótica máxima, e para esta taxa assintótica máxima, desenvolveremos uma expressão matemática na próxima seção. A partir dos parâmetros de entrada, juntamente com a taxa assintótica inicial, podemos avaliar a espessura da camada limite de concentração, e a partir daí, avaliarmos também a concentração polarizada. Este último resultado estabelece a condição de contorno na parede, $\mathrm{R}=1$.

No passo (5) a GITT resolve uma respectiva equação convectiva-difusiva. Para os parâmetros estabelecidos no passo (1), o perfil de concentração obtido via GITT é uma solução quase-exata, mas ainda não podemos afirmar que este resultado analítico-numérico é o mais próximo do fenômeno envolvido neste processo de filtração tangencial, pois trabalhamos com o modelo (gel polarizado) aproximado e uma metodologia que tem como critério a teoria clássica do filme.

Seguindo a metodologia desta seção, no passo (6) avaliamos o fluxo médio, $\mathrm{J}_{\mathrm{m}}$, o qual deve pertencer ao intervalo fechado $I=\left[(1-\varepsilon) \mathrm{v}_{\mathrm{w}},(1+\varepsilon) \mathrm{v}_{\mathrm{w}}\right]$. Esta comparação pode ser feita, pois " $\mathrm{J}_{\mathrm{m}}$ " é calculado a partir do modelo gel polarizado (Seção (2.1.9)). Este modelo foi desenvolvido a partir da teoria clássica do filme (Seção (2.1.4)) que tem como condição de contorno: $\mathrm{V}_{\mathrm{w}}=\mathrm{J}$ (Equação (26)). Devido à inconsistência existente no adimensional de Sherwood, Equação (43), na entrada do tubo, $X=0$, avaliamos $J(X)$ na posição axial $X=$ $0,10 \xi$

Um critério de erro entre " $\mathrm{J}_{\mathrm{m}}$ " e " $\mathrm{v}_{\mathrm{w}}$ " foi estabelecido no passo (7) pelo parâmetro $\varepsilon$, que delimita o quanto se espera que estas grandezas estejam próximas. A atribuição do erro percentual máximo, $\varepsilon$, depende do quanto são representativas as condições dadas na modelagem, e neste trabalho utilizamos $\varepsilon=0,01$. A Figura 11 mostra o intervalo determinado 
por " $\mathrm{V}_{\mathrm{w}}$ " e pelo erro percentual, $\varepsilon$. O processo iterativo encerra-se quando $\mathrm{J}_{\mathrm{m}} \in \mathrm{I}$; caso contrário $\mathrm{J}_{\mathrm{m}}>(1+\varepsilon) \mathrm{v}_{\mathrm{w}}$ ou $\mathrm{J}_{\mathrm{m}}<(1+\varepsilon) \mathrm{v}_{\mathrm{w}}$. Ocorrendo o primeiro caso, devemos retornar no passo (2), mas atribuindo a taxa assintótica um decremento $\Delta$. Analogamente, para o segundo caso, retornamos para o passo (2) atribuindo um incremento $\Delta$ a taxa assintótica. Observamos que $\imath+\Delta$ não poderá ser maior que a taxa assintótica máxima, a qual será formulada na Seção (3.3.3).

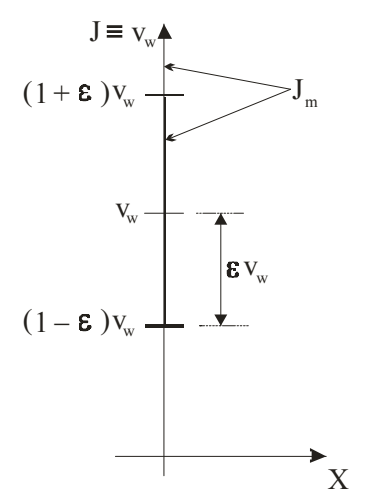

Figura 11. Esquema para o intervalo $\mathrm{I}=\left[(1-\varepsilon) \mathrm{V}_{\mathrm{w}},(1+\varepsilon) \mathrm{V}_{\mathrm{w}}\right]$ relacionando " $\mathrm{J}_{\mathrm{m}}$ " $\mathrm{e}$ " $\mathrm{V}_{\mathrm{w}}$ ".

\subsubsection{TaXa Assintótica MáXima}

Na Equação (50) temos a condição de contorno não homogênea na parede do tubo, $\mathrm{C}_{\mathrm{w}}(\mathrm{x})=\mathrm{c}_{\mathrm{w}}(\mathrm{x}) / \mathrm{c}_{0}$, a qual é definida pela Equação (18). Estas equações dependem, entre outros parâmetros, da espessura da camada limite de concentração local, $\delta(x)$. Contudo, a expressão que desenvolvemos para a espessura da camada limite de concentração, $\delta(1, x)$, na Seção (3.3.1) (Equação (97)), está associada à taxa assintótica, 1 .

Na metodologia da Seção (3.3.2) temos um processo iterativo para determinação da taxa assintótica. Dependendo dos parâmetros iniciais, a atribuição de uma taxa assintótica inicial qualquer pode levar à incoerência na concentração na parede, ou seja, pode ocorrer valor de " $\mathrm{C}_{\mathrm{w}}(\mathrm{x})$ " infinito, positivo e negativo, ou pode ocorrer ponto de inflexão no gráfico de 
" $\mathrm{C}_{\mathrm{w}}(\mathrm{x})$ " e, isto ocorrendo, gera transtornos durante o processamento computacional. Desta forma, determinaremos nesta seção uma taxa assintótica máxima que garante a não ocorrência de inconsistências em " $\mathrm{C}_{\mathrm{w}}(\mathrm{x})$ ", para toda taxa assintótica inicial menor que a referida taxa máxima.

Nos trabalhos de Geraldes et al. (2001) e Damak et al. (2004 e 2004A) é mostrado que a espessura da camada limite de concentração é sensível ao número de Reynolds, Re, número de Reynolds de permeação, $\mathrm{Re}_{\mathrm{w}}$ e número de Schmidt, Sc. A função, $\mathrm{c}_{\mathrm{w}}$, definida pela Equação (18) deve ser assumida sendo monótona crescente com relação ao eixo de simetria, e seu gráfico tem a concavidade voltada para baixo, para qualquer posição axial (DAMAK et al., 2004; PARIS et al., 2002). Além disso, a concentração polarizada é um fenômeno de intensidade maior (ou igual) à concentração de entrada, $\mathrm{c}_{0}$, para toda posição axial. Assim, temos os seguintes critérios:

$$
\begin{array}{ll}
\frac{\partial^{2} c_{w}(x)}{\partial x^{2}}<0 & 0<x<L \quad \text { e } \\
c_{w}(x) \geq c_{0} & 0 \leq x \leq L
\end{array}
$$

Fixaremos todos os parâmetros $\mathrm{Re}, \mathrm{Re}_{\mathrm{w}}, \mathrm{Sc}$ e $\mathrm{R}_{0}$ e avaliaremos a taxa assintótica na saída do tubo, $\mathrm{X}=\xi=\mathrm{L} / \mathrm{R}_{0}$, onde a camada limite de concentração é a maior possível, e assim estabelecermos sua espessura, $\delta$, também máxima. O critério, dado pela Equação (98), pode ser escrito, na forma adimensional, como:

$$
\left.\frac{\partial^{2} C_{w}}{\partial X^{2}}\right|_{x=\xi}<0
$$

Com objetivo de calcularmos a taxa assintótica, a partir da Equação (100), vamos supor que: 


$$
\left.\frac{\partial^{2} C_{w}}{\partial X^{2}}\right|_{x=\xi}=0
$$

Na Equação (101) substituímos as Equações (18) e (97), e assim efetuamos o cálculo diferencial de segunda ordem utilizando a plataforma de manipulação simbólica MATHEMATICA 5.2 (2005), pela qual obtemos a seguinte equação:

$$
\frac{-2 a R_{0} v_{w} D\left(D+2 a\left(D-R_{0} v_{w} l\right) \xi^{1 / 3}\right) \imath}{9\left(D+a\left(D-R_{0} v_{w} l\right) \xi^{1 / 3}\right)^{3} \xi^{5 / 3}}=0
$$

com "a" sendo dado pela Equação (96). A Equação (102) é resolvida, com relação à taxa assintótica, 1, utilizando o comando SOLVE da plataforma de manipulação simbólica Mathematica 5.2 (2005). Desta forma, determinamos uma taxa assintótica na saída do tubo, $\mathrm{X}=\xi=\mathrm{L} / \mathrm{R}_{0}$, o qual é sugerido chamá-la de taxa assintótica máxima, denotada por: $\mathrm{l}_{\max }$, e obtida como:

$$
\mathrm{l}_{\max }=\left(1+\frac{1}{2\left(\frac{\xi}{\operatorname{ReScRe}_{\mathrm{w}}}\right)^{1 / 3}}\right) \frac{1}{\mathrm{ScRe}_{\mathrm{w}}}
$$

Assim podemos estabelecer um intervalo aberto $\left(0, l_{\max }\right)$ onde os critérios dados pelas Equações (98) e (99) são satisfeitos simultaneamente. Portanto, a condição de contorno na parede da modelagem deste trabalho (Equação (24) e Equação (50)), ou seja, a concentração na parede, $\mathrm{c}_{\mathrm{w}}$, para um perfil de concentração máximo estabelecido pela taxa assintótica máxima, fica coerente com os fundamentos teóricos. 



\section{Resultados e Discussões}

\subsection{INTRODUÇÃO}

Para simularmos o problema físico proposto neste trabalho, estabeleceremos intervalos para os parâmetros que descrevem as características da geometria, do fluido e do escoamento. Para tal, avaliamos os parâmetros dos trabalhos de Paris et al. (2002) e Yeh et al. (2004), os quais apresentam resultados numéricos e experimentais do fluxo transmembrana, e do trabalho de Damak et al. (2004), que traz resultados somente numéricos do adimensional de Sherwood. A velocidade de permeação uniforme, $\mathrm{v}_{\mathrm{w}}$, também pode ser determinada através da lei de Poiseuille (Equação (3)) ou modelo de resistência em série (Equação (42)), utilizando os parâmetros que descrevem os fenômenos da Microfiltração e Ultrafiltração.

Consideramos os seguintes parâmetros:

- Geometria:

$\mathrm{L}=0,3 \mathrm{~m}$

$\mathrm{R}_{0}=0,003 \mathrm{~m} ;$ 
- Fluido:

$\rho=1,010^{3} \mathrm{~kg} \mathrm{~m}^{-3}$

$\mu=1,110^{-3} \mathrm{~kg} \mathrm{~m}^{-1} \mathrm{~s}^{-1}$

$4,410^{-10} \mathrm{~m}^{2} \mathrm{~s}^{-1} \leq \mathrm{D} \leq 1,3810^{-9} \mathrm{~m}^{2} \mathrm{~s}^{-1}$

- Escoamento:

$7,310^{-2} \mathrm{~ms}^{-1} \leq \mathrm{u}_{0} \leq 25,610^{-2} \mathrm{~ms}^{-1}$

$1,810^{-6} \mathrm{~ms}^{-1} \leq \mathrm{v}_{\mathrm{w}} \leq 6,210^{-6} \mathrm{~ms}^{-1}$

A partir destes parâmetros, estabelecemos os intervalos para os seguintes adimensionais: $800 \leq \mathrm{Sc} \leq 2500,200 \leq \mathrm{Re} \leq 700$ e $0,510^{-2} \leq \mathrm{Re}_{\mathrm{w}} \leq 1,410^{-2}$.

Na equação convectiva-difusiva elíptica (modelo elíptico), equação convectiva-difusiva parabólica (modelo parabólico), respectivamente conforme as Seções (3.1) e (3.2), existe a ocorrência do número de Péclet, $\mathrm{Pe}=\mathrm{u}_{0} \mathrm{R}_{0} / \mathrm{D}=\mathrm{Re} \mathrm{Sc}$, para o qual fica estabelecido o intervalo $160000 \leq \mathrm{Pe} \leq 1750000$. O número de Reynolds na parede permeável, $\mathrm{Re}_{\mathrm{w}}$, é utilizado na determinação dos perfis de velocidade U e V. O número de Schmidt, Sc, é usado no cálculo da taxa assintótica, $\mathrm{l}$, através do parametro $\mathrm{a}=\left(\operatorname{Re} \operatorname{Sc}_{\mathrm{W}}\right)^{-1 / 3}$.

De forma análoga à realizada acima, podemos estabelecer outros intervalos para os parâmetros " $\mathrm{v}_{\mathrm{w}}$ ", "u $\mathrm{u}_{0}$ " e "D", ampliando assim a faixa de interesse da filtração tangencial.

Analisaremos o comportamento do perfil de concentração e do número de Sherwood para cada um dos modelos mencionados acima e também os compararemos com outros resultados da literatura. $\mathrm{Na}$ última seção deste capítulo, analisaremos também o 
comportamento da espessura da camada limite de concentração e da concentração polarizada.

A partir dos conceitos existentes na Seção (2.1), Mecanismo de transferência de massa na filtração tangencial, observações podem ser feitas sobre os efeitos físicos do perfil de concentração, C, do número de Sherwood, Sh, da espessura da camada limite de concentração, $\delta$, e da concentração polarizada, $\mathrm{C}_{\mathrm{w}}$, quando variamos os adimensionais: Reynolds, Re; Reynolds de permeação, $\mathrm{Re}_{\mathrm{w}}$, e Schmidt, Sc.

Aumentando "Re", o transporte convectivo de soluto através da camada limite de concentração se intensificará, e o transporte difusivo será reduzido, causando uma diminuição na "C", uma diminuição na " $\mathrm{C}_{\mathrm{w}}$ " e também na " $\delta$ ". O número de Sherwood, Sh, é inversamente proporcional a " $\mathrm{C}_{\mathrm{w}}$ " (Equação (43)), e com a diminuição de "C $\mathrm{C}_{\mathrm{w}}$ ", "Sh" aumentará.

A relação entre "Sc" e a difusividade, D, é inversamente proporcional, ou seja, o aumento de "Sc" pode estar associado à diminuição do coeficiente de difusão "D". Com a diminuição de "D”, tem-se a diminuição da contribuição difusiva de transferência de massa junto à parede permeável, conseqüentemente ocorrerá à formação de espessuras mais delgadas da camada limite de concentração, $\delta$, e também o aumento de "C $\mathrm{C}_{\mathrm{w}}$ " e de "C". "Sh" (=k $\mathrm{R}_{0} / \mathrm{D}$ ) é inversamente proporcional a " $\mathrm{D}$ ”. Com a diminuição de " $\mathrm{D}$ ” ocorrerá o aumento de "Sc" e daí o aumento de "Sh".

$\mathrm{O}$ número de Reynolds de permeação, $\mathrm{Re}_{\mathrm{w}}$, é diretamente proporcional à velocidade na parede, $\mathrm{v}_{\mathrm{w}}$. $\mathrm{O}$ aumento de " $\mathrm{v}_{\mathrm{w}}$ " pode ser causado pelo aumento da contribuição convectiva de transporte de massa e pela diminuição do transporte difusivo nas adjacências da superfície permeável, produzindo a diminuição na " $\delta$ ”, o aumento de " $\mathrm{C}_{\mathrm{w}}$ ” e o aumento de "C $\mathrm{C}$ ". Através do modelo do gel, podemos concluir que "Sh" é diretamente proporcional ao produto de 
"Re $e_{\mathrm{w}}$ " e "Sc" $\left(\operatorname{Re}_{\mathrm{w}} \mathrm{Sc}=\mathrm{Sh} \ln \left(\mathrm{C}_{\mathrm{w} 0}\right)\right)$, assim com o aumento "Re $\mathrm{e}_{\mathrm{w}}$ " ocorrerá o aumento de "Sh".

A Tabela 2 mostra os efeitos físicos do perfil de concentração, C, do número de Sherwood, Sh, da espessura da camada limite de concentração, $\delta$, e da concentração polarizada, $\mathrm{C}_{\mathrm{w}}$, quando variamos os adimensionais: Reynolds, Re; Reynolds de permeação,

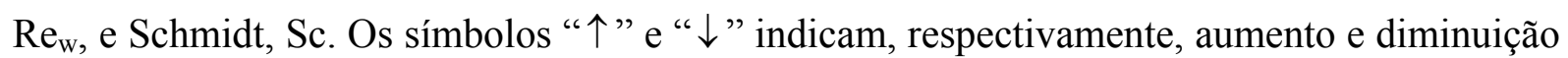
do adimensional representado à esquerda destes símbolos.

Tabela 2. Resumo do comportamento dos efeitos físicos envolvidos nos processos de filtração tangencial.

\begin{tabular}{|l|l|l|l|l|l|l|l|}
\cline { 2 - 8 } \multicolumn{1}{|c|}{ Adimensionais } & \multicolumn{4}{c|}{ Perfis } \\
\hline 1 & $\mathrm{Re}-\uparrow$ & $\mathrm{Sc}-$ fixo & $\mathrm{Re}_{\mathrm{w}}-$ fixo & $\mathrm{C}-\downarrow$ & $\mathrm{C}_{\mathrm{w}}-\downarrow$ & $\delta-\downarrow$ & $\mathrm{Sh}-\uparrow$ \\
\hline 2 & $\mathrm{Re}-$ fixo & $\mathrm{Sc}-\uparrow$ & $\mathrm{Re}_{\mathrm{w}}-$ fixo & $\mathrm{C}-\uparrow$ & $\mathrm{C}_{\mathrm{w}}-\uparrow$ & $\delta-\downarrow$ & $\mathrm{Sh}-\uparrow$ \\
\hline 3 & $\mathrm{Re}-$ fixo & $\mathrm{Sc}-$ fixo & $\mathrm{Re}_{\mathrm{w}}-\uparrow$ & $\mathrm{C}-\uparrow$ & $\mathrm{C}_{\mathrm{w}}-\uparrow$ & $\delta-\downarrow$ & $\mathrm{Sh}-\uparrow$ \\
\hline
\end{tabular}

\subsection{Resultados E Discussões}

Os autovalores e as integrais de normalização, Equações (63) e (66), respectivamente, para cada um dos modelos: equação convectiva-difusiva elíptica e equação convectivadifusiva parabólica foram determinados analiticamente usando a plataforma de manipulação simbólica MATHEMATICA 5.2 (2005) (ver o Apêndice 6, programa: “AutoVal_IntNorm”), considerando $\mathrm{R}_{\min }=10^{-23}$.

Os coeficientes integrais para cada modelo: equação convectiva-difusiva elíptica (Equação (72) até (78)), equação convectiva-difusiva parabólica, (Equação (84) até (88)), foram calculados numericamente, com uma tolerância de erro relativo prescrita de $10^{-7}$, usando a sub-rotina DQDGAS da biblioteca matemática do IMSL LIBRARY (1991) (ver o 
Apêndice 6, programas: “CoefIntegraEliptico" e "CoefIntegraParabolico", respectivamente).

A solução do sistema diferencial ordinário com condições de contorno em dois pontos (relativo à equação convectiva-difusiva elíptica), dado pela Equação (69), foi calculada pela sub-rotina DBVPFD do IMSL LIBRARY (1991), que utiliza uma ordem variável, método de diferença finita com tamanho dos passos variável com pós-correções. Esta sub-rotina oferece um controle automático de erro relativo local, prescrito pelo usuário, cuja tolerância usada neste trabalho foi de $10^{-4}$ (ver o Apêndice 6, programa: "ProgPrincEliptico").

A solução do sistema diferencial ordinário, relativos à equação convectiva-difusiva parabólica, dada pela Equação (83), foi calculada usando a sub-rotina DIVPAG do IMSL LIBRARY (1991), que utiliza um algoritmo com o método de Gear por diferenças finitas e precisão prescrita pelo usuário. No presente estudo foi utilizada uma tolerância de erro de $10^{-10}$ (ver o Apêndice 6, programa: "ProgPrincParabolico").

Os resultados obtidos desta modelagem do comportamento dos perfis de concentração e do adimensional de Sherwood serão analisados e comparados com outros resultados da literatura. Além disso, a partir da convergência global do perfil de concentração, para cada modelagem, será avaliado o número de termos "N" para truncamento da equação diferencial ordinária transformada.

\subsubsection{CONVERGÊNCIA GLOBAL}

Na Tabela 3 e na Tabela 4 temos os resultados do perfil de concentração para análise da convergência global, onde estabelecemos as ordens de truncamentos, bem como os respectivos tempos de processamento, para os dois tipos de modelagem: equação convectivadifusiva parabólica e equação convectiva-difusiva elíptica. Nestes casos, fixamos os adimensionais $\mathrm{Sc}=2500, \mathrm{Re}_{\mathrm{w}}=0,01, \mathrm{Re}=900$. Para outros valores dos adimensionais, $\mathrm{a}$ 
convergência dos resultados da concentração ocorrerá para a mesma ordem de truncamento.

A Tabela 3 apresenta resultados para a concentração, relacionados à equação convectiva-difusiva parabólica, na posição axial $\mathrm{R}=\mathrm{r} / \mathrm{R}_{0}=0,98$ em função da coordenada adimensional axial $\mathrm{X}\left(=\mathrm{x} / \mathrm{R}_{0}\right)$. Os valores da ordem de truncamento, $\mathrm{N}$, na expansão do perfil de concentração foram considerados sendo $10,15,25,35,45,46$ e 47 . Os resultados gerados a partir da formulação GITT reproduzem as soluções completamente convergidas e estabelecidas, que nesta modelagem exigiu-se um erro relativo global de $10^{-3}$. Observa-se na tabela que o comportamento da convergência da concentração fica estabelecido para "N" maior que 46.

Tabela 3. (Equação Convectiva-Difusiva Parabólica) Análise da convergência da concentração C, na posição radial $\mathrm{R}=0,98$ em função da coordenada axial $\mathrm{x} / \mathrm{R}_{0}$, com limite de tolerância igual a $10^{-3}, \mathrm{Sc}=2500, \mathrm{Re}_{\mathrm{w}}=0,01$, $\mathrm{Re}=900, \mathrm{R}_{0}=3,010^{-3} \mathrm{~m}$ e $\varepsilon=0,01$.

\begin{tabular}{cccccccc} 
& \multicolumn{7}{c}{$\mathrm{c} / \mathrm{c}_{0}$} \\
\cline { 2 - 7 } $\mathrm{N}$ & 10 & 15 & 25 & 35 & 45 & 46 & 47 \\
\hline $\mathrm{x} / \mathrm{R}_{0}$ & & & & & & \\
\hline 0 & 1,000 & 1,000 & 1,000 & 1,000 & 1,000 & 1,000 & 1,000 \\
10 & 1,209 & 1,123 & 1,061 & 1,045 & 1,043 & 1,042 & 1,042 \\
20 & 1,274 & 1,162 & 1,091 & 1,076 & 1,072 & 1,072 & 1,072 \\
30 & 1,323 & 1,191 & 1,117 & 1,100 & 1,094 & 1,093 & 1,093 \\
40 & 1,365 & 1,217 & 1,138 & 1,118 & 1,111 & 1,110 & 1,110 \\
50 & 1,401 & 1,239 & 1,157 & 1,134 & 1,126 & 1,125 & 1,125 \\
60 & 1,434 & 1,26 & 1,174 & 1,148 & 1,139 & 1,137 & 1,137 \\
70 & 1,465 & 1,28 & 1,189 & 1,160 & 1,151 & 1,149 & 1,149 \\
80 & 1,494 & 1,298 & 1,203 & 1,172 & 1,161 & 1,159 & 1,159 \\
90 & 1,522 & 1,315 & 1,215 & 1,182 & 1,171 & 1,168 & 1,168 \\
100 & 1,549 & 1,332 & 1,227 & 1,191 & 1,180 & 1,177 & 1,177 \\
\hline
\end{tabular}

A Tabela 4 apresenta resultados para a concentração, relacionados à equação convectiva-difusiva elíptica, na posição axial $\mathrm{R}=\mathrm{r} / \mathrm{R}_{0}=0,98$ em função da coordenada adimensional axial $\mathrm{X}\left(=\mathrm{x} / \mathrm{R}_{0}\right)$. Os valores de ordem de truncamento para, $\mathrm{N}$, na expansão do perfil de concentração foram considerados sendo $10,15,25,34,35$ e 37 . Os resultados gerados a partir da formulação GITT reproduzem a solução completamente convergida e estabelecida, que nesta modelagem exigiu-se um erro relativo global de $10^{-3}$. Observa-se na 
tabela que o comportamento da convergência da concentração fica estabelecido para "N" maior que 35.

Tabela 4. (Equação Convectiva-Difusiva Elíptica) Análise da convergência da concentração C, na posição radial $\mathrm{R}=0,98$ em função da coordenada axial $\mathrm{x} / \mathrm{R}_{0}$, com limite de tolerância igual a $10^{-3}, \mathrm{Sc}=2500, \mathrm{Re}_{\mathrm{w}}=0,01, \mathrm{Re}=900$,

$$
\mathrm{R}_{0}=3,010^{-3} \mathrm{~m} \text { e } \varepsilon=0,01 \text {. }
$$

\begin{tabular}{ccccccc}
\hline \multicolumn{7}{c}{$\mathrm{c} / \mathrm{c}_{0}$} \\
\hline $\mathrm{N}$ & 10 & 15 & 25 & 34 & 35 & 37 \\
\hline $\mathrm{x} / \mathrm{R}_{0}$ & & & & & & \\
\hline 0 & 1,000 & 1,000 & 1,000 & 1,000 & 1,000 & 1,000 \\
10 & 1,204 & 1,122 & 1,062 & 1,047 & 1,046 & 1,046 \\
20 & 1,267 & 1,160 & 1,092 & 1,080 & 1,079 & 1,079 \\
30 & 1,314 & 1,189 & 1,118 & 1,105 & 1,104 & 1,104 \\
40 & 1,354 & 1,214 & 1,140 & 1,126 & 1,124 & 1,124 \\
50 & 1,389 & 1,236 & 1,159 & 1,143 & 1,141 & 1,141 \\
60 & 1,421 & 1,257 & 1,176 & 1,158 & 1,156 & 1,156 \\
70 & 1,451 & 1,276 & 1,192 & 1,172 & 1,169 & 1,169 \\
80 & 1,479 & 1,294 & 1,206 & 1,184 & 1,182 & 1,182 \\
90 & 1,505 & 1,311 & 1,220 & 1,195 & 1,193 & 1,193 \\
100 & 1,531 & 1,327 & 1,232 & 1,206 & 1,203 & 1,203 \\
\hline
\end{tabular}

\subsubsection{ANÁlise dos Resultados}

Da Figura 12 até a Figura 23 são apresentados os resultados para o perfil de concentração e o adimensional de Sherwood em função da posição axial adimensional em tubo permeável, referentes à equação convectiva-difusiva elíptica e a equação convectivadifusiva parabólica, onde consideramos o erro percentual máximo sendo $\varepsilon=0,01$.

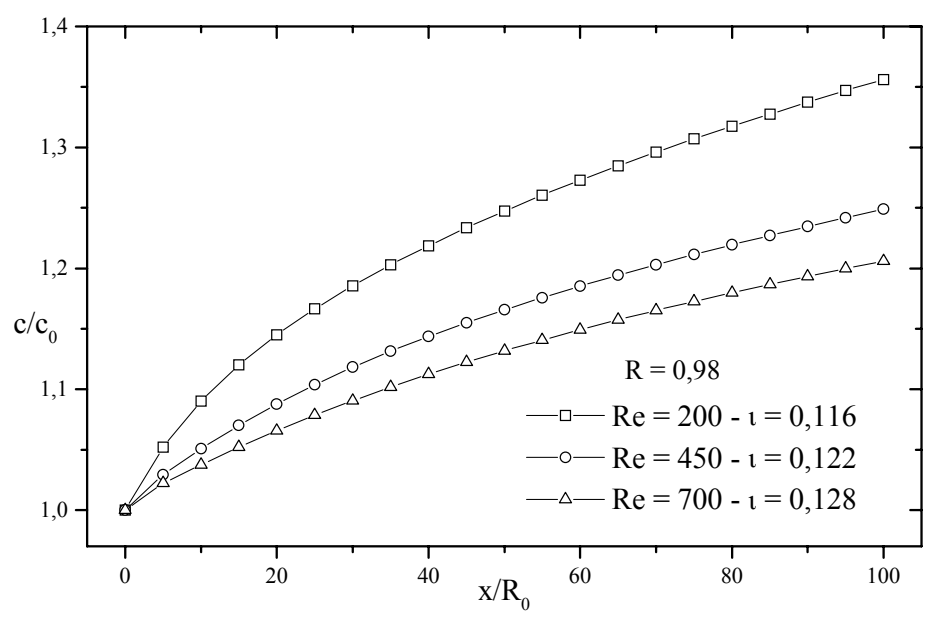

Figura 12. (Equação convectiva-difusiva elíptica) Efeito do número de Reynolds sobre o perfil de concentração em função da posição axial adimensional $\left(\mathrm{x} / \mathrm{R}_{0}\right)$ próximo à superfície permeável $(\mathrm{R}=0,98)$ para $\mathrm{Re}_{\mathrm{w}}=0,005$, $\mathrm{Sc}=2500, \mathrm{R}_{0}=3,010^{-3} \mathrm{~m}$ e N$=35$. 


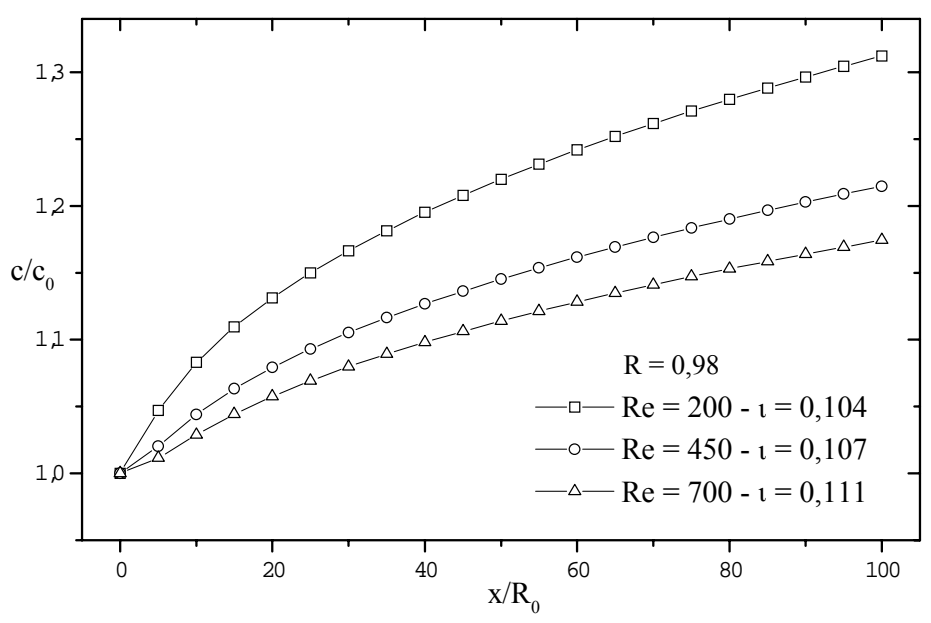

Figura 13. (Equação convectiva-difusiva parabólica) Efeito do número de Reynolds sobre o perfil de concentração em função da posição axial adimensional $\left(\mathrm{x} / \mathrm{R}_{0}\right)$ próximo à superfície permeável $(\mathrm{R}=0,98)$ para $\mathrm{Re}_{\mathrm{w}}=0,005, \mathrm{Sc}=2500, \mathrm{R}_{0}=3,010^{-3} \mathrm{~m}$ e $\mathrm{N}=46$.

Na Figura 12 e Figura 13 são apresentados resultados da concentração, C, próxima à superfície do tubo $(\mathrm{R}=0,98)$ em função da posição axial adimensional $\left(\mathrm{x} / \mathrm{R}_{0}\right)$ referentes à equação convectiva-difusiva elíptica e equação convectiva-difusiva parabólica. O comportamento destes perfis com relação às respectivas variações do adimensional "Re" está de acordo com a análise feita na $1^{\mathrm{a}}$ linha da Tabela 2.

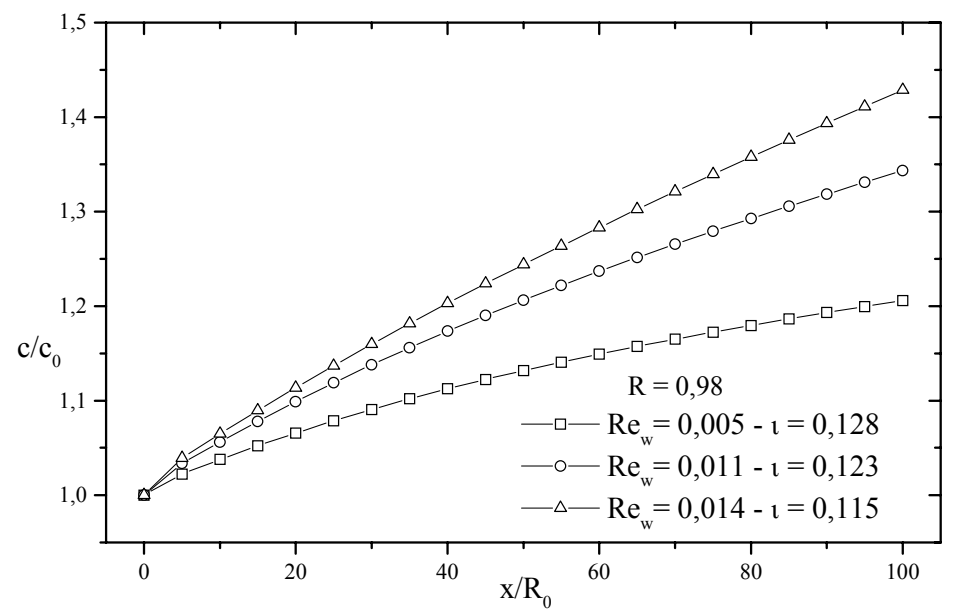

Figura 14. (Equação convectiva-difusiva elíptica) Efeito do número de Reynolds de permeação sobre o perfil de concentração em função da posição axial adimensional $\left(\mathrm{x} / \mathrm{R}_{0}\right)$ próximo à superfície permeável $(\mathrm{R}=0,98)$ para $\mathrm{N}=35, \mathrm{Re}=700, \mathrm{Sc}=2500$ e $\mathrm{R}_{0}=3,010^{-3} \mathrm{~m}$. 


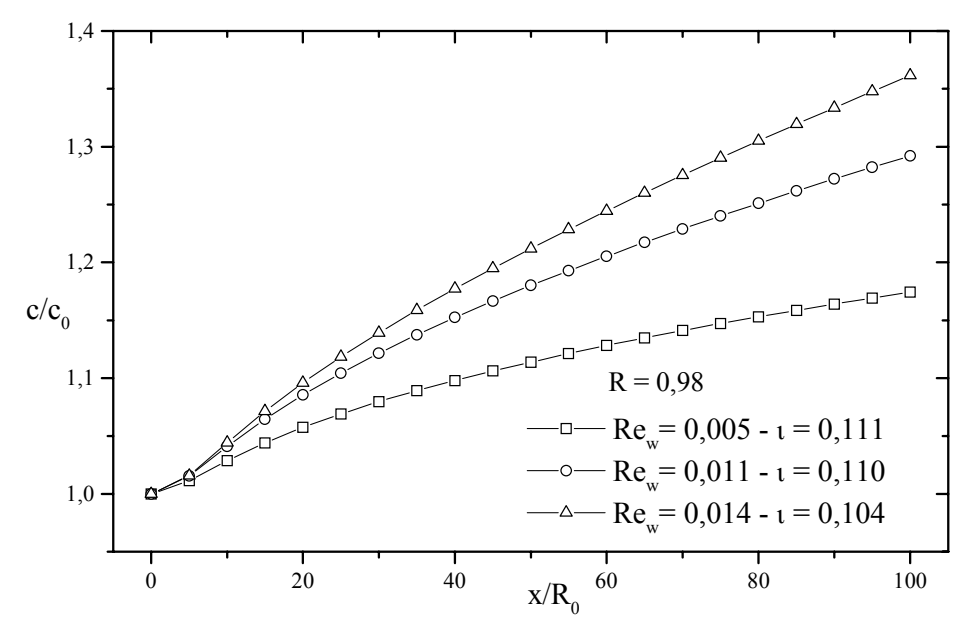

Figura 15. (Equação convectiva-difusiva parabólica) Efeito do número de Reynolds de permeação sobre o perfil de concentração em função da posição axial adimensional $\left(\mathrm{x} / \mathrm{R}_{0}\right)$ próximo à superfície permeável $(\mathrm{R}=0,98)$ para $\mathrm{N}=46, \mathrm{Re}=700, \mathrm{Sc}=2500$ e $\mathrm{R}_{0}=3,010^{-3}$.

Na Figura 14 e Figura 15 são apresentados resultados da concentração, C, próxima à superfície do tubo $(\mathrm{R}=0,98)$ em função da posição axial adimensional $\left(\mathrm{x} / \mathrm{R}_{0}\right)$ referentes à equação convectiva-difusiva elíptica e equação convectiva-difusiva parabólica. $\mathrm{O}$ comportamento destes perfis com relação às respectivas variações do adimensional " $\mathrm{Re}_{\mathrm{w}}$ " está de acordo com a análise feita na $3^{\text {a }}$ linha da Tabela 2.

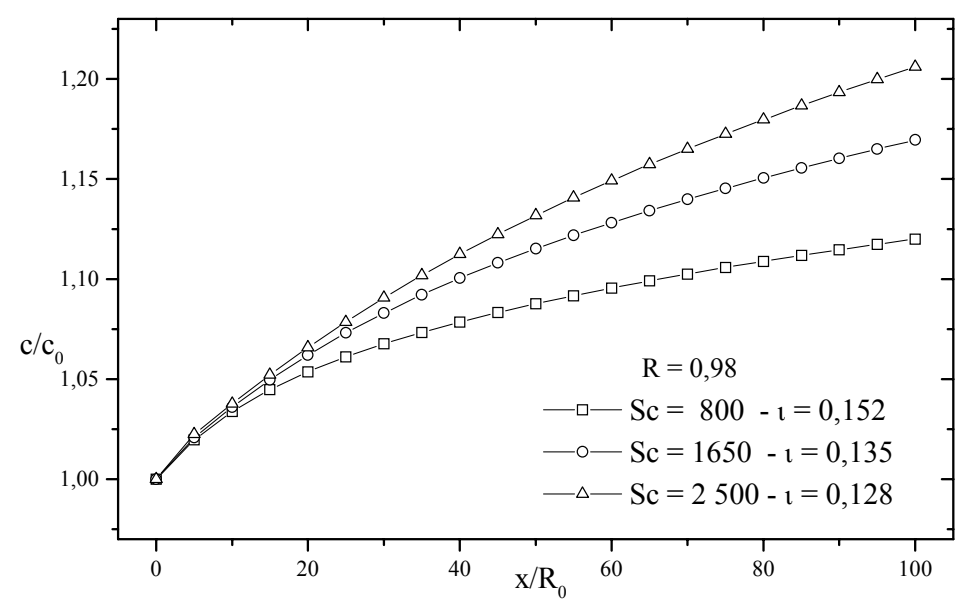

Figura 16. (Equação convectiva-difusiva elíptica) Efeito do número de Schmidt sobre o perfil de concentração em função da posição axial adimensional $\left(\mathrm{x} / \mathrm{R}_{0}\right)$ próximo à superfície permeável $(\mathrm{R}=0,98)$ para $\mathrm{N}=35, \mathrm{Re}=700$, $\mathrm{Re}_{\mathrm{w}}=0,005$ e $\mathrm{R}_{0}=3,010^{-3} \mathrm{~m}$. 


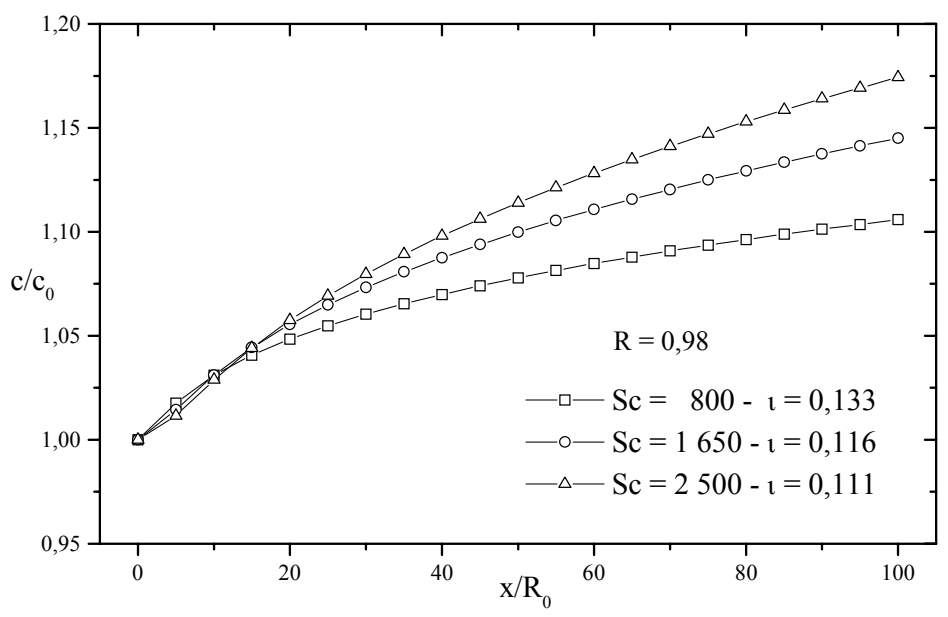

Figura 17. (Equação convectiva-difusiva parabólica) Efeito do número de Schmidt sobre o perfil de concentração em função da posição axial adimensional $\left(\mathrm{x} / \mathrm{R}_{0}\right)$ próximo à superfície permeável $(\mathrm{R}=0,98)$ para $\mathrm{Re}=700$, $\mathrm{Re}_{\mathrm{w}}=0,005, \mathrm{~N}=46$ e $\mathrm{R}_{0}=310^{-3} \mathrm{~m}$.

$\mathrm{Na}$ Figura 16 e Figura 17 são apresentados resultados da concentração, $\mathrm{C}$, próxima à superfície do tubo $(\mathrm{R}=0,98)$ em função da posição axial adimensional $\left(\mathrm{x} / \mathrm{R}_{0}\right)$ referentes à equação convectiva-difusiva elíptica e equação convectiva-difusiva parabólica. O comportamento destes perfis com relação às respectivas variações do adimensional "Sc" está de acordo com a análise feita na $2^{\mathrm{a}}$ linha da Tabela 2.

Tabela 5. Resumo das comparações da concentração, na saída do tubo, $\mathrm{L} / \mathrm{R}_{0}=100$, entre o modelo elíptico e parabólico. Indicação da diferença percentual.

\begin{tabular}{ccccc}
\cline { 2 - 5 } & \multicolumn{4}{c}{$\mathrm{C}$} \\
\cline { 2 - 5 } Modelo & $\mathrm{Re}=700$ & $\mathrm{Re}=200$ & $\mathrm{Re}=450$ & $\mathrm{Re}=700$ \\
& $\mathrm{Re}_{\mathrm{w}}=0,014$ & $\mathrm{Re}_{\mathrm{w}}=0,005$ & $\mathrm{Re}_{\mathrm{w}}=0,005$ & $\mathrm{Re}_{\mathrm{w}}=0,005$ \\
& $\mathrm{Sc}=2500$ & $\mathrm{Sc}=2500$ & $\mathrm{Sc}=2500$ & $\mathrm{Sc}=2500$ \\
\hline Elíptico & 1,43 & 1,36 & 1,25 & 1,21 \\
\hline Parabólico & 1,36 & 1,31 & 1,21 & 1,17 \\
\hline Dif. percentual & $5 \%$ & $3 \%$ & $3 \%$ & $3 \%$ \\
\hline
\end{tabular}

Tabela 6. Continuação do resumo das comparações da concentração, na saída do tubo, $\mathrm{L} / \mathrm{R}_{0}=100$, entre o modelo elíptico e parabólico. Indicação da diferença percentual.

\begin{tabular}{lccc}
\cline { 2 - 4 } Modelo & \multicolumn{3}{c}{$\mathrm{C}$} \\
\cline { 2 - 4 } & $\begin{array}{c}\mathrm{Re}=700 \\
\mathrm{Re}_{\mathrm{w}}=0,005 \\
\mathrm{Sc}=800\end{array}$ & $\begin{array}{c}\mathrm{Re}=700 \\
\mathrm{Re}_{\mathrm{w}}=0,005 \\
\mathrm{Sc}=1650\end{array}$ & $\begin{array}{c}\mathrm{Re}=700 \\
\mathrm{Re}_{\mathrm{w}}=0,0095 \\
\mathrm{Sc}=2500\end{array}$ \\
\hline Elíptico & 1,12 & 1,17 & 1,34 \\
\hline Parabólico & 1,11 & 1,15 & 1,29 \\
\hline Dif. percentual & $1 \%$ & $2 \%$ & $4 \%$ \\
\hline
\end{tabular}


Na Tabela 5 e Tabela 6 temos os valores do perfil de concentração, C, na saída do tubo, os quais estão representados da Figura 12 até Figura 17. A diferença percentual na saída do tubo, para cada modelo (equação convectiva-difusiva elíptica e parabólica) varia de 1\% até $5 \%$, onde "C", na saída do tubo, é sempre maior no modelo elíptico. O termo difusivo axial, $\partial^{2} \mathrm{C} / \partial^{2} \mathrm{X}$, que diferencia o modelo elíptico do parabólico, tem influência, no máximo de $5 \%$, no comportamento do perfil de concentração.

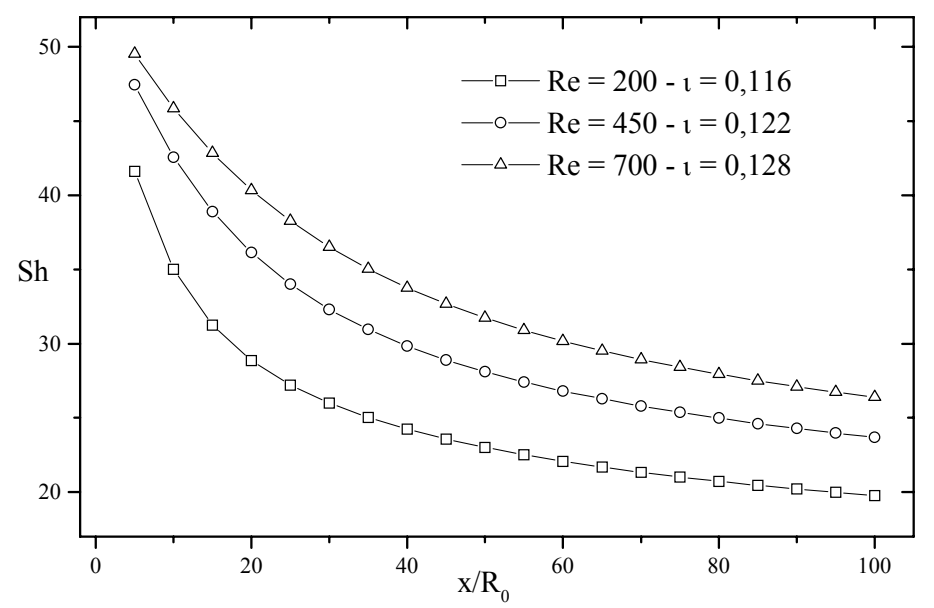

Figura 18. (Equação convectiva-difusiva elíptica) Efeito do número de Reynolds axial sobre o perfil do número de Sherwood local para $\mathrm{Sc}=2500, \mathrm{Re}_{\mathrm{w}}=0,005, \mathrm{R}_{0}=3,010^{-3} \mathrm{~m}, \mathrm{~N}=35$.

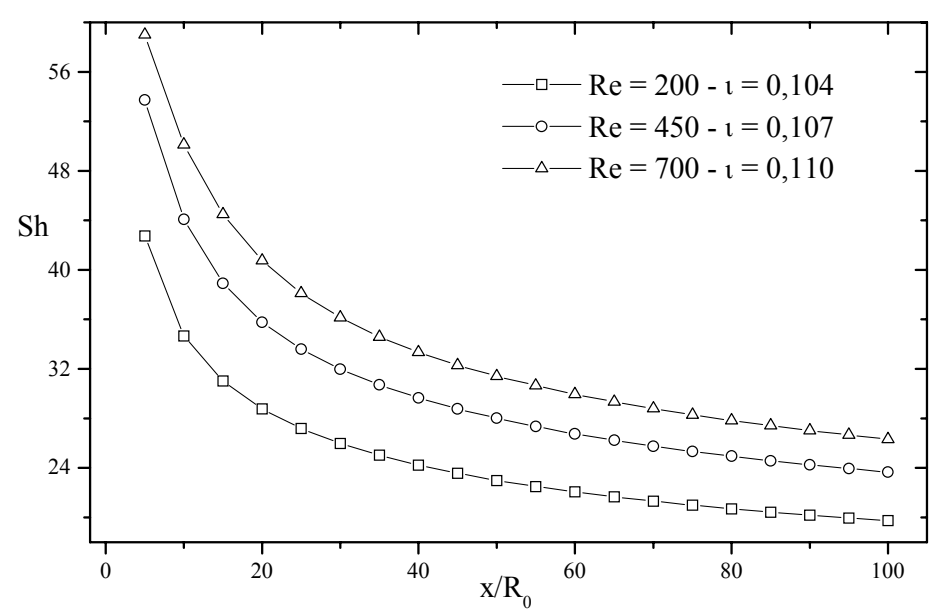

Figura 19. (Equação convectiva-difusiva parabólica) Efeito do número de Reynolds axial sobre o perfil do número de Sherwood local para $\mathrm{Sc}=2500, \mathrm{Re}_{\mathrm{w}}=0,005, \mathrm{~N}=46$ e $\mathrm{R}_{0}=3,010^{-3} \mathrm{~m}$.

Vemos na Figura 18 e Figura 19 os resultados para o número de Sherwood, Sh, em função da posição axial adimensional $\left(\mathrm{x} / \mathrm{R}_{0}\right)$, referentes à equação convectiva-difusiva elíptica e equação convectiva-difusiva parabólica. O comportamento destes perfis com relação às 
respectivas variações do adimensional "Re" está de acordo com a análise feita na $1^{a}$ linha da Tabela 2.

Na Figura 20 e na Figura 21 apresentamos os resultados para o número de Sherwood, Sh, em função da posição axial adimensional $\left(\mathrm{x} / \mathrm{R}_{0}\right)$, referentes à equação convectiva-difusiva elíptica e equação convectiva-difusiva parabólica. O comportamento destes perfís com relação às respectivas variações do adimensional " $\mathrm{Re}_{\mathrm{w}}$ " está de acordo com a análise feita na $3^{\mathrm{a}}$ linha da Tabela 2.

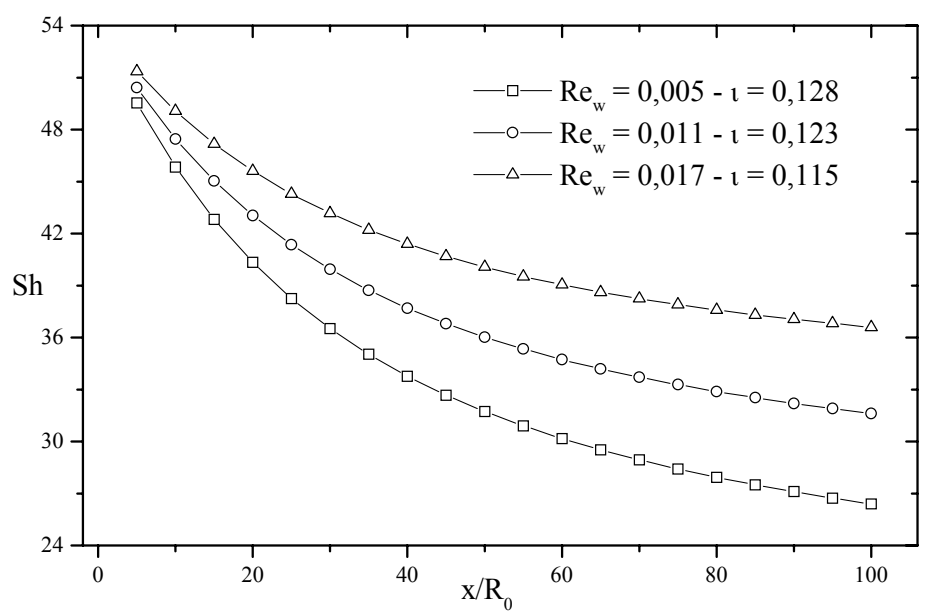

Figura 20. (Equação convectiva-difusiva elíptica) Efeito do número de Reynolds de permeação sobre o perfil do número de Sherwood local para $\mathrm{Sc}=2500, \mathrm{Re}=700, \mathrm{R}_{0}=3,010^{-3} \mathrm{~m}, \mathrm{~N}=35$.

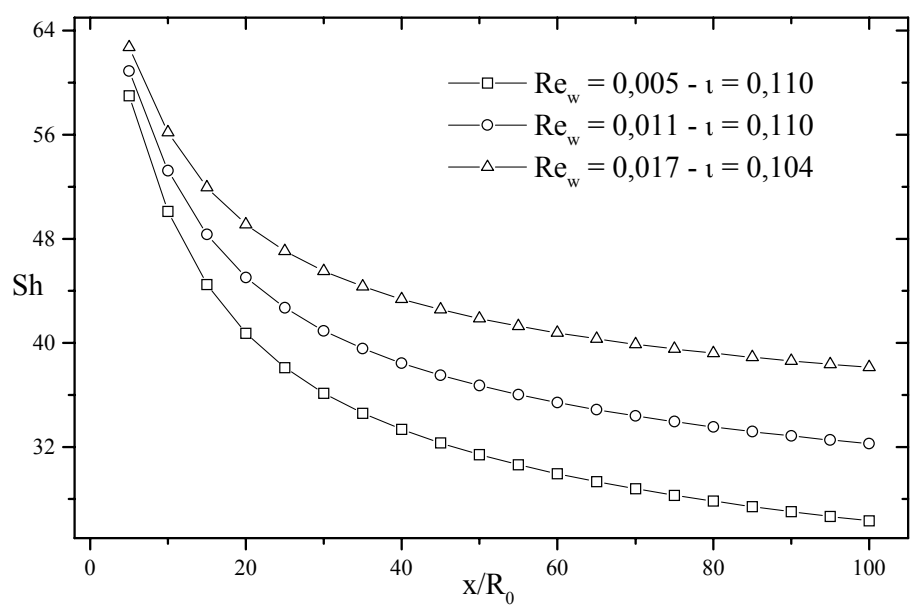

Figura 21. (Equação convectiva-difusiva parabólica) Efeito do número de Reynolds de permeação sobre o perfil do número de Sherwood local para $\mathrm{Sc}=2500, \mathrm{Re}=700, \mathrm{R}_{0}=3,010^{-3} \mathrm{~m}, \mathrm{~N}=46$. 


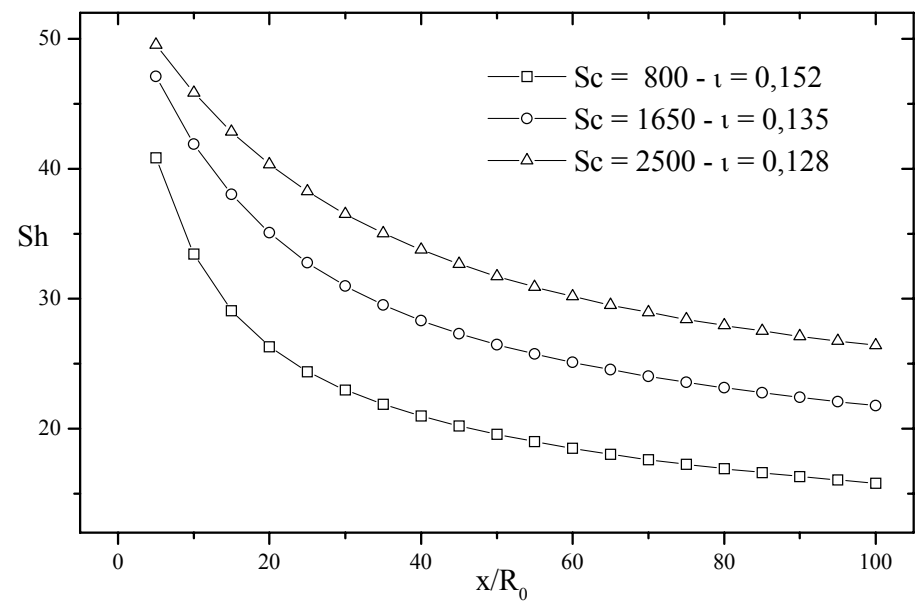

Figura 22. (Equação convectiva-difusiva elíptica) Efeito do número de Schmidt sobre o perfil do número de Sherwood local para $\mathrm{Re}=700, \mathrm{Re}_{\mathrm{w}}=0,005, \mathrm{R}_{0}=3,010^{-3} \mathrm{~m}, \mathrm{~N}=35$.

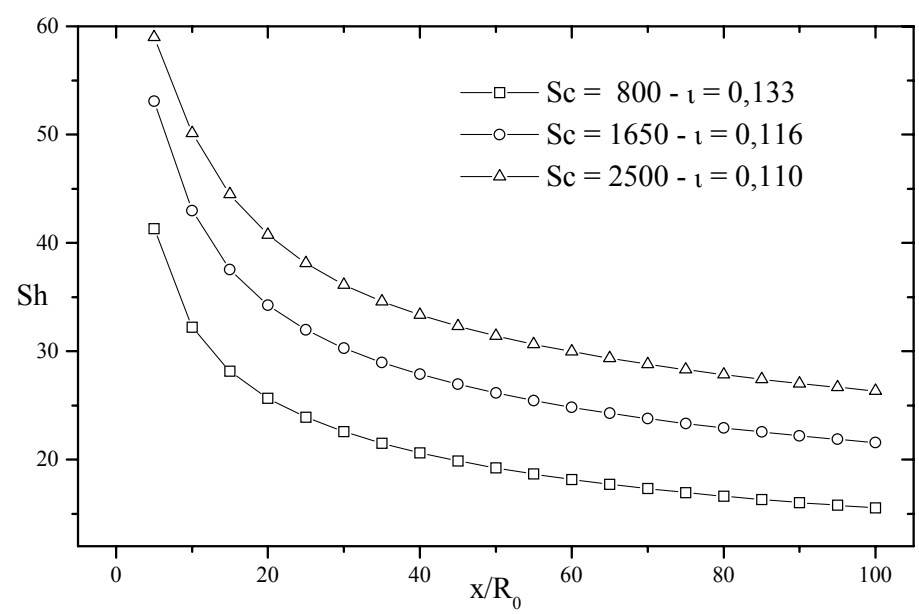

Figura 23. (Equação convectiva-difusiva parabólica) Efeito do número de Schmidt sobre o perfil do número de Sherwood local para $\mathrm{Re}=700, \mathrm{Re}_{\mathrm{w}}=0,005, \mathrm{R}_{0}=3,010^{-3} \mathrm{~m}, \mathrm{~N}=46$.

Vemos na Figura 22 e Figura 23 os resultados para o número de Sherwood, Sh, em função da posição axial adimensional $\left(\mathrm{x} / \mathrm{R}_{0}\right)$, referentes à equação convectiva-difusiva elíptica e equação convectiva-difusiva parabólica. O comportamento destes perfís com relação às respectivas variações do adimensional "Sc" está de acordo com a análise feita na $2^{\text {a }}$ linha da Tabela 2. 
Tabela 7. Resumo das comparações do adimensional de Sherwood, na saída do tubo, $L / R_{0}=100$, entre os modelos elíptico e parabólico. Representação da diferença percentual, onde o sinal negativo indica que o valor do modelo paróbolico é maior.

\begin{tabular}{ccccc}
\cline { 2 - 5 } Modelo & $\begin{array}{c}\mathrm{Re}=700 \\
\mathrm{Re}_{\mathrm{w}}=0,014 \\
\mathrm{Sc}=2500\end{array}$ & $\begin{array}{c}\mathrm{Re}=200 \\
\mathrm{Re}_{\mathrm{w}}=0,005 \\
\mathrm{Sc}=2500\end{array}$ & $\begin{array}{c}\mathrm{Re}=450 \\
\mathrm{Re}_{\mathrm{w}}=0,005 \\
\mathrm{Sc}=2500\end{array}$ & $\begin{array}{c}\mathrm{Re}=700 \\
\mathrm{Re}_{\mathrm{w}}=0,005 \\
\mathrm{Sc}=2500\end{array}$ \\
\hline Elíptico & 36,60 & 19,81 & 23,75 & 26,45 \\
\hline Parabólico & 38,13 & 19,74 & 23,65 & 26,33 \\
\hline Dif. percentual & $-4 \%$ & $0,4 \%$ & $0,4 \%$ & $0,4 \%$ \\
\hline
\end{tabular}

Tabela 8. Continuação do resumo das comparações do adimensional de Sherwood, na saída do tubo, $\mathrm{L} / \mathrm{R}_{0}=100$, entre os modelos elíptico e parabólico. Representação da diferença percentual, onde o sinal negativo indica que o valor do modelo parabólico é maior.

\begin{tabular}{lccc}
\cline { 2 - 4 } Modelo & \multicolumn{3}{c}{$\mathrm{Sh}$} \\
\cline { 2 - 4 } & $\begin{array}{c}\mathrm{Re}=700 \\
\mathrm{Re}_{\mathrm{w}}=0,005 \\
\mathrm{Sc}=800\end{array}$ & $\begin{array}{c}\mathrm{Re}=700 \\
\mathrm{Re}_{\mathrm{w}}=0,005 \\
\mathrm{Sc}=1650\end{array}$ & $\begin{array}{c}\mathrm{Re}=700 \\
\mathrm{Re}_{\mathrm{w}}=0,0095 \\
\mathrm{Sc}=2500\end{array}$ \\
\hline Elíptico & 15,80 & 21,78 & 31,62 \\
\hline Parabólico & 15,53 & 21,56 & 32,25 \\
\hline Dif. percentual & $2 \%$ & $1 \%$ & $-2 \%$ \\
\hline
\end{tabular}

Na Tabela 7 e Tabela 8 temos os valores do adimensional de Sherwood, Sh, na saída do tubo, os quais estão representados da Figura 18 até Figura 23. A diferença percentual na saída do tubo, para cada modelo (equação convectiva-difusiva elíptica e parabólica) varia de $-4 \%$ até $+2 \%$, onde o sinal negativo indica que "Sh" é maior para o modelo parabólico. $\mathrm{O}$ termo difusivo axial, $\partial^{2} \mathrm{C} / \partial^{2} \mathrm{X}$, que diferencia o modelo elíptico do parabólico, tem influência, no máximo de 4\%, no comportamento do adimensional de Sherwood.

\subsubsection{Comparações do Adimensional de Sherwood com Outros Resultados da LITERATURA}

Nesta seção compararemos o adimensional de Sherwood, Sh, provindo de resultados de simulações da GITT com outros da literatura. Na literatura "Sh" é dado através de correlações, análogos aos que foram apresentados na Seção (2.1.10). Para este fim, desenvolvemos no Apêndice (4) duas correlações de Gilland-Sherwood, Equação (145) e 
Equação (146), a partir de simulações, via GITT, de cada modelo de interesse deste trabalho, ou seja, para:

- equação convectiva-difusiva parabólica

$$
\mathrm{Sh}=0,7438(\operatorname{ReSc} / \mathrm{X})^{0,3674}\left(1+1751,9133 \operatorname{Re}^{-1,2132} \operatorname{Sc}^{1,2695} \operatorname{Re}_{\mathrm{w}}^{2,5607}\right)
$$

- equação convectiva-difusiva elíptica

$$
\mathrm{Sh}=0,9938(\operatorname{ReSc} / \mathrm{X})^{0,3357}\left(1+2,8546 \mathrm{Re}^{-0,4500} \mathrm{Sc}^{0,6723} \operatorname{Re}_{\mathrm{w}}^{1,1035}\right)
$$

No trabalho de Damak et al. (2004) é modelado o problema físico análogo ao realizado no presente trabalho, isto é, utiliza a equação convectiva-difusiva elíptica, contudo os resultados foram obtidos via diferenças finitas. No mesmo tem-se determinado uma expressão matemática para a correlação de Gilland-Sherwood, dada por:

$$
\operatorname{Sh}=1,23(\operatorname{ReSc} / \mathrm{X})^{0,33}\left(1+0,01 \operatorname{Re}^{-0,125} \operatorname{Sc}^{1,055} \operatorname{Re}_{\mathrm{w}}^{1,132}\right)
$$

Uma outra expressão importante é a correlação de Leveque (ZEMAN e ZYDNEY, 1996):

$$
\mathrm{Sh}=1,62(\operatorname{ReSc} / \mathrm{X})^{0,33}
$$

Na Figura 24 e na Figura 25 mostramos as comparações para "Sh", dadas pelas Equações (104) e (105), em função da posição axial, x/R $\mathrm{R}_{0}$. Da Figura 26 até Figura 29 mostramos as comparações de "Sh" em função da posição axial, $\mathrm{x} / \mathrm{R}_{0}$, dadas pelas Equações (104), (105), (106) e (107), variando os adimensionais "Re", "Re $\mathrm{w}_{\mathrm{w}}$ " e "Sc". Observamos que a correlação de Leveque (Equações (107)) não foi desenvolvida para filtração tangencial, mas para transferência de massa em sistemas impermeáveis, daí a ocorrência das altas diferenças percentuais obtidas nos parágrafos abaixo.

Na saída do tubo, a Figura 24 mostra que o resultado da Equação (104) é menor, por uma diferença percentual de aproximadamente 5\%, daquele resultado dado pela Equação (105). 


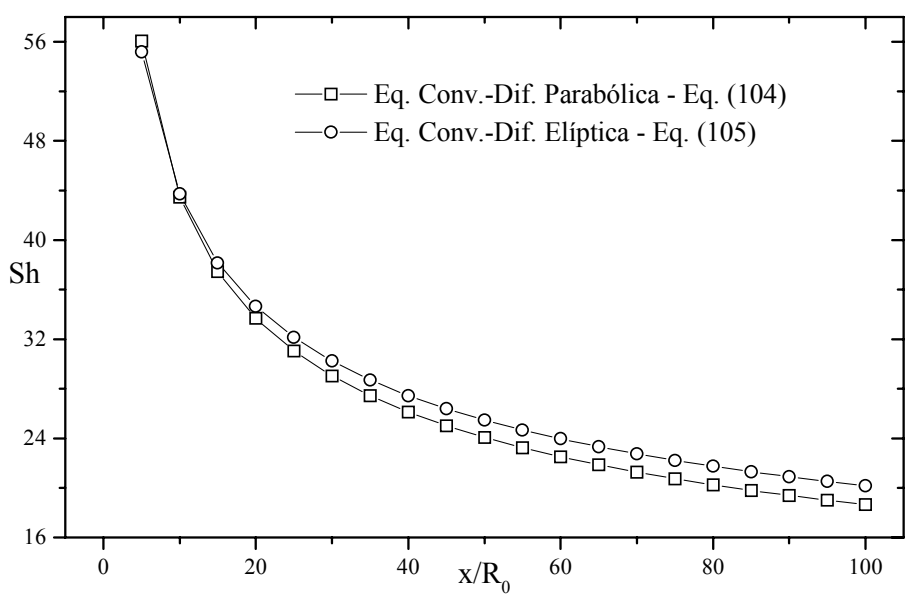

Figura 24. Comparação entre as correlações de Gilland-Sherwood, sendo $\mathrm{R}_{0}=3,010^{-3} \mathrm{~m}, \mathrm{Re}=700, \mathrm{Sc}=800$, $\operatorname{Re}_{\mathrm{w}}=0,014$.

A Figura 25 mostra que, na saída do tubo, o resultado da Equação (104) é menor, por uma diferença percentual de aproximadamente $9 \%$, daquele resultado dado pela Equação (105).

As diferenças existentes entre os resultados da correlação de Sherwood, a partir da solução das equações convectiva-difusiva parabólica e elíptica, respectivamente vistas na Figura 24 e Figura 25, são causadas pelo termo difusivo axial existente na equação convectiva-difusiva elíptica, contudo negligenciado no problema parabólico.

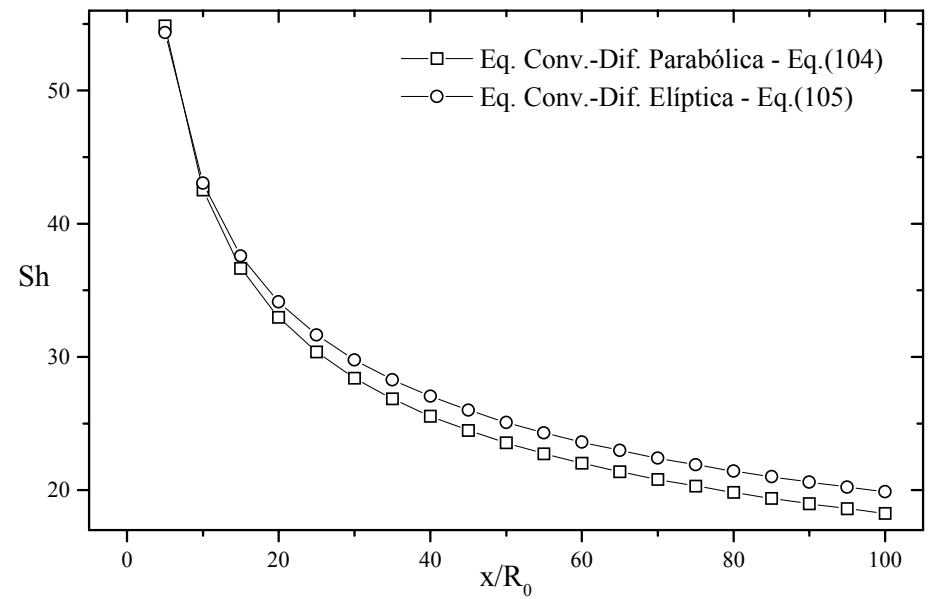

Figura 25. Comparação entre as correlações de Gilland-Sherwood, sendo $\mathrm{R}_{0}=3,010^{-3} \mathrm{~m}, \mathrm{Re}=200, \mathrm{Sc}=2500$, $\operatorname{Re}_{\mathrm{w}}=0,005$. 


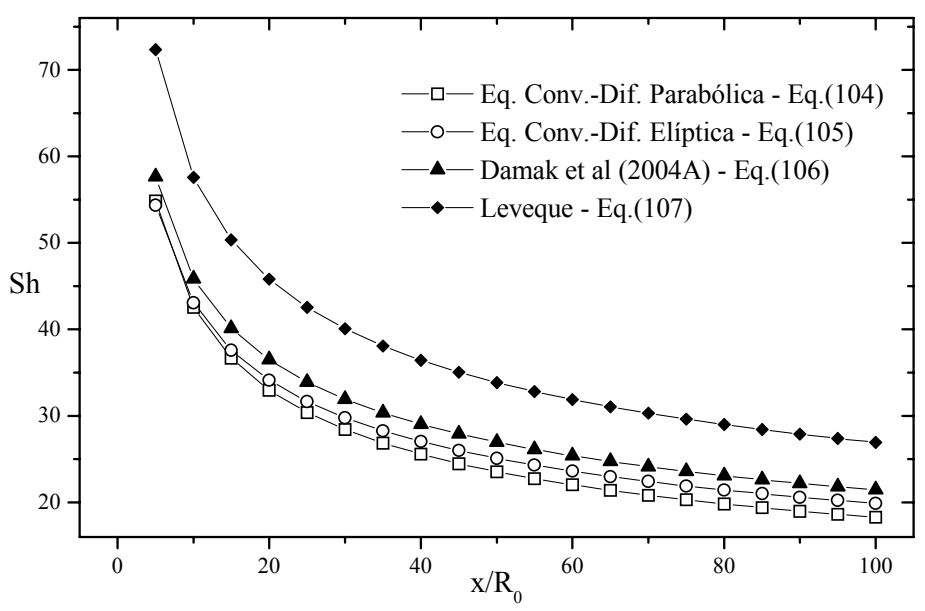

Figura 26. Comparações entre as correlações de Gilland-Sherwood, sendo $\mathrm{R}_{0}=3,010^{-3} \mathrm{~m}, \mathrm{Re}=200, \mathrm{Sc}=2500$, $\mathrm{Re}_{\mathrm{w}}=0,005$.

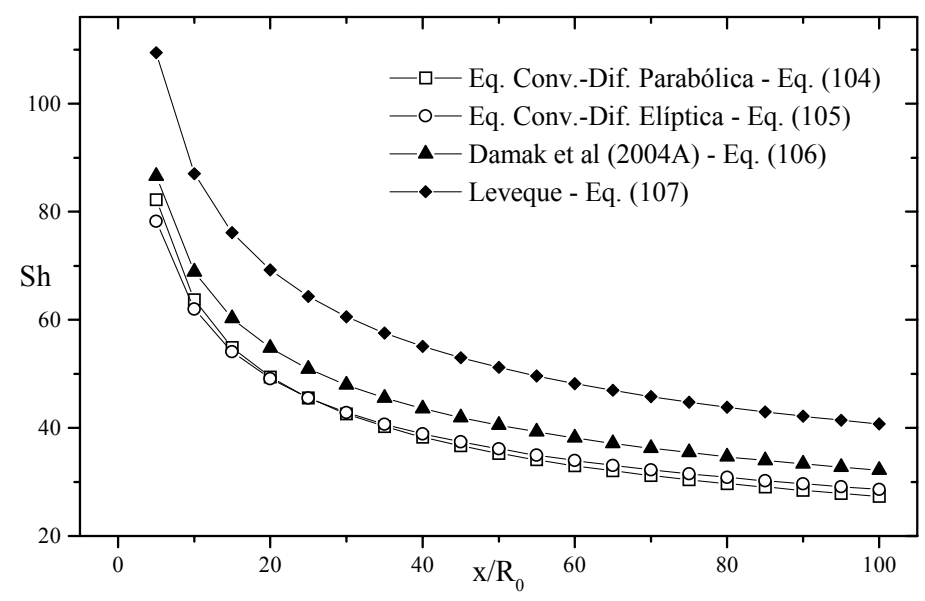

Figura 27. Comparações entre as correlações de Gilland-Sherwood, sendo $\mathrm{R}_{0}=3,010^{-3} \mathrm{~m}, \mathrm{Re}=700, \mathrm{Sc}=2500$, $\mathrm{Re}_{\mathrm{w}}=0,005$.

Na Figura 26 vemos que, na saída do tubo, o resultado da Equação (104) é menor, por uma diferença percentual de aproximadamente $17 \%$, daquele resultado de Damak et al. (2004A) e por uma diferença percentual de aproximadamente $47 \%$ menor que o da correlação de Leveque. Além disso, o resultado da Equação (105) é menor, por uma diferença percentual de aproximadamente 8\%, daquele resultado de Damak et al. (2004A) e por uma diferença percentual de aproximadamente $35 \%$ menor que o da correlação de Leveque.

Na Figura 27 o resultado da Equação (104) é menor, por uma diferença percentual de aproximadamente 17\%, daquele resultado de Damak et al. (2004A) e por uma diferença percentual de aproximadamente $49 \%$ menor que o da correlação de Leveque. Esta figura mostra ainda que o resultado da Equação (105) é menor, por uma diferença percentual de aproximadamente $12 \%$, daquele resultado de Damak et al. (2004A) e por uma diferença 
percentual de aproximadamente $44 \%$ menor que o da correlação de Leveque.

A Figura 28 mostra que o resultado da Equação (104) é menor, por uma diferença percentual de aproximadamente 6\%, daquele resultado de Damak et al. (2004A) e por uma diferença percentual de aproximadamente $23 \%$ menor que o da correlação de Leveque. Esta figura mostra ainda que o resultado da Equação (105) é menor, por uma diferença percentual de aproximadamente 5\%, daquele resultado de Damak et al. (2004A) e por uma diferença percentual de aproximadamente $22 \%$ menor que o da correlação de Leveque.

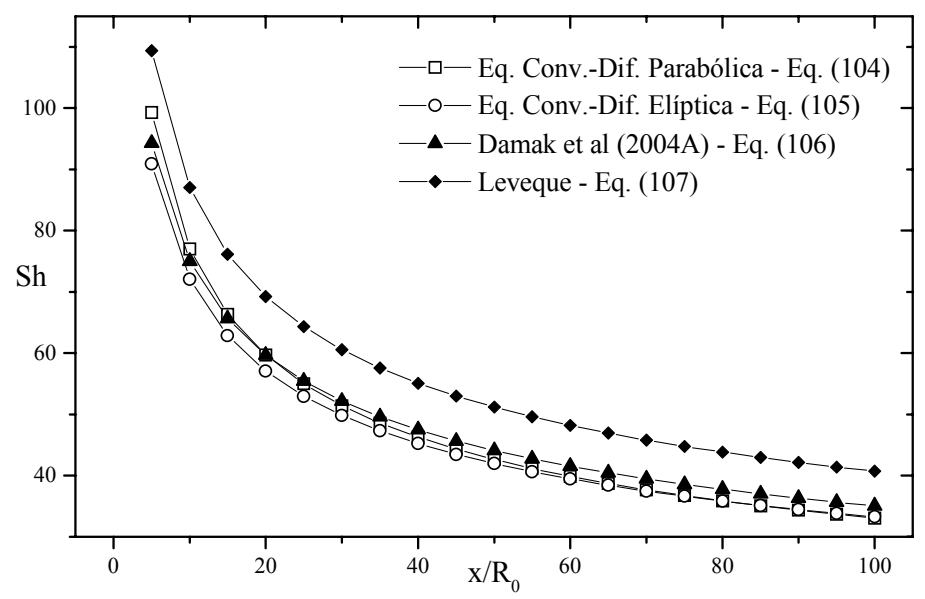

Figura 28. Comparações entre as correlações de Gilland-Sherwood, sendo $\mathrm{R}_{0}=3,010^{-3} \mathrm{~m}, \mathrm{Re}=700, \mathrm{Sc}=2500$, $\operatorname{Re}_{\mathrm{w}}=0.014$.

Na Figura 29 o resultado da Equação (104) é menor, por uma diferença percentual de aproximadamente 18\%, daquele resultado de Damak et al. (2004A) e por uma diferença percentual de aproximadamente 50\% menor que o da correlação de Leveque. Esta figura mostra ainda que o resultado da Equação (105) é maior, por uma diferença percentual de aproximadamente $9 \%$, daquele resultado de Damak et al. (2004A) e por uma diferença percentual de aproximadamente $38 \%$ menor que o da correlação de Leveque.

As diferenças existentes nos resultados da Figura 26 até a Figura 29 podem ter sido causadas por diferentes formulações matemáticas consideradas em cada modelo. 


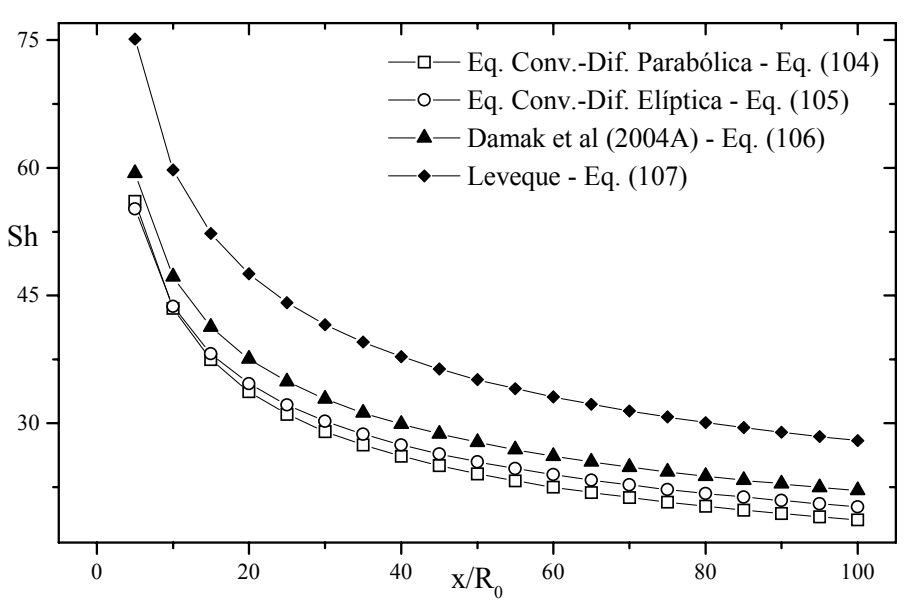

Figura 29. Comparações entre as correlações de Gilland-Sherwood, sendo $\mathrm{R}_{0}=3,010^{-3} \mathrm{~m}, \mathrm{Re}=700, \mathrm{Sc}=800$, $\mathrm{Re}_{\mathrm{w}}=0,014$.

\subsubsection{Comparações do Fluxo Transmembrana e do Perfil de Concentração com OUTROS RESUltados DA Literatura}

Na Figura 30 e na Figura 31 mostramos as comparações do fluxo transmembrana local, $\mathrm{J}$, em função da posição axial, $\mathrm{x} / \mathrm{R}_{0}$, com os resultados de Paris et al. (2002). Os parâmetros que representam o problema físico encontram-se indicados em cada figura.

A Figura 30 e Figura 31 mostram as comparações do fluxo transmembrana local e da espessura da camada limite de concentração do trabalho de Paris et al. (2002) com os resultados das soluções analítico-numéricas, via GITT, da equação convectiva-difusiva parabólica e elíptica, respectivamente. Os parâmetros utilizados na simulação foram (Tabela 19, Apêndice (5)): $\operatorname{Re}=648, \mathrm{Re}_{\mathrm{w}}=0,004, \mathrm{Sc}=2500$. O tubo permeável utilizado neste trabalho experimental tem comprimento $L=1,2 \mathrm{~m}$ e raio $\mathrm{R}_{0}=0,003 \mathrm{~m}$ e neste processo considerou-se $\rho=1,010^{3} \mathrm{~kg} \mathrm{~m}^{-3}$ e $\mu=1,010^{-3} \mathrm{~kg} \mathrm{~m}^{-1} \mathrm{~s}^{-1}$.

Vemos na Figura 30 que os resultados provindos da equação convectiva-difusiva parabólica e elíptica, respectivamente, são menores, por uma diferença percentual de aproximadamente $17 \%$ e $0,1 \%$ na saída do tubo e $28 \%$ e $9 \%$ na posição axial x/R $R_{0}=200$, daquele resultado de Paris et al. (2002). Os valores para o fluxo transmembrana local, J, da 
simulação do modelo elíptico têm maior aproximação ao resultado numérico da literatura. Contudo, no trabalho de Paris et al. (2002) é utilizado como modelo a equação convectivadifusiva parabólica e o fluxo transmembrana local são avaliados a partir do modelo da resistência em série. No presente trabalho, o fluxo é calculado a partir do modelo gel polarizado. Observa-se na Figura 30 as taxas assintóticas, 1, e o fluxo transmembrana médio para cada um dos problemas parabólico, $\mathrm{J}_{\mathrm{m}}=1,3410^{-6} \mathrm{~m} / \mathrm{s}$ e problema elíptico, $\mathrm{J}_{\mathrm{m}}=1,3810^{-6}$ $\mathrm{m} / \mathrm{s}$, e estes resultados estão bem próximos do valor do fluxo médio experimental (Paris et al., (2002)), $\mathrm{J}_{\mathrm{m}, \mathrm{exp}}=1,2410^{-6} \mathrm{~m} / \mathrm{s}$. As taxas assintóticas obtidas, $\mathrm{l}=0,106$ e $\mathrm{\imath}=0,118$, são menores que a taxa assintótica máxima, $1=0,226$, satisfazendo o critério discutido na Seção (3.3.3).

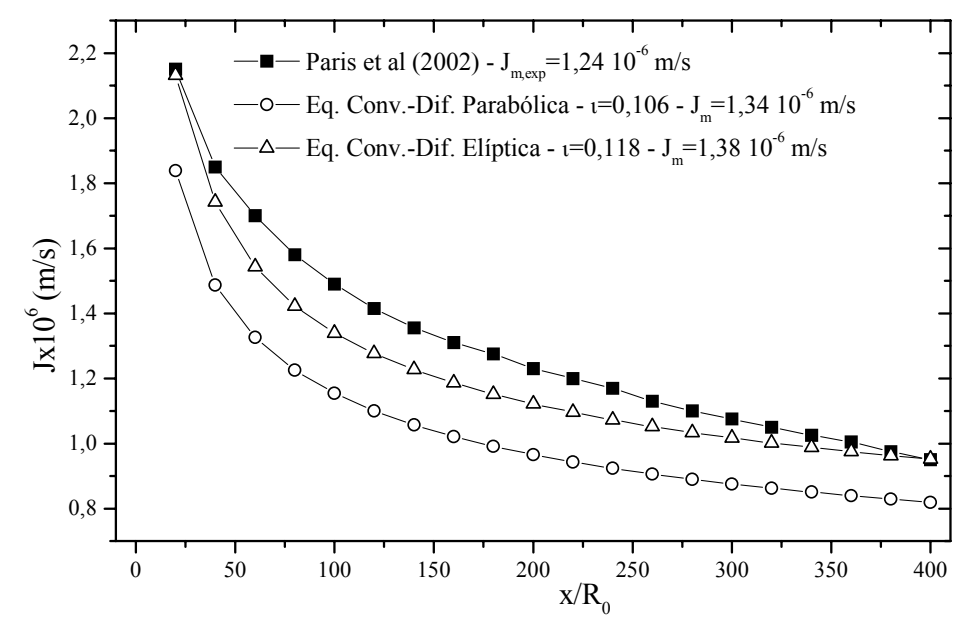

Figura 30. Comparação entre os resultados deste trabalho com outro numérico da literatura para o fluxo transmembrana local, com $\mathrm{c}_{0}=8 \mathrm{~g} / \mathrm{l}, \Delta \mathrm{p}=4,910^{5} \mathrm{~Pa}, \mathrm{u}_{0}=0,216 \mathrm{~m} / \mathrm{s}, \mathrm{R}_{\mathrm{m}}+\mathrm{R}_{\mathrm{e}}=2,6710^{10} \mathrm{~Pa} \mathrm{~s} \mathrm{~m}^{-1}, \phi=6,8710^{5} \mathrm{~s} \mathrm{~m}^{-1}$,

$$
\mathrm{v}_{\mathrm{w}}=1,3510^{-6} \mathrm{~m} \mathrm{~s}^{-1} \mathrm{e}_{\iota_{\max }}=0,226 \text {. }
$$

A simulação realizada para a geração da Figura 30, possibilita também a construção dos resultados da Figura 31, que mostra a comparação da espessura da camada limite de concentração entre os resultados deste trabalho e do resultado de Paris et al. (2002). Os resultados provindos da equação convectiva-difusiva parabólica e elíptica, respectivamente, são maiores, por uma diferença percentual de aproximadamente $11 \%$ e $25 \%$ na saída do tubo, 
daquele resultado de Paris et al. (2002).

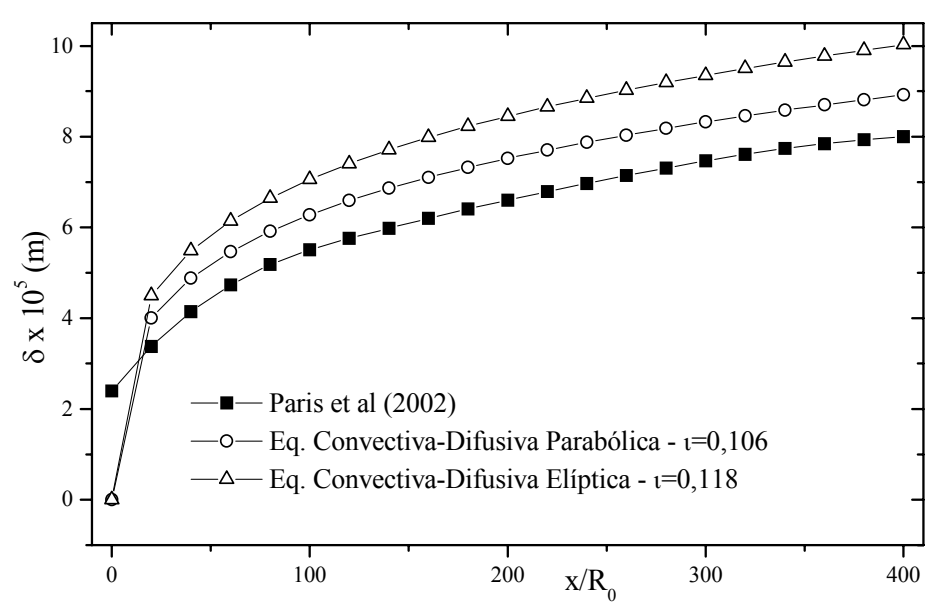

Figura 31. Comparação entre os resultados deste trabalho com outro numérico da literatura para a espessura da camada limite de concentração, com $\mathrm{c}_{0}=8 \mathrm{~g} \mathrm{l}^{-1}, \Delta \mathrm{p}=4,910^{5} \mathrm{~Pa}, \mathrm{R}_{\mathrm{m}}+\mathrm{R}_{\mathrm{e}}=2,6710^{10} \mathrm{~Pa} \mathrm{~s} \mathrm{~m}^{-1}, \mathrm{v}_{\mathrm{w}}=1,3510^{-6} \mathrm{~m} \mathrm{~s}^{-1}$, $\phi=6,8710^{5} \mathrm{~s} \mathrm{~m}^{-1}, \mathrm{u}_{0}=0,216 \mathrm{~m} \mathrm{~s}^{-1} \mathrm{e} \iota_{\max }=0,226$.

As diferenças existentes nos resultados na Figura 30 e na Figura 31 podem ter sido causadas por diferentes formulações matemáticas consideradas em cada modelo.

Para as simulações dos resultados via GITT, mostrados na Figura 32 e na Figura 33, utilizamos o raio do poro $\mathrm{r}_{\mathrm{po}}=3,210^{-6} \mathrm{~m}$, a espessura da membrana $\delta_{\mathrm{m}}=1,010^{-6} \mathrm{~m}$ e a lei de Poiseuille (Equação (3)). Outros parâmetros utilizados por Silva (2008) estão indicados na legenda de cada figura. Para determinação das taxas assintóticas, utilizamos a metodologia da Seção (3.3.2) associada, respectivamente, às equações convectiva-difusiva elíptica e parabólica.

Vemos na Figura 32 que os resultados para o perfil de concentração provindos da equação convectiva-difusiva elíptica e parabólica, respectivamente, são menores, por uma diferença percentual de aproximadamente $5 \%$ e $8 \%$ próximos à saída do tubo, daquele resultado de Silva (2008). 


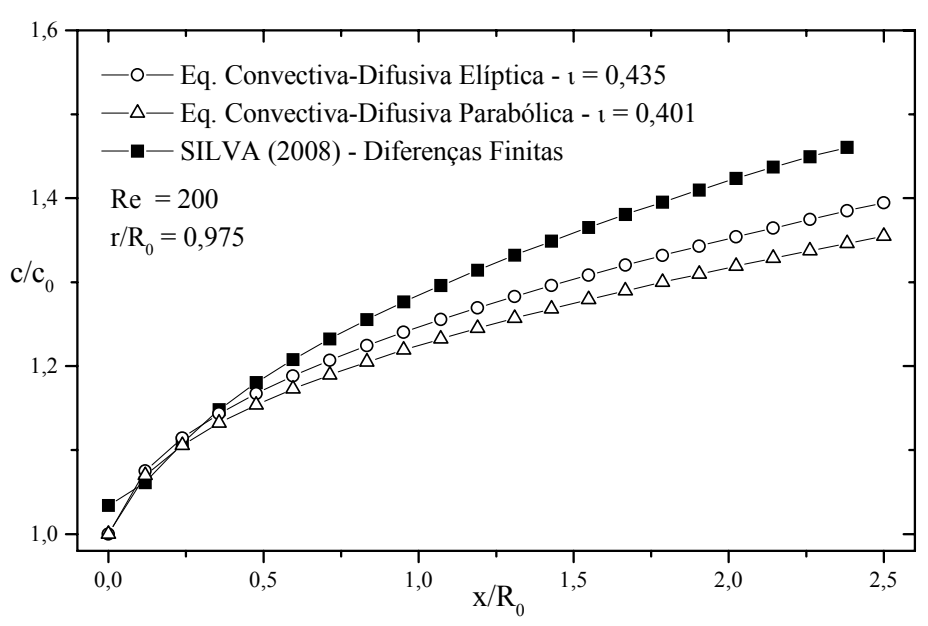

Figura 32. Comparação entre os resultados deste trabalho com outro numérico para o perfil de concentração, com $\Delta \mathrm{p}=1,010^{5} \mathrm{~Pa}, \mathrm{R}_{\mathrm{m}}=2,810^{9} \mathrm{~Pa} \mathrm{~s} \mathrm{~m}^{-1}, \mathrm{R}_{\mathrm{e}}=0,110^{9} \mathrm{~Pa} \mathrm{~s} \mathrm{~m}^{-1}, \phi=0,9610^{5} \mathrm{~s} \mathrm{~m}^{-1}, \mathrm{R}_{0}=1,510^{-2} \mathrm{~m}, \mathrm{Sc}=14$, $\operatorname{Re}_{\mathrm{w}}=0,52, \mu=10^{-3} \mathrm{~kg} \mathrm{~m}^{-1} \mathrm{~s}^{-1} \mathrm{e} \rho=10^{3} \mathrm{~kg} \mathrm{~m}^{-1}$.

Na Figura 33 é mostrado que os resultados para o perfil de concentração provindos das equações convectiva-difusiva elíptica e parabólica, respectivamente, são menores, por uma diferença percentual de aproximadamente $11 \%$ e $15 \%$ próximos à saída do tubo, daquele resultado de Silva (2008). Para determinação das taxas assintóticas, e $\imath=0,136$, utilizamos a metodologia da Seção (3.3.2) associada, respectivamente, às equações convectiva-difusiva elíptica e parabólica, cuja taxa assintótica máxima atingiu $\imath=0,376$, satisfazendo assim o critério discutido na Seção (3.3.3).

As diferenças existentes nos resultados na Figura 32 e na Figura 33 podem ter sido causadas por diferentes formulações matemáticas consideradas em cada modelo. 


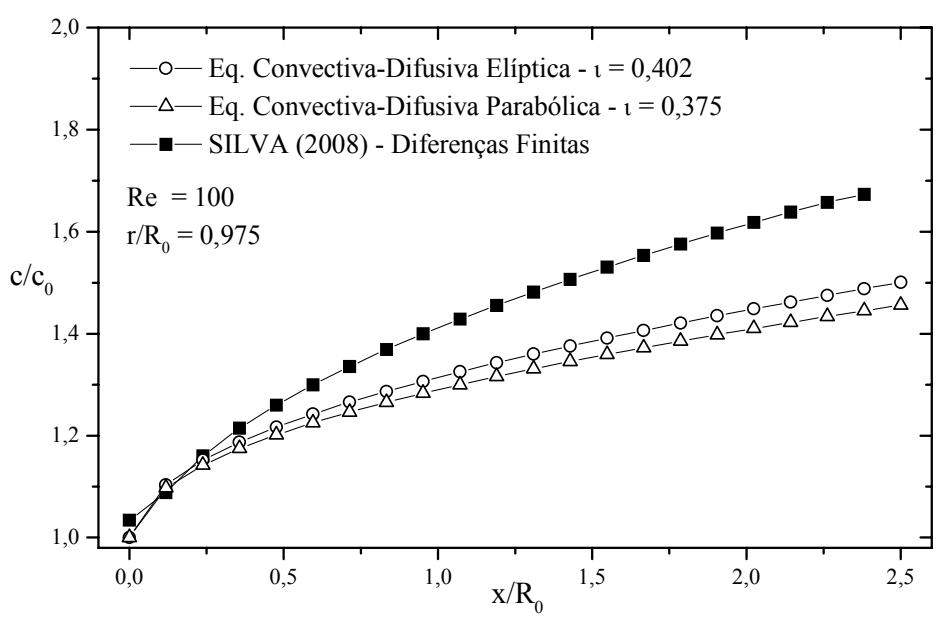

Figura 33. Comparação entre os resultados deste trabalho com outro numérico para o perfil de concentração, com $\Delta \mathrm{p}=1,010^{5} \mathrm{~Pa}, \mathrm{R}_{\mathrm{m}}=2,810^{9} \mathrm{~Pa} \mathrm{~s} \mathrm{~m}^{-1}, \mathrm{R}_{\mathrm{e}}=0,110^{9} \mathrm{~Pa} \mathrm{~s} \mathrm{~m}^{-1}, \phi=0,9610^{5} \mathrm{~s} \mathrm{~m}^{-1}, \mathrm{R}_{0}=1,510^{-2} \mathrm{~m}, \mu=10^{-3} \mathrm{~kg} \mathrm{~m}^{-1} \mathrm{~s}^{-1}$,

$$
\rho=10^{3} \mathrm{~kg} \mathrm{~m}^{-1}, \mathrm{Sc}=14 \mathrm{e} \mathrm{Re}_{\mathrm{w}}=0,52 \text {. }
$$

\subsubsection{ANÁlise da ESPESSURa da CAMAda Limite de CONCENTRAÇão}

Nesta seção, além da análise dos resultados da concentração polarizada e da espessura da camada limite de concentração, mostraremos que a Equação (97) pode ser utilizada para avaliarmos a espessura da camada limite de concentração. Estes resultados são gerados a partir da metodologia da Seção (3.3.2), onde neste processo utilizamos à equação convectivadifusiva parabólica (Equação (21)), sendo o erro percentual $\varepsilon=0,01$. Os resultados gerados a partir da formulação GITT reproduzem a solução completamente convergida e estabelecida para $\mathrm{N}$ maior que 46 , com erro relativo global de $10^{-3}$.

Da Figura 34 até a Figura 39 temos o comportamento da espessura da camada limite de concentração e da concentração polarizada, quando variamos os adimensionais: número de Reynolds axial, Re, número de Reynolds radial ou de permeação, $\mathrm{Re}_{\mathrm{w}}$, e número de $\mathrm{Schmidt}$, Sc. A taxa assintótica, 1, também pode ser observada com a variação destes adimensionais. As condições destes resultados estão especificadas em cada figura.

Comparamos a formulação proposta para a espessura da camada limite de concentração 
(Equações (97)) com resultados numéricos obtidos por diversos autores, as quais podem ser vistas na Figura 40 e na Figura 41. As condições destes resultados estão especificadas em cada figura.

Apresentamos na Figura 34 o resultado da espessura da camada limite de concentração, $\delta$, em função da posição axial adimensional, $\mathrm{x} / \mathrm{R}_{0}$. Observamos uma tendência de diminuição da espessura da camada limite de concentração e de aumento da taxa assintótica em função do aumento do número de Reynolds, sendo iguais a 200; 450 e 700. A espessura da camada limite de concentração tem diferença percentual máxima na saída do tubo, aproximadamente de $28 \%$, para $200 \leq \operatorname{Re} \leq 700$.

Quando diminuímos o número de Reynolds, o transporte de partículas de soluto rejeitadas pela membrana não volta rapidamente para a corrente livre e o soluto rejeitado tende a aumentar nas adjacências da membrana. Assim a Figura 35 está de acordo com este fenômeno. Nesta figura vemos que a concentração polarizada tem variação percentual, aproximadamente de $14 \%$ na saída do tubo, para $200 \leq \operatorname{Re} \leq 700$.

Pela Figura 34 e pela Figura 35 podemos concluir que o aumento de "Re" induz uma diminuição máxima de $14 \%$ na concentração polarizada e uma diminuição $28 \%$ na espessura da camada limite de concentração. Pela Equação (103) concluímos que, se aumentarmos “Re", o termo $\left(\operatorname{ReSc}_{\mathrm{Re}}\right)^{-1 / 3}$ diminuirá, e assim a taxa assintótica, 1 , aumentará, para qualquer $1 \leq \mathfrak{l}_{\max }$. Estas tendências estão de acordo com a análise feita na Seção (2.1.4). 


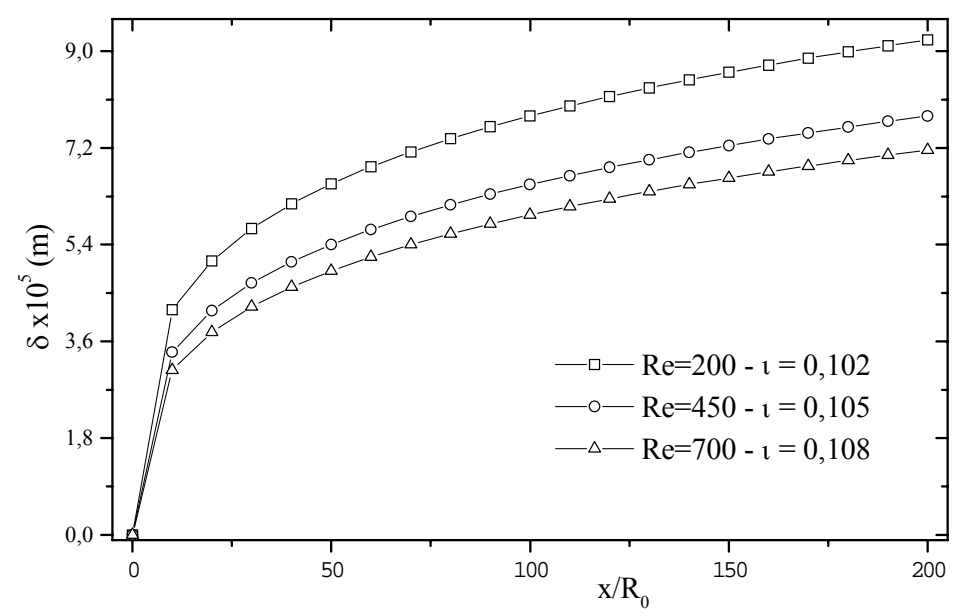

Figura 34. Efeito do número de Reynolds sobre a espessura da camada limite de concentração em função da posição axial adimensional $\left(\mathrm{x} / \mathrm{R}_{0}\right)$, com $\mathrm{R}_{0}=3,010^{-3} \mathrm{~m}, \mathrm{Sc}=2500$ e $\mathrm{Re}_{\mathrm{w}}=0,005$.

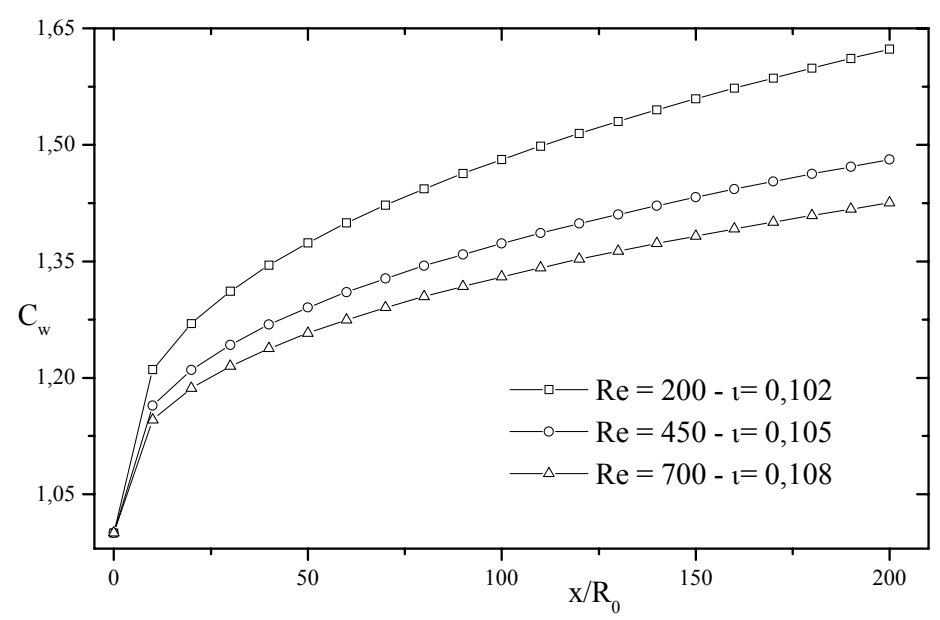

Figura 35. Efeito do número de Reynolds sobre a concentração polarizada em função da posição axial adimensional $\left(\mathrm{x} / \mathrm{R}_{0}\right)$, com $\mathrm{R}_{0}=3,010^{-3} \mathrm{~m}, \mathrm{Re}_{\mathrm{w}}=0,005, \mathrm{Sc}=2500$.

O resultado da espessura da camada limite de concentração, $\delta$, em função da posição axial adimensional, $\mathrm{x} / \mathrm{R}_{0}$, é apresentado na Figura 36. Observamos uma tendência de diminuição da espessura da camada limite de concentração e também diminuição da taxa assintótica em função do aumento do número de Reynolds de permeação, sendo iguais a 0,005, 0,011 e 0,017. Estas tendências estão compatíveis com o que foi discutido no final da Seção (3.3.1). Nesta figura vemos que a espessura da camada limite de concentração tem variação percentual, aproximadamente de $69 \%$ na saída do tubo, para $0,005 \leq \operatorname{Re}_{\mathrm{w}} \leq 0,017$. 
Quando aumentamos o número de Reynolds de permeação, ocorre o aumento na concentração de polarização, $C_{w}$, e dentro do intervalo $0,005 \leq \mathrm{Re}_{\mathrm{w}} \leq 0,017$, esta concentração tem variação percentual máxima de aproximadamente de $76 \%$, o qual pode ser visto na Figura 37.

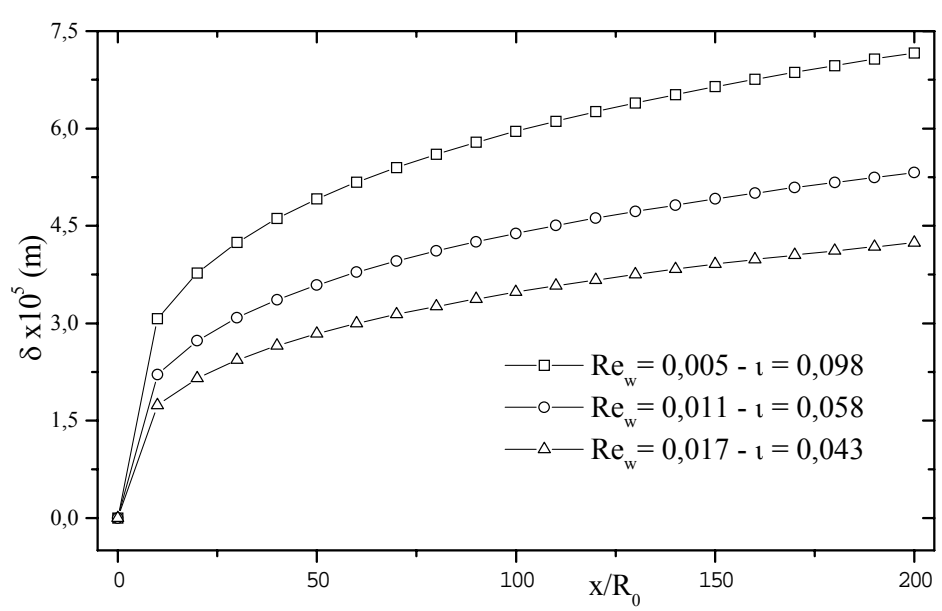

Figura 36. Efeito do número de Reynolds de permeação sobre a espessura da camada limite de concentração em função da posição axial adimensional $\left(\mathrm{x} / \mathrm{R}_{0}\right)$, com $\mathrm{R}_{0}=3,010^{-3} \mathrm{~m}, \mathrm{Re}=700, \mathrm{Sc}=2500$.

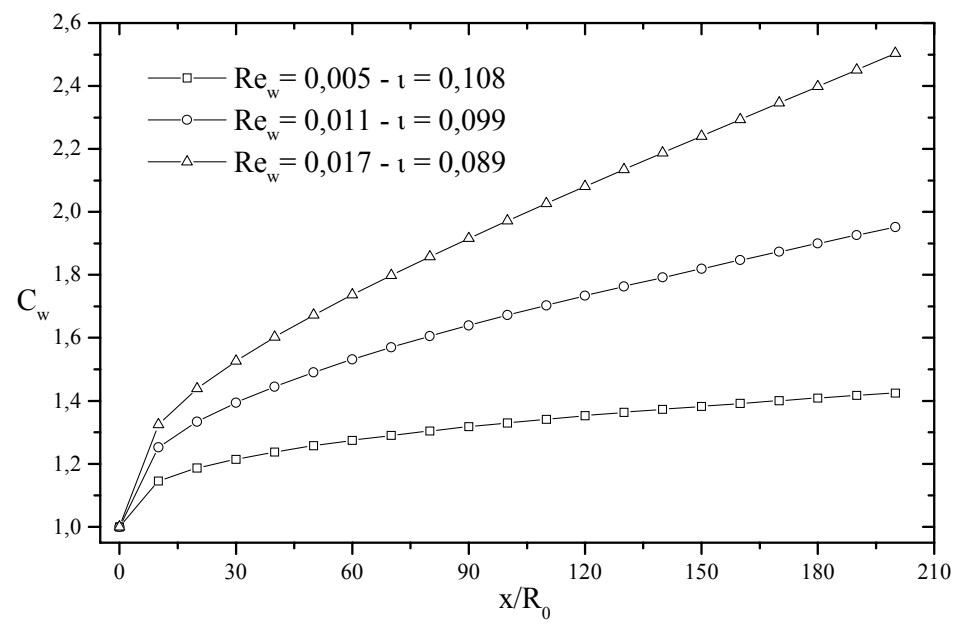

Figura 37. Efeito do número de Reynolds de permeação sobre a concentração polarizada em função da posição axial adimensional $\left(\mathrm{x} / \mathrm{R}_{0}\right)$, com $\mathrm{R}_{0}=3,010^{-3} \mathrm{~m}, \mathrm{Re}=700, \mathrm{Sc}=2500$.

Observando os resultados das Figura 36 e Figura 37 concluímos que o aumento de " $\mathrm{Re}_{\mathrm{w}}$ " promove um aumento máximo de $76 \%$ na concentração polarizada e uma diminuição máxima de 32\% na espessura da camada limite de concentração, e estes resultados estão de 
acordo com a análise feita na Seção (2.1.4). Pela Equação (103), se aumentarmos "Re ${ }_{\mathrm{w}}$ " resultará na diminuição da taxa assintótica, 1 .

Apresentamos na Figura 38 o resultado da espessura da camada limite de concentração, $\delta$, em função da posição axial adimensional, x/R. Observamos uma diminuição da espessura da camada limite de concentração e também diminuição da taxa assintótica em função do aumento do número de Schmidt, sendo estes iguais a 800,1650 e 2500 . Esta tendência é compatível com o que foi discutido no final da Seção (3.3.1). Na Figura 38 vemos que a espessura da camada limite de concentração tem variação percentual máxima aproximadamente de $68 \%$ na saída do tubo, para $800 \leq \mathrm{Sc} \leq 2500$.

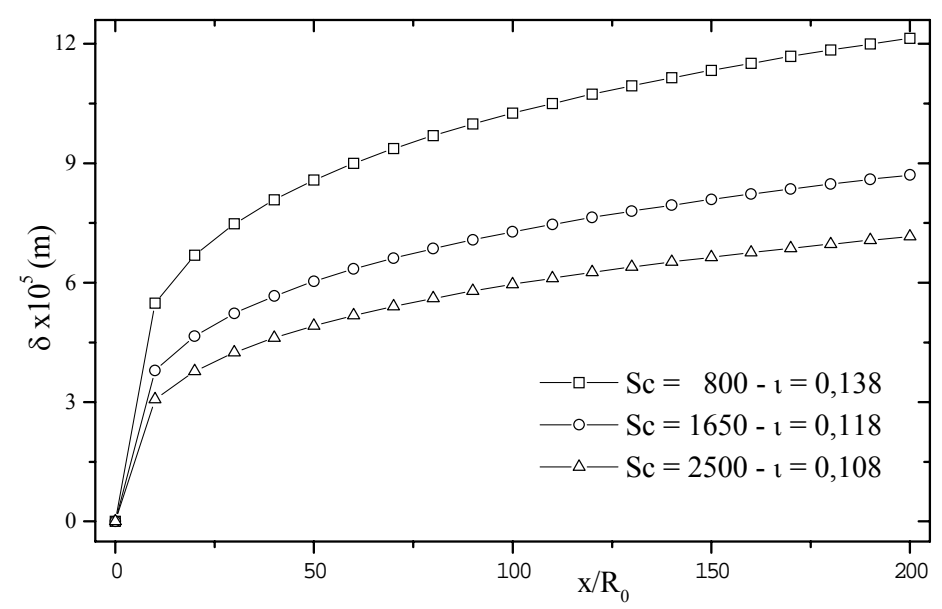

Figura 38. Efeito do número de Schmidt sobre as espessuras das camadas limite de concentração em função da posição axial adimensional $\left(\mathrm{x} / \mathrm{R}_{0}\right)$, com $\mathrm{Re}=700, \mathrm{Re}_{\mathrm{w}}=0,005$ e $\mathrm{R}_{0}=3,010^{-3} \mathrm{~m}$.

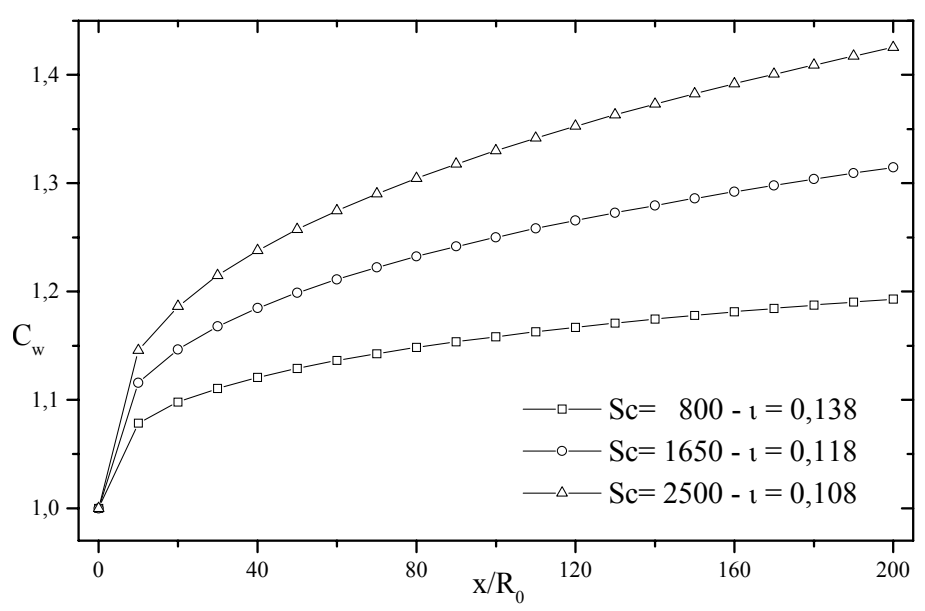

Figura 39. Efeito do número de Schmidt sobre a concentração polarizada em função da posição axial adimensional $\left(\mathrm{x} / \mathrm{R}_{0}\right)$, com $\mathrm{R}_{0}=3,010^{-3} \mathrm{~m}, \mathrm{Re}=700, \mathrm{Re}_{\mathrm{w}}=0,005$. 
Quando aumentamos o número de Schmidt, ocorre o aumento da concentração de polarização, $\mathrm{C}_{\mathrm{w}}$, e dentro do intervalo $800 \leq \mathrm{Sc} \leq 2500$, esta concentração tem variação percentual máxima de aproximadamente de 19\%, o qual pode ser visto na Figura 39. Observando os resultados das Figura 38 e Figura 39 concluímos que o aumento de "Sc" promove um aumento de $19 \%$ na concentração polarizada e uma diminuição $68 \%$ na espessura da camada limite de concentração, e estas tendências estão de acordo com a análise feita na Seção (2.1.4). Pela Equação (103), se aumentarmos "Sc" resultará na diminuição da taxa assintótica, 1 .

Na Figura 40 e na Figura 41 temos as comparações entre os resultados da espessura da camada limite de concentração, provinda da Equação (97), e outros resultados numéricos obtidos por diversos autores.

Para os resultados da espessura da camada limite de concentração dada pela Equação (97), observamos ótimas aproximações entre os resultados numéricos de Song e Elimelech (1995) e Damak e al (2004A), na Figura 40 e na Figura 41, respectivamente. Para determinação da taxa assintótica $\quad \imath=0,158 \quad\left(\mathrm{i}_{\max }=0,262\right) \quad$ e $\quad \imath_{\imath}=0,107 \quad\left(\mathrm{i}_{\max }=0,420\right)$, respectivamente Figura 40 e Figura 41, utilizamos a metodologia da Seção (3.3.2) associada à equação convectiva-difusiva parabólica.

$\mathrm{Na}$ Seção (4.2.4) (Figura 31) tivemos uma boa aproximação, para a espessura da camada limite de concentração, entre o resultado deste trabalho e o resultado obtido por Paris et al. (2002).

Da Figura 34 até a Figura 39 mostramos a coerência da Equação (97) com os fenômenos físicos relacionados à concentração polarizada e à espessura da camada limite de concentração. Conforme mostramos na Figura 31, Figura 40 e Figura 41, os resultados deste 
trabalho para a espessura da camada limite de concentração tem boa aproximação com outros trabalhos da literatura. Desta forma, podemos utilizar a Equação (97), que faz parte da condição de contorno dinâmica, na modelagem matemática do presente trabalho.

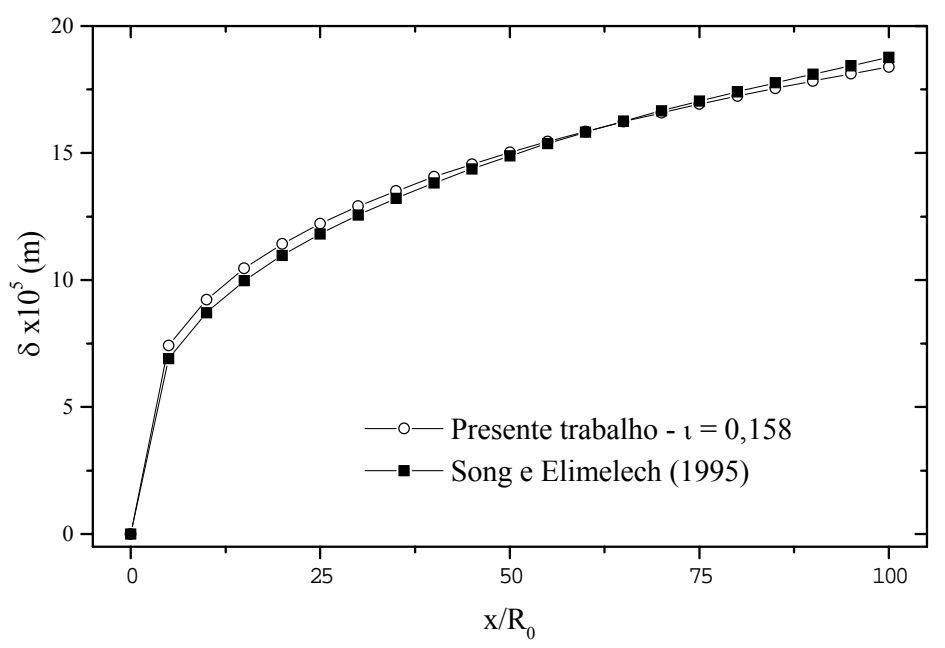

Figura 40. Comparação entre os resultados da espessura da camada limite de concentração deste trabalho com a literatura, com $\mathrm{L}=0,5 \mathrm{~m}, \mathrm{Re}=500, \mathrm{Sc}=1550, \mathrm{Re}_{\mathrm{w}}=0,01 \mathrm{e} \iota_{\max }=0,262$.

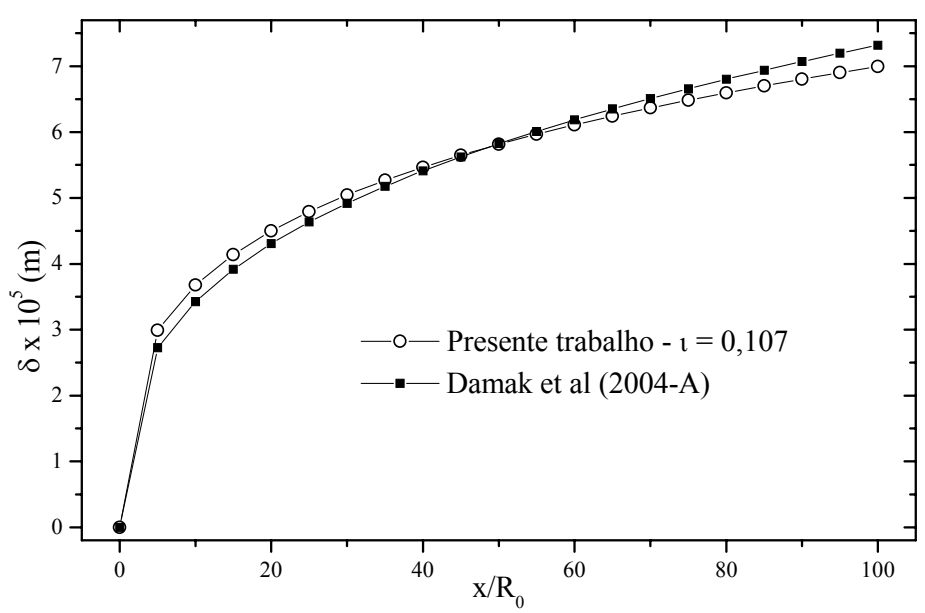

Figura 41. Comparação entre os resultados da espessura da camada limite de concentração deste trabalho (Equação (97)) com a literatura, com $\mathrm{L}=0,3 \mathrm{~m}, \mathrm{Re}=700, \mathrm{Sc}=1100, \mathrm{Re}_{\mathrm{w}}=0,02 \mathrm{e} \iota_{\max }=0,42$. 



\section{Conclusões e Sugestões}

Face ao objetivo principal do trabalho, o escoamento de entrada em regime laminar estacionário e com transferência de massa em tubos permeáveis foi modelado com a equação de conservação das espécies químicas (equação convectiva-difusiva parabólica e elíptica) com o acoplamento de uma condição de contorno na superficie porosa. A técnica híbrida analíticonumérica (GITT) foi aplicada com sucesso na solução da equação de conservação das espécies químicas. As convergências dos resultados das simulações do problema parabólico e elíptico, respectivamente, foram obtidas com 46 e 35 termos.

Desenvolvemos uma expressão matemática original para a espessura da camada limite de concentração que está em função da posição axial e da taxa assintótica, juntamente dos parâmetros adimensionais de Reynolds, Re, Reynolds de permeação, $\mathrm{Re}_{\mathrm{w}}$, e Schmidt, Sc. Os resultados dos perfis das espessuras da camada limite de concentração, $\delta$, em função da posição axial adimensional, $\mathrm{x} / \mathrm{R}_{0}$, estão coerentes com os comportamentos dos mecanismos de transferência de massa na filtração tangencial. As comparações com outros resultados da literatura tiveram boa aproximação, ficando as expressões da espessura da camada limite de concentração e da concentração polarizada habilitadas a serem utilizadas como condição de contorno dinâmico nos processos de filtração tangencial. 
Estabelecemos originalmente uma metodologia para a obtenção da taxa assintótica, a qual ocorre na expressão da camada limite de concentração. Esta taxa assintótica está acoplada à condição de contorno na parede através da concentração polarizada e esta deve ser uma função contínua e diferenciável. Nesta metodologia, o principal critério para a determinação da taxa assintótica é a imposição de aproximação entre a velocidade de permeação, $\mathrm{v}_{\mathrm{w}}$, dada a priori, e o fluxo transmembrana médio, $\mathrm{J}_{\mathrm{m}}$, obtido a partir do modelo do gel. A suposição de que $\mathrm{v}_{\mathrm{W}}=\mathrm{J}_{\mathrm{m}}$ vem da teoria clássica do filme estagnado. $\mathrm{O}$ sucesso desta metodologia foi possível graças à versatilidade da técnica híbrida analítico-numérica GITT, devido ao pequeno tempo de processamento para cada simulação. Observamos que nesta metodologia também podem ser utilizadas técnicas puramente numéricas. Além disso, estabelecemos também uma expressão para a taxa assintótica máxima, que nos dá um critério de coerência nos resultados do perfil de concentração, provindo da simulação via GITT, com o problema físico relacionado à filtração tangencial.

Os resultados para o perfil de concentração e número de Sherwood, para cada um dos modelos: parabólico e elíptico, foram apresentados graficamente para diferentes números de Reynolds, Reynolds de permeação e de Schmidt. Verificaram-se variações e tendências fisicamente coerentes com o problema físico, como por exemplo: com a diminuição do número de Reynolds, a concentração de soluto próxima à superfície permeável aumenta. Observou-se também que o número de Sherwood aumenta com o aumento do número de Reynolds, e com altos números de Reynolds há um menor acúmulo de partículas na superfície permeável da parede do tubo, favorecendo conseqüentemente o processo de transferência de massa.

Com o objetivo especifíco de comparar resultados numéricos provindos da literatura, desenvolvemos a correlação de Gilland-Sherwood. Além disso, tais expressões também 
podem ser utilizadas como ferramenta de comparações, desde que os parâmetros adimensionais estejam dentro dos intervalos estipulados neste trabalho, ou seja, $800 \leq \mathrm{Sc} \leq 2500,200 \leq \operatorname{Re} \leq 700$ e $0,510^{-2} \leq \operatorname{Re}_{\mathrm{w}} \leq 1,410^{-2}$. No trabalho de Damak et al. (2004A) é utilizada como modelo a equação convectiva-difusiva elíptica, a qual é resolvida através da técnica de diferenças finitas, e a correlação de Gilland-Sherwood, Sh, é avaliada a partir do modelo da resistência em série. Na comparação dos resultados produzidos de "Sh" neste trabalho, via GITT, tivemos ótima aproximação com os resultados de Damak et al. (2004A) para o modelo elíptico e uma boa aproximação para o modelo parabólico. Tivemos também boas aproximações em comparações dos resultados da correlação de GillandSherwood desenvolvida neste trabalho com resultados da correlação de Leveque (ZEMAN e ZYDNEY, 1996).

As comparações dos resultados numéricos do fluxo transmembrana local (via GITT) com o resultado numérico da literatura (PARIS et al., 2002), indicam o potencial de modelagem da GITT.

No trabalho de Paris et al. (2002) é utilizada como modelo a equação convectivadifusiva parabólica, onde o fluxo transmembrana local, J, é avaliado a partir do modelo da resistência em série. Na comparação dos resultados de “J”, via GITT, tivemos ótima aproximação para o modelo elíptico e uma boa aproximação para o modelo parabólico. Nesta simulação, pudemos também gerar comparações para a espessura da camada limite de concentração, com boas aproximações, entre o resultado de Paris et al. (2002) e a expressão desenvolvida neste trabalho.

Os resultados via GITT foram afetados por restrições e hipóteses particulares do modelo. No entanto, a metodologia permite determinar o perfil de concentração ao longo do tubo, o coeficiente de transferência de massa $(\mathrm{Sh})$ característico de um processo de filtração 
tangencial.

A análise do efeito do termo difusivo nas equações convectiva-difusiva elíptica e parabólica, mostrou que o termo difusivo axial, $\partial^{2} \mathrm{C} / \partial^{2} \mathrm{X}$, que diferencia o modelo elíptico do parabólico, tem pouca influência nos resultados do perfil de concentração e no adimensional de Sherwood. O comportamento do perfil de concentração sofre influência de no máximo 5\% na saída do tubo, sempre maior no modelo elíptico. Já o comportamento de "Sh" sofre influência de $1 \%$ até $4 \%$ na saída do tubo, contudo o "Sh" ora pode ser maior para o modelo elíptico, ora maior no modelo parabólico, dependendo da combinação dos adimensionais "Re", "Re ${ }_{w} "$ e "Sc". Possivelmente, o comportamento do perfil de concentração e do adimensional de Sherwood pode ter sido afetado pelo fato de termos adotado neste trabalho os perfis de velocidade, U e V, do trabalho de Yuan, Finkelstein e Brooklyn (1956), que descrevem um escoamento em regime laminar completamente desenvolvido.

A correlação de Gilland-Sherwood também foi analisada para os parâmetros: $\operatorname{Re}=700$, $\mathrm{Sc}=800, \mathrm{Re}_{\mathrm{w}}=0.014$ e $\mathrm{Re}=200, \mathrm{Sc}=2500, \mathrm{Re}_{\mathrm{w}}=0.005$, onde o comportamento de "Sh" sofre, respectivamente, influência de 5\% e 9\%, sempre maior no modelo elíptico.

Alguns tópicos abordados neste trabalho poderiam ser estudados em trabalhos futuros. Algumas sugestões para desenvolvimento são:

i. modelar e solucionar, via GITT, os perfis de velocidade do escoamento laminar incompressível, newtoniano e permanente em tubos com superfícies porosas (geometria cilíndrica e retangular), a partir das equações de momentum;

ii. modelar e solucionar, via GITT, os perfis de velocidade do escoamento turbulento em tubos com superfícies porosas (geometria cilíndrica e retangular);

iii. modelar e solucionar, via GITT, os perfis de concentração, a partir das equações 
convectiva-difusiva parabólica e elíptica do escoamento laminar incompressível, newtoniano e permanente em canais com superfícies porosas (geometria retangular). 



\section{Referências Bibliográficas}

AIELLO, G.A., TREFIL, J.S. (1976). The Generalized Entry Flow Problem and the Establishment of Poiseuille Flow in Locally Constricted Tube, Journal Biomechanics, v.9, p.49-54.

APARECIDO, J.B., COTTA, R.M. (1987). Fully Developed Laminar Flow in Trapezoidal Ducts.Anais do IX Congr. Bras. de Eng. Mecânica - IX COBEM, v.1, p.25-28.

APARECIDO, J.B., COTTA, R.M. (1990). Thermally Developing Laminar Flow inside Retangular Ducts, Int. J. Heat Mass Transfer, v.33, n.2, p.341-347.

BACCHIN, P., AIMAR, P., FIELD, R.W. (2006). Critical and Sustainable Fluxes: Theory, Experiments and Applications. Journal of Membrane Science, v.281, p.42-69.

BERMAN, A.S. (1953). Laminar Flow in Channels with Porous Walls, Journal of Applied Physics, v.24, p.1232.

BIXLER, H. J. et al. (1968). The development of a Diafiltration System for Blood Purification, Trans. Am. Soc. Artif. Int. Organs XIV. p.99.

BLATT, W. F. et al. (1970). Solute Polarization and Cake Formation in Membrane Ultrafiltration: Causes, Consequences, and Control Techniques. In Reverse osmosis and 
synthetic membranes, J. E. Flinn, editor, Plenum Press, New York., p. 47.

BOYADJIEV, C.B. (2006). Diffusion Models and Scale-Up, International Journal of Heat and Mass Transfer, v.49, p.796-799.

BOYCE, W. E., DIPRIMA, R. C. (1977). Elementary differential equations and boundary value problems, Wiley, New York.

BRANDY, J.F. (1984). Flow Development in a Porous Channel and Tube, Physics of Fluids, v.27, n.5, p 1061-1067.

BROWN, D.M. et al. (1997). Analysis of Steady Forced Convection in Thermally Developing Turbulent Duct Flows, International Journal of Numerical for Heat \& Fluid Flow, v.7, n.5, p.425-437.

CEBECI, T., BRASHAW, P. (1989). Physical and Computational Aspects of Convective Heat Transfer, Springer-Verlag, New York.

CHERYAN, M. (1998). Ultrafiltration and Microfiltration - Handbook, Technomic Publishing Company, Lancaster, Pennsylvania, p.375.

COTTA, R. M., PIMENTEL, L. C. G. (1998). Developing turbulent duct flow: Hybrid solution via integral transforms and algebric model, International Journal for Numerical Methods for Heat \& Fluid Flow, vol.8, n.1, p.10-26.

COTTA, R.M. (1993). Integral Transforms in Computational Heat and Fluid Flow, CRC Press, Inc., Boca Raton, USA. 
COTTA, R.M. (1994). Benchmark Results in Computational Heat and Fluid Flow: The Integral Transform Method, Int. J. Heat Mass Transfer, v.37, p.381-393.

COTTA, R.M., MIKHAILOV, M.D. (1997). Heat Conduction: Lumped Analysis, Integral Transforms, Symbolic Computation, John Wiley, New York.

CUNHA, F. G. M. (2002). Abordagem Analítico-Numérica da Transferência de Calor e Massa com Efeito da Pressão. Dissertação (Mestrado). UFC, Fortaleza.

DAMAK, K. et al. (2004). Modeling of Crossflow Membrane Separation Processes under Laminar Flow Conditions in tubular Membrane. Desalination, v.168, p.231-239.

DAMAK, K. et al. (2004A). Concentration Polarization in Tubular Membranes - a Numerical Approach, Desalination, v.171, p.139-153.

DERZANSKY, L.J. (1973). An Experimental Study of Reverse Osmosis in a Horizontal Tube: Combined Free and Forced Convection. Ph. D. Thesis. Clarkson College of Technology, Dept. of Chemical Eng.

DENG, X. et al. (1995). Luminal Surface Concentration of Lipoprotein (LDL) and its Effect on the Wall Uptake of Cholesterol by Canine Carotid Arteries, Journal of Vascular Surgey, v. 21, n. 1, p. 135-145.

DINDORE, V.Y., BRILMAN, D.W.F., VERSTEENG, G.F. (2005). Modelling of Cross-Flow Membrane Contactors: Physical Mass Transfer Processes, v. 251, p.209-222.

ELLIOT, R.L., AMIDON, G.L., LIGHTFOOT, E.N. (1980). A Convective Mass Transfer for Determining Intestinal Wall Permeabilities: Laminar Flow in a Circular Tube, J. Theor. Biol, 
v.87, p.757-771.

FARRO, P.C.A. (2003). Ultrafiltração do Soro de Queijo Minas Frescal Pré-Tratado e Microfiltrado: Efeitos da Vazão Volumétrica e da Pressão Transmembrana no Fluxo de Permeado. Dissertação (Mestrado). UNICAMP, Campinas, SP.

FONTES, S.R. (2005). Mass Transfer in Microfiltration with Laminar and Turbulent Flow of Macromolecular Solutions, Journal of Membrane Science, v. 249, p. 207-211.

GERALDES, V. et al. (2001). Flow and Mass Transfer Modeling of Nanofiltration, Journal of Membrane Science, v.191, p. 109-128.

HOEK, E.M.V. et al. (2002). Influence of Crossflow Membrane Filter Geometry and Shear Rate on Colloidal Fouling in Reverse Osmosis and Nanofiltration Separation, Environmental Engineering Science, v.19, n.6, p.337-372.

HUNG, C. I., PERNG, Y.Y. (1991). Flow of Non-Newtonian Fluid in the Entrance of a Tube with Porous Walls, Int. Journal Heat and Fluid Flow, v.12, n.3, p.263-268.

INCROPERA, F.P., De WITT, D.P. (1998). Fundamentos de Transferência de Calor e de Massa, LTC Editora, Rio de Janeiro.

IÓRIO, V. (1989). EDP: Um Curso de Graduação, Coleção Matemática Universitária - SBM, Rio de Janeiro.

IVANTCHEVA, Z.M., COTTA, R.M., MIKHAILOV, M.D. (2004). Symbolic Computational of Correction Function for Polynomial Time Response of Intrinsic Thermocouples, Int. Comm. Heat Mass Transfer, v.31, n. 8, p. 1133-1141. 
IMSL LIBRARY (1991). Math/Lib., Houston, TX.

KIM, A. S., HOEK, E.M.V. (2002). Cake Structure in Dead-End Membrane Filtration: Monte Carlo Simulacions, Environmental Engineering Science, v.19, n.6, p.373-386.

KIM, J.H. et al. (2000). Effect of Membrane Support material on Permeability in the Microfiltration of Brining Wastewater, Desalination, v.140, p.55-65.

KUMAR, V., PANDEY, R.N., UPADHYAY, S.N. (2000). Exact Solution of Convective Mass Transfer Model for Calcium Response of Endothelium, Mathematical Problems in Engineering, v. 5, p.361-377.

LEE, Y., CLARK, M.M. (1998). Modeling of Flux Decline During Crossflow Ultrafiltration of Colloidal Suspensions, Journal of Membrane Science, v.149, p. 181-202.

LEVEQUE, M.D. (1928). Les lois de la transmission de chaleur par convection, Ann. Mines, v.13, p.201.

LUZ NETO, H., QUARESMA, J.N.N., COTTA, R.M. (2002). Natural Convection in ThreeDimentional Porous Cavities: Integral Transform Method, Int. J. Heat and Mass Transfer. v. 45, p. 3013-3032.

MAGNO, R.N.O., MACÊDO, E.N., QUARESMA, J.N.N. (2002). Solutions for the Internal Boundary Layer Equations in Simultaneously Developing Flow of Power-Law Fluids within Parallel Plates Channels, Chemical Engineering Journal, v.87, p. 339-350.

MANSOUR, A.R. (1988). An Exact Solution to a Convective Mass Transfer Laminar Flow Model for Determination of Intestinal Wall Permeabilities, Chemical Engineering Journal, v. 
38, n. 4, p. B29-B32.

MATHEMATICA 5.2 (2005), Copyright (C) 1988-2005 by Wolfram Research, Inc. Mathematica is a registered trademark of Wolfram Research.

MERIN, U., CHERYAN, M. (1980). Factors Affecting Mechanism of Flux Decline During Ultrafiltration of Cottage Cheese Whey, Journal Food Process. Preserv, v.4, p.183.

MICHAELS, A. S. (1965). Kinetics of Water and Salt Transport in Cellulose Acetate Reverse Osmosis Desalination Membranes, J. Colloid. Sci., v.20, p.1034.

MIGNARD, D., GLASS, D.H. (2001). Fouling During the Cross-Flow Ultrafiltration of Protein: a Mass-Transfer Model, v.186, p.133-143.

MIKHAILOV, M.D., ÖZISIK, M.N. (1984). Unifield Analysis and Solutions of Heat and Mass Diffusion, John Wiley, New York.

MS EXCEL 11 (2003). Guia do Usuário, Microsoft Corporation, USA.

NAKAO, S. et al. (1979). Characteristics of Macromolecular Gel Layer Formed on Ultrafiltration tubular Membrane, AIChE J, v.25, p.615.

NOORDMAN, T.R. (2000) .High Flux Ultrafiltration, University of Groningen. Thesis (Doctor). Groningen, Netherlands.

OXARANGO, L. et al. (2004). Laminar Flow in Channels whit Wall Suction or Injection: A New Model to Study Multi-Channel Filtration Systems, Chemical Engineering Science, v.59, p.1039-1051. 
ÖZISIK, M.N. (1968). Boundary Value Problems of Heat Conduction. International Textbook Company, Scranton.

ÖZISIK, M.N. (1980). Heat Conduction. John Wiley, New York.

ÖZISIK, M.N., MURRAY, R.L. (1974). On the Solution of Linear Diffusion Problems with Variable Boundary Conditions, J. Heat Transfer, v.96, p.48-51.

PARIS, J. et al. (2002). Transport Phenomena in Ultrafiltration: a New Two-Dimensional Model Compared with Classical Model, Journal Science Membrane, v.207, p.43-58.

PEREIRA, L.M., PÉREZ-GUERRERO, J.S., COTTA, R.M. (1998). Integral Transform of the Navier-Stokes Equations in Cylindrical Geometry, Computational Mechanics, v.21, p.6070 .

PEREIRA, L.M. (2000). Solução das Equações de Navier-Stokes e da Energia em Coordenadas Cilíndricas Via Transformação Integral. Tese (Doutorado). COPPE Universidade Federal do Rio de Janeiro, Rio de Janeiro.

PÉREZ-GUERRERO, J.S., COTTA, R.M. (1992). Integral Transform Methods for NavierStokes Equations in Stream Function-Only Formulation, Int. J. Num. Meth. Fluids, v.15, p.399-409.

PÉREZ-GUERRERO, J.S., COTTA, R.M. SCOFANO NETO, F. (1993). Integral Transform Methods for Navier-Stokes Equations for Incompressible Laminar Flow in Channels. Proc. $8^{\text {th }}$. Inc. Conf. on Numerical Methods in Laminar and Turbulent Flow, Swansca, Inglaterra, v.2, p.1195-1206. 
PÉREZ-GUERRERO, J.S., COTTA, R.M. (1995). Integral Transform Solution of Developing Laminar duct flow in Navier-Stokes Formulation, Int. J. Num. Meth. Fluids, v.20, p.12031213.

PÉREZ-GUERRERO, J.S., COTTA, R.M. (1996). Benchmark Integral Transform Results for Flow over a Backward-Facing Step, Computers \& Fluids, v.25, p.527-540.

PORTER, M. C. (1972). Concentration Polarization with Membrane Ultrafiltration, I\&EC Prod. Res., v.11(3), p.234.

QUAILE, J.P., LEVY, E.K. (1975). Laminar Flow in a Porous Tube with Suction, Journal of Heat Tranfer, v.2, p.66-71.

QUEIROZ, V.M.S. (2004). Estudo Experimental do Escoamento e da Concentração de Mistura no Processo de Filtração Tangencial de Suspensões Macromoleculares. Dissertação (Mestrado). EESC, USP, São Carlos.

RICHARDSON, C.J., NASSEHI, V. (2003) Finite element modelling of concentration profles in flow omains with curved porous boundaries. Chemical Engineering Science, v.58, p.24912503

RIPPERGER, S., ALTMANN, J. (2002). Crossflow Microfiltration - State of the Art, Separation and Purification Technology, v.26, p.19-31.

ROMERO, C.A., DAVIS, R.H. (1988). Global Model of Crossflow Microfiltration Based on Hydrodynamic Particle Diffusion, Journal of Membrane Science, v.39, p.157-185.

SCIRUS, for information scientific (2007). Disponível em: http://www.scirus.com/srsapp/. 
Acesso em 12.nov.2007.

SCOFANO NETO, F. et al. (1989). Alternative Analytical Approach to Diffusion Problems with General Coupling at the Boundaries”, Anais do X Congr. Bras. Eng. Mecânica, p.173176, Rio de Janeiro.

SILVA, E.F., PÉREZ-GUERRERO, J.S., COTTA, R.M. (1999). Integral Transform Solution of Boundary Layer Equation in Stream Function-Only Formulation, International Journal of Non-Linear Mechanics, v.34, p.51-61.

SILVA, J.B.C., COTTA, R.M., APARECIDO, J.B. (1992). Analytical Solutions to Simultaneously Developing Laminar Flow Inside Parallel-Plate Channel, Int. J Heat Mass Transfer, v.35, n.4, p.887-895.

SILVA, J.M. (2008). Modelagem Numérica do Escoamento num Tubo Permeável Aplicada ao Processo de Filtração Tangencial. Tese (Doutorado). EESC-USP, São Carlos.

SONG, L., ELIMELECH, M. (1995). Theory of Concentration Polarization in Crossflow Filtration, Journal Chem. Soc. Faraday Trans., v.91, p.3389-3398.

SPARROW, E. M., LIN S. J., LUNDGREN, T. S. (1964). Flow development in the hydrodynamics entrance region of tubes and ducts, Journal of Physics Fluids, v.7, p.338-347.

STREETER, V. L. (1979). Mecânica dos Fluidos, McGraw-Hill, São Paulo.

RANJAN, R. et al. (2004). Mass Transfer coefficient with Suction for Laminar NonNewtonian Flow in Application to Membrane Separations, Journal of Food Engineering, v.64, p.53-61. 
TERRIL, R.M. (1964). Laminar Flow in a uniformly porous channel, Aeronaut, v.15, p.297.

TERRIL, R. M. (1983). Laminar flow in a Porous Tube, Journal of Fluids Engineering, v.105, p.303.

TRETTIN, D. R. (1980). An Investigation of Mass Transfer Mechanisms in Ultrafiltration. Thesis (Doctor). Lawrence University, Appleton, USA.

TRUSKEY, G.A. et al. (2004). Transport Phenomena in Biological Systems, Prentice Hall.

UNITED STATES ENVIRONMENTAL PROTECTION AGENCY (2003). Membrane Filtration Guidance Manual. Disponível em http://permanent.access.gpo.gov/websites/epagov/www.epa.gov/safewater/lt2/pdfs/guide_lt2_ membranefiltration_draft.pdf. Acesso em 10.nov. 2007.

VENEZUELA, A.L., FONTES, S.R. (2007). Analytical-Numerical Modeling of Convective Laminar Flow in a Permeable Tube Associated with the Cross-Flow Process - AxialConvective-Difusive Equation, Proceedings DINCON 2007, $6^{\text {th }}$ Brazilian Conference on Dynamics, Control and Their, São José do Rio Preto, Brasil.

VENEZUELA, A.L., PÉREZ-GUERRERO, J.S., FONTES, S.R. (2008). Hybrid Modeling of Convective Laminar Flow in a Permeable Tube Associated with the Cross-Flow. Communications in Nonlinear Science and Numerical Simulation, Doi:10.1016/j.cnsns.2007.11.009. (in press)

WANG, G., DENG, X., GUIDOIN, R. (2003). Concentration Polarization of Macromolecules in Canine Carotid Arteries and its Implication for the Localization of Atherogenesis, Jourmal of Biomechanics, v.36, p.45-51. 
WEISBERG, S. (2005). Applied Linear Regression, John Weley \& Suns, inc., Publications, New Jersey.

WEISSBERG, H. (1959). Laminar Flow in the Entrance Region of a Porous Pipe, Physics of Fluids, v.2, p.510.

WELTY, J.R., WICKS C.E., WILSON R.E. (1969). Fundamentals of Momentum, Heat and Mass Transfer, John Wiley, New York.

WIJMANS, J.G. et al. (1985). Hydrodynamic Resistance of Concentration Polarization Boundary Layers in Ultrafiltration, Journal Membrane Science, v.22, p.117.

WU, D. et al. (1991). A New Meted for Modeling the Time-Dependence of Permeation Flux in Ultrafiltration, Food and Bioproducts Processing, v.69 (C2), p.77-82

YEH, H.M. et al. (2004). Momentum Balance Analysis of Flux and Pressure Declines in Membrane Ultrafiltration along Tubular Modules, Journal of Membrane Science, v.241, p.335-345.

YUAN, S.W., FUNKELSTEIN, A.B., BOOKLYN, N.Y. (1956). Laminar Pipe Flow with Injection and Suction through a Porous Wall, Translation of ASME, v.78, p.719-724.

ZEMAN, L.J., ZYDNEY, A.L. (1996). Microfiltration and Ultrafiltration - Principles and Applications, Marcel Dekker, New York.

ZYDNEY, A.L. (1997) Stagnant Film Model for Concentration Polarization in Membrane Systems, Journal of Membrane Science, v.130, p.275-281. 

Apêndices

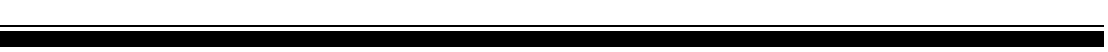





\section{APÊNDICE 1: Perfil de CONCENTRAÇÃo PARA UM ESCOAMENTO LAMinar COMPLETAMENTE DESENVOLVIDO.}

Consideremos um escoamento completamente desenvolvido, cuja concentração é representada pelo potencial $\mathrm{F} \equiv \mathrm{F}(\mathrm{R} ; \mathrm{X})$, em regime estacionário, onde não ocorrem reações químicas e a difusividade é constante. Pelo fato do regime do escoamento ser completamente desenvolvido, a difusão do soluto passa a ser unidimensional e na direção radial. Pela lei de conservação mássica, temos que a equação de difusão, em coordenadas cilíndricas, é dada na forma adimensional por (INCROPERA, 1998):

$$
\frac{1}{P e R} \frac{\partial}{\partial R}\left(R \frac{\partial F}{\partial R}\right)=0, \quad 0<R \leq 1
$$

com as condições de contorno $\mathrm{F}(0 ; \mathrm{X})=1$ e $\mathrm{F}(1 ; \mathrm{X})=\mathrm{C}_{\mathrm{w}}(\mathrm{X})$.

Na Equação (108) aplicamos a integração e temos o seguinte:

$$
\int \frac{\partial}{\partial \mathrm{R}}\left(\mathrm{R} \frac{\partial \mathrm{F}(\mathrm{R} ; \mathrm{X})}{\partial \mathrm{R}}\right) \mathrm{dR}=0 \Rightarrow \frac{\partial \mathrm{F}(\mathrm{R} ; \mathrm{X})}{\partial \mathrm{R}}=\frac{\mathrm{F}_{1}}{\mathrm{R}}
$$

e daí obtemos:

$$
\mathrm{F}(\mathrm{R} ; \mathrm{X})=\mathrm{F}_{1} \ln (\mathrm{R})+\mathrm{F}_{2}, \quad 0<\mathrm{R} \leq 1
$$

sendo $F_{1}$ e $F_{2}$ constantes de integração com relação ao eixo radial. 
$\mathrm{Na}$ Equação (109) o potencial " $\mathrm{F}$ ” não está definido em $\mathrm{R}=0$, daí utilizaremos as seguintes condições de contorno: $\mathrm{F}\left(\mathrm{R}_{\min } ; \mathrm{X}\right)=1$ e $\mathrm{F}(1 ; \mathrm{X})=\mathrm{C}_{\mathrm{w}}(\mathrm{X})$, sendo $\mathrm{R}_{\min }$ definido de acordo com a Equação (123) do Apêndice (2).

Utilizando as condições de contorno acima, determinamos uma expressão matemática para o perfil de concentração " $F$ " relacionada a um escoamento completamente desenvolvido:

$$
\mathrm{F}(\mathrm{R} ; \mathrm{X})=\left(\frac{1-\mathrm{C}_{\mathrm{w}}(\mathrm{X})}{\ln \left(\mathrm{R}_{\text {min }}\right)}\right) \ln (\mathrm{R})+\mathrm{C}_{\mathrm{w}}(\mathrm{X}), \quad \mathrm{R}_{\text {min }}<\mathrm{R} \leq 1
$$




\section{Apêndice 2: Problema AUXiliar}

Os problemas originais são equações diferenciais parciais, juntamente com as condições de contorno, condição de entrada e de saída, que modelam determinado processo físico. A GITT (COTTA, 1993) destaca que os problemas auxiliares provindos de problemas originais, e que envolvem dificuldades computacionais, devem ser evitados e assim considerar problemas auxiliares mais tratáveis e que ainda contenham informações sobre o problema original.

No desenvolvimento da GITT, Cotta (1993) estabelece um problema auxiliar generalizado para uma equação diferencial parcial parabólica também generalizada, utilizando a CITT. Seguiremos seus passos e considerando como problema original a equação axial convectiva-difusiva parabólica (Equação (20)) que modela certos problemas associados aos processos de filtração tangencial, conforme vimos na Seção (2.1.6). Na forma adimensional, a equação axial convectiva-difusiva parabólica é dada por:

$$
\mathrm{U}(\mathrm{X}, \mathrm{R}) \frac{\partial \mathrm{C}(\mathrm{X}, \mathrm{R})}{\partial \mathrm{X}}=\frac{1}{\mathrm{Pe} \mathrm{R}} \frac{\partial}{\partial \mathrm{R}}\left(\mathrm{R} \frac{\partial \mathrm{C}(\mathrm{X}, \mathrm{R})}{\partial \mathrm{R}}\right)
$$

sendo, $0 \leq \mathrm{R} \leq 1,0 \leq \mathrm{X} \leq \xi, \xi=\mathrm{L} / \mathrm{R}_{0}$, com as seguintes condições:

- no centro do tubo, $R=0,0 \leq X \leq \xi$ :

$$
\mathrm{C}(\mathrm{X}, 0)=1
$$


- na parede do tubo, $\mathrm{R}=1,0 \leq \mathrm{X} \leq \xi$ :

$$
\mathrm{C}(\mathrm{X}, 1)=\mathrm{C}_{\mathrm{w}}(\mathrm{X})
$$

- na entrada do tubo, $\mathrm{X}=0,0 \leq \mathrm{R} \leq 1$ :

$$
\mathrm{C}(0, \mathrm{R})=1
$$

A equação diferencial parcial parabólica, Equação (111), tem condição de contorno não homogêneos, Equações (112) e (113). Com o objetivo de se obter uma solução aproximada pelo método de separação de variáveis (ou método de Fourier), Cotta (1993) sugere tomar-se uma versão apropriada do problema original, Equação (111), com condições de contorno homogênea. Esta homogeneização é possível de ser feita com a utilização do filtro, $\mathrm{C}(\mathrm{X}, \mathrm{R})=\mathrm{C}_{\mathrm{H}}(\mathrm{X}, \mathrm{R})+\mathrm{F}(\mathrm{R} ; \mathrm{X})$. Assim podemos tomar uma apropriada equação diferencial parcial parabólica com condição de contorno homogênea:

$$
\mathrm{U}_{2}(\mathrm{X}) \frac{\partial \mathrm{C}_{\mathrm{H}}(\mathrm{X}, \mathrm{R})}{\partial \mathrm{X}}=\frac{1}{\operatorname{Pe} \mathrm{R}} \frac{\partial}{\partial \mathrm{R}}\left(\mathrm{R} \frac{\partial \mathrm{C}_{\mathrm{H}}(\mathrm{X}, \mathrm{R})}{\partial \mathrm{R}}\right)
$$

sendo $0 \leq \mathrm{X} \leq \xi, 0 \leq \mathrm{R} \leq 1$ e as condições de contorno homogêneas:

$$
\begin{aligned}
& C_{H}(X, 0)=0 \\
& C_{H}(X, 1)=0
\end{aligned}
$$

e a condição inicial: $C_{H}(0, R)=0$.

Seguindo o método de Fourier, consideremos que o potencial de concentração homogêneo possa ser escrito como:

$$
\mathrm{C}_{\mathrm{H}}(\mathrm{X}, \mathrm{R})=\Gamma(\mathrm{X}) \psi(\mathrm{R})
$$

Substituindo a Equação (118), nas Equações (115), (116) e (117) e considerando: $\Gamma(\mathrm{X}) \neq 0,0 \leq \mathrm{X} \leq \xi$, obtemos: 


$$
\frac{\mathrm{d} \Gamma(\mathrm{X})}{\mathrm{dX}}+\frac{1}{\mathrm{Pe}_{2}(\mathrm{X})} \beta^{2} \Gamma(\mathrm{X})=0
$$

e

$$
\frac{1}{\mathrm{R}} \frac{\mathrm{d}}{\mathrm{dR}}\left(\mathrm{R} \frac{\mathrm{d} \psi(\mathrm{R})}{\mathrm{dR}}\right)+\beta^{2} \psi(\mathrm{R})=0
$$

sendo $0 \leq \mathrm{R} \leq 1$, as condições de contorno:

$$
\begin{aligned}
& \psi(0)=0 \\
& \psi(1)=0
\end{aligned}
$$

As Equações (120), (121) e (122) caracterizam o problema auxiliar, ou problema de autovalor, o qual é um problema do tipo Sturm-Liouville. A Equação (120) é a conhecida equação diferencial de Bessel de ordem zero, com $\beta$ e $\psi$ sendo, respectivamente, os autovalores e as autofunções.

Özisik (1980) estabelece o fato de que, para regiões finitas, os autovalores, $\beta_{\mathrm{i}}$, $i=1,2,3, \ldots$, formam um espectro discreto. Portanto, para cada valor possível do autovalor, $\beta_{i}$, estará associada à solução do problema auxiliar, Equações (120), ou seja, existirá uma autofunção $\psi\left(\beta_{\mathrm{i}}, \mathrm{R}\right)=\psi_{\mathrm{i}}(\mathrm{R}), \mathrm{i}=1,2,3, \ldots$, que permite determinar a solução completa para $\mathrm{C}_{\mathrm{H}}(\mathrm{X}, \mathrm{R})$, a qual deve ser construída como combinação linear de todas as possíveis soluções auxiliares, $\psi_{\mathrm{i}}(\mathrm{R})$.

Nos casos dos problemas originais serem equações diferenciais parciais parabólicas ou elípticas, com condições de contorno não homogêneas, o procedimento é o mesmo que o realizado neste apêndice. Para problemas originais em coordenadas cilíndricas, Cotta (1993) toma o mesmo problema auxiliar, isto é, a equação de Bessel de ordem zero (Equações (120)).

No trabalho de Özisik (1980) estão tabeladas as formulações dos autovetores e das 
autofunções relacionados ao problema auxiliar em coordenadas cilíndricas para a região entre dois tubos concêntricos de raio $\mathrm{R}=\mathrm{R}_{\min }$ e $\mathrm{R}=1, \mathrm{R}_{\min } \leq \mathrm{R} \leq 1$ (Figura 42), cujas condições de contorno para o problema de autovalor são dadas na posição radial $\mathrm{R}=\mathrm{R}_{\min } \mathrm{e} \mathrm{R}=1$.

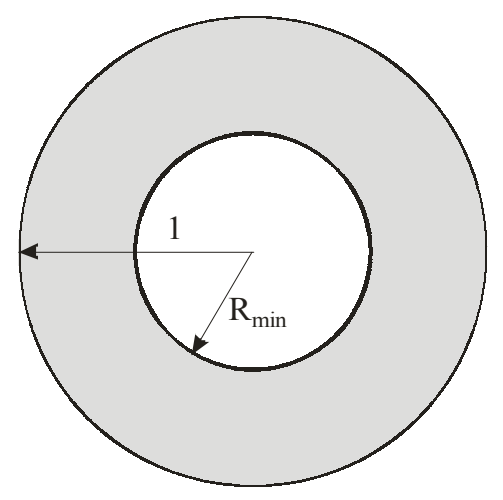

Figura 42. Representação da região entre dois tubos concêntricos.

No problema dado pela Equação (120), temos as condições de contorno no eixo radial, $(\mathrm{R}=0$, Equação (121)), e na parede do tubo, $(\mathrm{R}=1$, Equação (122)). Se considerarmos $\mathrm{R}_{\min } \ll 1$, então teremos a compatibilidade entre as condições de contorno dadas pelas Equações (121) e (122), com as condições de contorno do problema auxiliar da região entre os tubos concêntricos expressas por Özisik (1980). Desta forma, como sugere a GITT em tomar um problema auxiliar tratável e contendo informações do problema original, o problema auxiliar dado pelas Equações (120), (121) e (122), será escrito como:

$$
\frac{1}{\mathrm{R}} \frac{\mathrm{d}}{\mathrm{dR}}\left(\mathrm{R} \frac{\mathrm{d} \psi_{\mathrm{i}}(\mathrm{R})}{\mathrm{dR}}\right)+\beta_{\mathrm{i}}^{2} \psi_{\mathrm{i}}(\mathrm{R})=0, \quad \mathrm{i}=1,2,3, \ldots
$$

sendo $\mathrm{R}_{\min } \leq \mathrm{R} \leq 1$ e as condições de contorno:

$$
\begin{aligned}
& \psi_{\mathrm{i}}\left(\mathrm{R}_{\text {min }}\right)=0 \\
& \psi_{\mathrm{i}}(1)=0
\end{aligned}
$$

Substituindo $\mathrm{U}_{2}(\mathrm{X})$ por $\mathrm{U}(\mathrm{X}, \mathrm{R})=\mathrm{U}_{1}(\mathrm{R}) \mathrm{U}_{2}(\mathrm{X})$ na Equação (115) e procedendo analogamente ao que desenvolvemos anteriormente, obtemos a equação de Graetz, dada por: 


$$
\frac{1}{\mathrm{R}} \frac{\mathrm{d}}{\mathrm{dR}}\left(\mathrm{R} \frac{\mathrm{d} \psi(\mathrm{R})}{\mathrm{dR}}\right)+\beta^{2} \mathrm{U}_{1}(\mathrm{R}) \psi(\mathrm{R})=0
$$

sendo $0 \leq \mathrm{R} \leq 1$, e as condições de contorno:

$$
\begin{aligned}
& \psi(0)=0 \\
& \psi(1)=0
\end{aligned}
$$





\section{APÊNDICE 3: O PAR TRANSFORMADA - INVERSA}

No Apêndice (2) temos o problema auxiliar, Equações (123), (124) e (125), relacionado aos problemas originais envolvidos na filtração tangencial, onde $\psi_{i}, i=1,2,3, \ldots$, são as autofunções (conhecidas) associadas aos autovalores $\beta_{\mathrm{i}}$. Iório (1989) mostra que tais autofunções são continuas.

Seja o espaço de todas as funções continuas, $C_{\mathbb{R}}\left(\left[R_{\min }, 1\right]\right)$, isto é, se $\psi_{\mathrm{i}} \in \mathrm{C}_{\mathbb{R}}\left(\left[\mathrm{R}_{\min }, 1\right]\right), \operatorname{logo} \psi_{\mathrm{i}}:\left[\mathrm{R}_{\min }, 1\right] \rightarrow \mathbb{R}$ é continua (IÓRIO, 1989). Pela Equação (123) do Apêndice (2), definimos o intervalo $\mathrm{R}_{\min } \leq \mathrm{R} \leq 1$, sendo $\mathrm{R}_{\min } \ll 1$. Definimos um produto interno em $\mathrm{C}_{\mathbb{R}}\left(\left[\mathrm{R}_{\text {min }}, 1\right]\right)$ como sendo:

$$
\left\langle\psi_{\mathrm{i}}(\mathrm{R}), \psi_{\mathrm{j}}(\mathrm{R})\right\rangle=\int_{\mathrm{R}_{\min }}^{1} \mathrm{R} \psi_{\mathrm{i}}(\mathrm{R}) \psi_{\mathrm{j}}(\mathrm{R}) \mathrm{dR}
$$

onde $\psi_{\mathrm{i}}$ e $\psi_{\mathrm{j}}, \mathrm{i}, \mathrm{j}=1,2,3, \ldots$, são autofunções associadas a autovalores distintos $\beta_{\mathrm{i}}$ e $\beta_{\mathrm{j}}, \mathrm{i} \neq \mathrm{j}$. Logo $\psi_{\mathrm{i}}$ e $\psi_{\mathrm{j}}$ são ortogonais com relação ao produto interno dado pela Equação (129), isto é, $\left\langle\psi_{\mathrm{i}}(\mathrm{R}), \psi_{\mathrm{j}}(\mathrm{R})\right\rangle=0$, se $\mathrm{i} \neq \mathrm{j}$ (IÓRIO, 1989 e COTTA, 1993). Assim temos:

$$
\left\langle\psi_{\mathrm{i}}(\mathrm{R}), \psi_{\mathrm{j}}(\mathrm{R})\right\rangle=\int_{\mathrm{R}_{\min }}^{1} \mathrm{R} \psi_{\mathrm{i}}(\mathrm{R}) \psi_{\mathrm{j}}(\mathrm{R}) \mathrm{dR}=\left\{\begin{array}{cc}
0, & \text { se } \mathrm{i} \neq \mathrm{j} \\
\mathrm{N}_{\mathrm{i}}, & \text { se } \mathrm{i}=\mathrm{j}
\end{array}\right.
$$


Quando $i=j$, temos $N_{i}=\int_{R_{\min }}^{1} \psi_{i}^{2}(R) d R$, a qual é chamada de integral de ortonormalização. A norma é dada por:

$$
\left\|\psi_{\mathrm{i}}\right\|=\sqrt{\left\langle\psi_{\mathrm{i}}, \psi_{\mathrm{i}}\right\rangle}=\mathrm{N}_{\mathrm{i}}^{1 / 2}
$$

As autofunções normalizadas são dadas por:

$$
\widetilde{\psi}_{\mathrm{i}}(\mathrm{R})=\frac{\psi_{\mathrm{i}}(\mathrm{R})}{\mathrm{N}_{\mathrm{i}}^{1 / 2}}
$$

Pelo principio da superposição (COTTA, 1993 e IÓRIO, 1989) a GITT supõe que todo potencial homogêneo $\mathrm{C}_{\mathrm{H}}(\mathrm{X}, \mathrm{R})$ possa ser construído por uma expansão de autofunções (COTTA, 1993), ou seja:

$$
\mathrm{C}_{\mathrm{H}}(\mathrm{X}, \mathrm{R})=\sum_{\mathrm{i}=1}^{\infty} \overline{\mathrm{A}}_{\mathrm{i}}(\mathrm{X}) \psi_{\mathrm{i}}(\mathrm{R})
$$

onde as autofunções $\psi_{\mathrm{i}}(\mathrm{R})$ são obtidas do problema auxiliar (Apêndice (2)).

Para obter uma expressão geral dos coeficientes $\overline{\mathrm{A}}_{\mathrm{i}}, \mathrm{i}=1,2,3, \ldots$, aplicaremos $\mathrm{o}$ operador $\int_{R_{\min }}^{1} R \psi_{i}(R) d R$ sobre a Equação (133), assim:

$$
\int_{\mathrm{R}_{\min }}^{1} \mathrm{R} \psi_{\mathrm{i}}(\mathrm{R}) \mathrm{C}_{\mathrm{H}}(\mathrm{X}, \mathrm{R}) \mathrm{dR}=\int_{\mathrm{R}_{\min }}^{1} \mathrm{R} \psi_{\mathrm{i}}(\mathrm{R})\left(\sum_{\mathrm{j}=1}^{\infty} \overline{\mathrm{A}}_{\mathrm{j}}(\mathrm{X}) \psi_{\mathrm{j}}(\mathrm{R})\right) \mathrm{dR}
$$

No segundo membro da equação acima, aplicamos as propriedades do cálculo integral e obtemos:

$$
\int_{\mathrm{R}_{\min }}^{1} \mathrm{R} \psi_{\mathrm{i}}(\mathrm{R}) \mathrm{C}_{\mathrm{H}}(\mathrm{X}, \mathrm{R}) \mathrm{dR}=\sum_{\mathrm{j}=1}^{\infty} \overline{\mathrm{A}}_{\mathrm{j}}(\mathrm{X}) \int_{\mathrm{R}_{\min }}^{1}\left(\mathrm{R} \psi_{\mathrm{i}}(\mathrm{R}) \psi_{\mathrm{j}}(\mathrm{R})\right) \mathrm{dR}
$$

Definindo: 


$$
\bar{C}_{i}(X)=\int_{R_{\min }}^{1} R \psi_{i}(R) C_{H}(X, R) d R
$$

e substituindo a Equação (135) na Equação (134) e também utilizando o conceito da Equação (130), obtemos $\overline{\mathrm{C}}_{\mathrm{i}}(\mathrm{X})=\overline{\mathrm{A}}_{\mathrm{i}} \mathrm{N}_{\mathrm{i}}$. Daí:

$$
\overline{\mathrm{A}}_{\mathrm{i}}=\frac{1}{\mathrm{~N}_{\mathrm{i}}} \overline{\mathrm{C}}_{\mathrm{i}}(\mathrm{X}), \quad \mathrm{i},=1,2,3, \ldots
$$

Substituindo a Equação (136) na Equação (133), obtemos:

$$
\mathrm{C}_{\mathrm{H}}(\mathrm{X}, \mathrm{R})=\sum_{\mathrm{i}=1}^{\infty} \frac{1}{\mathrm{~N}_{\mathrm{i}}} \overline{\mathrm{C}}_{\mathrm{i}}(\mathrm{X}) \psi_{\mathrm{i}}(\mathrm{R})
$$

Utilizando as autofunções normalizadas, Equação (132), nas Equações (135) e (137), obtemos o seguinte par transformada-inversa:

$$
\begin{array}{ll}
\bar{C}_{i}(X)=\int_{R_{\min }}^{1} \mathrm{R} \widetilde{\psi}_{\mathrm{i}}(\mathrm{R}) \mathrm{C}_{\mathrm{H}}(\mathrm{X}, \mathrm{R}) \mathrm{dR} & \text { - Transformada } \\
\mathrm{C}_{\mathrm{H}}(\mathrm{X}, \mathrm{R})=\sum_{\mathrm{i}=1}^{\infty} \overline{\mathrm{C}}_{\mathrm{i}}(\mathrm{X}) \tilde{\psi}_{\mathrm{i}}(\mathrm{R}) & \text { - Inversa }
\end{array}
$$





\section{Apêndice 4: AvaliaÇão dos Parâmetros da CorrelaÇão de Gilland- SHERWOOD.}

Na Equação (46) temos a correlação de Gilland-Sherwood, a qual pode ser escrita como (DAMAK, et al., 2004A):

$$
\operatorname{Sh}=s\left(\operatorname{ReSc} \frac{1}{X}\right)^{\alpha}\left(1+w \operatorname{Re}^{\zeta} \operatorname{Sc}^{\gamma} \operatorname{Re}_{w}^{\lambda}\right)
$$

$\operatorname{com} \mathrm{X}, \mathrm{s}, \mathrm{w}, \alpha, \zeta, \gamma$ e $\lambda$ sendo, respectivamente, o eixo axial adimensional e os parâmetros que devemos estimar através da Regressão Linear Simples e Múltipla.

Com o objetivo de tornar linear a Equação (140), e a utilização dos resultados do número de Sherwood, $\mathrm{Sh}_{\mathrm{mod}}$, provindos da solução da modelagem, via GITT, vamos reescrevê-la como segue:

$$
\frac{\mathrm{Sh}_{\text {mod }}}{\mathrm{Sh}_{\text {aprox }}}-1=\mathrm{w} \operatorname{Re}^{\zeta} \mathrm{Sc}^{\gamma} \operatorname{Re}_{\mathrm{w}}^{\lambda}
$$

com:

$$
\mathrm{Sh}_{\mathrm{aprox}}=\mathrm{s}(\operatorname{ReSc} / \mathrm{X})^{\alpha}
$$

Na Equação (141) e Equação (142), aplicamos o logaritmo natural, ln:

$$
\ln \left(\frac{\mathrm{Sh}_{\mathrm{mod}}}{\mathrm{Sh}_{\text {aprox }}}-1\right)=\ln (\mathrm{w})+\zeta \ln (\mathrm{Re})+\gamma \ln (\mathrm{Sc})+\lambda \ln \left(\mathrm{Re}_{\mathrm{w}}\right)
$$




$$
\ln \left(\mathrm{Sh}_{\text {aprox }}\right)=\ln (\mathrm{s})+\alpha \ln (\operatorname{ReSc} / \mathrm{X})
$$

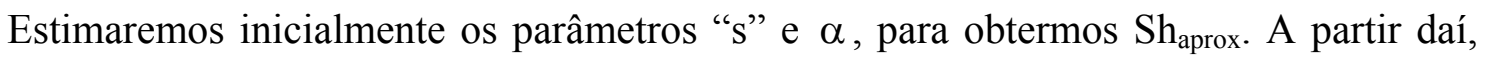
avaliaremos $\mathrm{w}, \zeta, \gamma$ e $\lambda$, para determinarmos a correlação final, Sh.

Utilizaremos a formatação do "resumo de dados" fornecido pelo MS Excel 11 (2003), que consta da "ferramenta: análise de dados" na macro "Regressão".

Nos itens (1) e (2) abaixo, calcularemos os parâmetros das correlações de GillandSherwood a partir dos resultados, obtidos via GITT, dos problemas convectivo-difusivo parabólico e elíptico. A análise estatística pode ser encontrada no trabalho de Weisberg (2005).

(1) Correlação de Gilland-Sherwood a partir do problema convectivo-difusivo parabólico.

A Tabela 9 será construída a partir da Equação (144), na qual X1 e Y1 são as variáveis linearizadas, respectivamente, independente e dependente.

Tabela 9 . Valores de "Sh" provindos da solução do modelo em referência, via GITT, para determinação de $\mathrm{Sh}_{\text {aprox }}$, com $\mathrm{R}_{0}=3.010^{-3} \mathrm{~m}, 200 \leq \mathrm{Re} \leq 800,800 \leq \mathrm{Sc} \leq 2500, \mathrm{Re}_{\mathrm{w}}=0,005$ e $\varepsilon=0,01$.

\begin{tabular}{|c|c|c|c|c|c|c|}
\hline $\mathrm{Sh}_{\text {mod }}$ & $\mathrm{X}$ & $\mathrm{Re}$ & $\mathrm{Sc}$ & $\mathrm{Re} \mathrm{Sc} / \mathrm{X}$ & $\ln (\mathrm{Sh})$ & $\ln (\mathrm{Re} \mathrm{Sc} / \mathrm{X})$ \\
\cline { 5 - 7 } & & & & & $\mathrm{Y} 1$ & $\mathrm{X} 1$ \\
\hline 26,91855 & 10 & 200 & 800 & 16000,00 & 3,2928 & 9,6803 \\
\hline 10,72091 & 100 & 200 & 800 & 1600,00 & 2,3722 & 7,3778 \\
\hline 42,72997 & 10 & 200 & 2500 & 50000,00 & 3,7549 & 10,8198 \\
\hline 19,73797 & 100 & 200 & 2500 & 5000,00 & 2,9825 & 8,5172 \\
\hline 41,32595 & 10 & 700 & 800 & 56000,00 & 3,7215 & 10,9331 \\
\hline 15,52603 & 100 & 700 & 800 & 5600,00 & 2,7425 & 8,6305 \\
\hline 58,99228 & 10 & 700 & 2500 & 175000,00 & 4,0774 & 12,0725 \\
\hline 26,32951 & 100 & 700 & 2500 & 17500,00 & 3,2707 & 9,7700 \\
\hline
\end{tabular}


A Tabela 10 contém as informações necessárias para análise estatística dos parâmetros “s” e $\alpha$, com $\widetilde{R}, \tilde{\mathrm{F}}$ e $\tilde{\mathrm{t}}$ sendo, respectivamente, o coeficiente de determinação, a distribuição F de probabilidade e a distribuição de probabilidade $t$ de Student.

Tabela 10. Quadro para análise estatística dos parâmetros "s" e $\alpha$.

\begin{tabular}{|c|c|c|c|c|}
\hline \multicolumn{5}{|c|}{ Estatística de regressão } \\
\hline \multicolumn{3}{|l|}{$\widetilde{R}^{2}$} & \multicolumn{2}{|c|}{0,9766} \\
\hline \multicolumn{3}{|c|}{$\widetilde{\mathrm{R}}^{2}$ ajustado } & \multicolumn{2}{|c|}{0,9727} \\
\hline \multicolumn{3}{|c|}{ Erro padrão } & \multicolumn{2}{|c|}{0,0938} \\
\hline \multicolumn{3}{|c|}{ Observações } & \multicolumn{2}{|r|}{8} \\
\hline \multicolumn{3}{|c|}{$\tilde{\mathrm{F}}$ (Calculado) } & \multicolumn{2}{|c|}{250,8238} \\
\hline \multirow{2}{*}{\multicolumn{3}{|c|}{$\begin{array}{l}\tilde{F} \text { (Tabelado - confiança: 95\%) } \\
\tilde{\mathrm{t}} \text { (Tabelado - confiança: 95\%) }\end{array}$}} & \multicolumn{2}{|c|}{5,9873} \\
\hline & & & \multicolumn{2}{|c|}{2,4469} \\
\hline \multicolumn{2}{|r|}{ Coeficientes } & \multicolumn{2}{|c|}{ Erro padrão } & $\tilde{\mathrm{t}}$ (Calculado) \\
\hline Interseção & $-0,2959$ & \multicolumn{2}{|c|}{0,2280} & $-1,2979$ \\
\hline $\mathrm{X} 1$ & 0,3674 & \multicolumn{2}{|c|}{0,0232} & 15,8374 \\
\hline
\end{tabular}

Pelo teste de hipótese, através das estatísticas " $\tilde{\mathrm{F}}$ " e “ $\tilde{\mathrm{t}}$ ", temos que a variável explicativa, X1, do modelo linear exerce efetivamente alguma influência sobre a variável dependente, Y1. Neste caso, temos um alto grau de influência, representado pelo $\widetilde{\mathrm{R}}^{2}=0,9766$. Assim obtemos a equação: $\mathrm{Sh}_{\text {aprox }}=0,7438(\operatorname{ReSc} / \mathrm{X})^{0,3674}$, onde $0,7438=\mathrm{e}^{-0,2959}$.

A Tabela 11 será construída a partir da Equação (143), na qual X2, X3, e X4 são as variáveis linearizadas independentes e Y2 é variável dependente.

A Tabela 12 contém as informações necessárias para análise estatística para análise estatística dos parâmetros w, $\zeta, \gamma$ e $\lambda$, com $\widetilde{R}, \tilde{F}$ e $\tilde{\mathrm{t}}$ sendo, respectivamente, o coeficiente de determinação, a distribuição $F$ de probabilidade e a distribuição de probabilidade t de Student. 
Tabela 11. Valores de "Sh" provindos da solução do modelo em referência, via GITT, para determinação de $\mathrm{Sh}_{\text {aprox }}, \operatorname{com} 200 \leq \mathrm{Re} \leq 800,800 \leq \mathrm{Sc} \leq 2500,0,005 \leq \mathrm{Re}_{\mathrm{w}} \leq 0,014$ e $\mathrm{R}_{0}=3.010^{-3} \mathrm{~m}$.

\begin{tabular}{|c|c|c|c|c|c|c|c|c|c|c|}
\hline \multirow[t]{2}{*}{$\mathrm{Sh}_{\text {mod }}$} & \multirow[t]{2}{*}{$\mathrm{Sh}_{\text {aprox }}$} & \multirow{2}{*}{$\frac{\mathrm{Sh}_{\text {mod }}}{\mathrm{Sh}_{\text {aprox }}}-1$} & \multirow[t]{2}{*}{$\mathrm{X}$} & \multirow[t]{2}{*}{$\mathrm{Re}$} & \multirow[t]{2}{*}{$\mathrm{Sc}$} & \multirow[t]{2}{*}{$\mathrm{Re}_{\mathrm{w}}$} & $\ln \left(\frac{\mathrm{Sh}_{\mathrm{mod}}}{\mathrm{Sh}_{\text {aprox }}}-1\right)$ & $\ln (\mathrm{Re})$ & $\ln (\mathrm{Sc})$ & $\ln \left(\mathrm{Re}_{\mathrm{w}}\right)$ \\
\hline & & & & & & & $\mathrm{Y} 2$ & $\mathrm{X} 2$ & $\mathrm{X} 3$ & $\mathrm{X} 4$ \\
\hline 26,919 & 26,059 & 0,033 & 10 & 200 & 800 & 0,005 & $-3,4116$ & 5,298 & 6,685 & $-5,298$ \\
\hline 10,721 & 11,184 & $-0,041$ & 100 & 200 & 800 & 0,005 & - & 5,298 & 6,685 & $-5,298$ \\
\hline 42,730 & 39,605 & 0,079 & 10 & 200 & 2500 & 0,005 & $-2,5396$ & 5,298 & 7,824 & $-5,298$ \\
\hline 19,738 & 16,997 & 0,161 & 100 & 200 & 2500 & 0,005 & $-1,8248$ & 5,298 & 7,824 & $-5,298$ \\
\hline 41,326 & 41,289 & 0,001 & 10 & 700 & 800 & 0,005 & $-7,0134$ & 6,551 & 6,685 & $-5,298$ \\
\hline 15,526 & 17,720 & $-0,124$ & 100 & 700 & 800 & 0,005 & - & 6,551 & 6,685 & $-5,298$ \\
\hline 58,992 & 62,752 & $-0,060$ & 10 & 700 & 2500 & 0,005 & - & 6,551 & 7,824 & $-5,298$ \\
\hline 26,330 & 26,931 & $-0,022$ & 100 & 700 & 2500 & 0,005 & - & 6,551 & 7,824 & $-5,298$ \\
\hline 30,279 & 26,059 & 0,162 & 10 & 200 & 800 & 0,014 & $-1,8205$ & 5,298 & 6,685 & $-4,269$ \\
\hline 15,230 & 11,184 & 0,362 & 100 & 200 & 800 & 0,014 & $-1,0167$ & 5,298 & 6,685 & $-4,269$ \\
\hline 50,361 & 39,605 & 0,272 & 10 & 200 & 2500 & 0,014 & $-1,3035$ & 5,298 & 7,824 & $-4,269$ \\
\hline 33,401 & 16,997 & 0,965 & 100 & 200 & 2500 & 0,014 & $-0,0355$ & 5,298 & 7,824 & $-4,269$ \\
\hline 43,911 & 41,289 & 0,064 & 10 & 700 & 800 & 0,014 & $-2,7567$ & 6,551 & 6,685 & $-4,269$ \\
\hline 19,609 & 17,720 & 0,107 & 100 & 700 & 800 & 0,014 & $-2,2383$ & 6,551 & 6,685 & $-4,269$ \\
\hline 62,720 & 62,752 & $-0,001$ & 10 & 700 & 2500 & 0,014 & - & 6,551 & 7,824 & $-4,269$ \\
\hline 38,132 & 26,931 & 0,416 & 100 & 700 & 2500 & 0,014 & $-0,8773$ & 6,551 & 7,824 & $-4,269$ \\
\hline
\end{tabular}

Tabela 12 . Quadro para análise estatística dos parâmetros $\mathrm{w}, \zeta, \gamma, \lambda$.

\begin{tabular}{l|c}
\hline \multicolumn{2}{c}{ Estatística de regressão } \\
\hline$\widetilde{\mathrm{R}}^{2}$ & 0,8202 \\
$\widetilde{\mathrm{R}}^{2}$ ajustado & 0,7432 \\
Erro padrão & 0,9335 \\
Observações & 11 \\
$\tilde{\mathrm{F}}$ (Calculado) & 10,6513 \\
$\tilde{\mathrm{F}}$ (Tabelado - confiança: 95\%) & 4,3468 \\
$\tilde{\mathrm{t}}$ (Tabelado - confiança: 95\%) & 2,3646 \\
\hline
\end{tabular}

\begin{tabular}{cccc}
\hline & Coeficientes & Erro padrão & $\tilde{\mathrm{t}}$ (Calculado) \\
\hline Interseção & 7,4685 & 6,1994 & 1,2047 \\
X2 & $-1,2132$ & 0,4983 & $-2,4348$ \\
X3 & 1,2695 & 0,5220 & 2,4321 \\
X4 & 2,5607 & 0,5777 & 4,4330 \\
\hline
\end{tabular}


Pelo teste de hipótese, através das estatísticas " $\tilde{\mathrm{F}}$ " e “ $\tilde{\mathrm{t}}$ ", temos que as variáveis explicativas "X2", "X3" e "X4" do modelo linear exercem efetivamente alguma influência sobre a variável dependente "Y2". Neste caso, temos um bom grau de influência, representado pelo $\mathrm{R}^{2}=0,8202$.

A correlação final obtida é dada por:

$$
\mathrm{Sh}=0,7438(\operatorname{ReSc} / \mathrm{X})^{0,3674}\left(1+1751,9133 \operatorname{Re}^{-1,2132} \mathrm{Sc}^{1,2695} \operatorname{Re}_{\mathrm{w}}^{2,5607}\right)
$$

onde $1751,9133=\mathrm{e}^{7,4685}$.

Utilizando a metodologia da Seção (3.3.2) e os intervalos $200 \leq \mathrm{Re} \leq 700$, $0,510^{-2} \leq \mathrm{Re}_{\mathrm{w}} \leq 1,410^{-2}$ e $800 \leq \mathrm{Sc} \leq 2500$, obtemos valores para a taxa assintótica, $\mathrm{l}$, bem como os valores para a taxa assintótica máxima, $\mathbf{1}_{\max }$ (Equação (103)), os quais podem ser vistos na Tabela 13. Observamos que a taxa assintótica, 1 , deve ser sempre menor que a taxa assintótica máxima, $\imath_{\max }$.

Tabela 13. Resultados para a taxa assintótica e a taxa assintótica máxima, a partir dos limites inferiores e superiores dos intervalos para "Re", "Sc" e " $\mathrm{Re}_{\mathrm{w}}$ ", onde $\varepsilon=0,01$.

\begin{tabular}{ccccc}
\hline $\mathrm{Re}$ & $\mathrm{Sc}$ & $\mathrm{Re}_{\mathrm{w}}$ & $\mathrm{l}$ & $\mathrm{l}_{\max }$ \\
\hline 200 & 800 & 0,005 & 0,145 & 0,500 \\
700 & 800 & 0,005 & 0,140 & 0,629 \\
200 & 2500 & 0,005 & 0,115 & 0,196 \\
700 & 2500 & 0,005 & 0,111 & 0,257 \\
200 & 800 & 0,017 & 0,120 & 0,184 \\
700 & 800 & 0,017 & 0,130 & 0,241 \\
200 & 2500 & 0,017 & 0,064 & 0,752 \\
700 & 2500 & 0,017 & 0,087 & 0,102 \\
\hline
\end{tabular}




\section{(2) Correlação de Gilland-Sherwood a partir do problema convectivo-difusivo elíptico.}

A Tabela 14 será construída a partir da Equação (144), na qual X1 e Y1 são as variáveis linearizadas, respectivamente, independente e dependente.

Tabela 14 . Valores de "Sh" provindos da solução do modelo em referência, via GITT, para determinação de $\mathrm{Sh}_{\text {aprox }}, \operatorname{com} \mathrm{R}_{0}=3.010^{-3} \mathrm{~m}, 200 \leq \mathrm{Re} \leq 800,800 \leq \mathrm{Sc} \leq 2500, \mathrm{Re}_{\mathrm{w}}=0,005$ e $\varepsilon=0,01$.

\begin{tabular}{|c|c|c|c|c|c|c|}
\hline $\mathrm{Sh}_{\text {mod }}$ & $\mathrm{X}$ & $\mathrm{Re}$ & $\mathrm{Sc}$ & $\mathrm{Re} \mathrm{Sc} / \mathrm{X}$ & $\ln (\mathrm{Sh})$ & $\ln (\mathrm{Re} \mathrm{Sc} / \mathrm{X})$ \\
\cline { 5 - 7 } & & & & & $\mathrm{Y} 1$ & $\mathrm{X} 1$ \\
\hline 27,6850 & 10 & 200 & 800 & 16000,00 & 3,3209 & 9,6803 \\
\hline 10,9159 & 100 & 200 & 800 & 1600,00 & 2,3902 & 7,3778 \\
\hline 41,6232 & 10 & 200 & 2500 & 50000,00 & 3,7287 & 10,8198 \\
\hline 19,7697 & 100 & 200 & 2500 & 5000,00 & 2,9842 & 8,5172 \\
\hline 40,8513 & 10 & 700 & 800 & 56000,00 & 3,7099 & 10,9331 \\
\hline 15,8012 & 100 & 700 & 800 & 5600,00 & 2,7601 & 8,6305 \\
\hline 49,5432 & 10 & 700 & 2500 & 175000,00 & 3,9028 & 12,0725 \\
\hline 26,3989 & 100 & 700 & 2500 & 17500,00 & 3,2733 & 9,7700 \\
\hline
\end{tabular}

A Tabela 15 contém as informações necessárias para análise estatística dos parâmetros “s” e $\alpha$, com $\widetilde{R}, \tilde{F}$ e $\tilde{t}$ sendo, respectivamente, o coeficiente de determinação, a distribuição F de probabilidade e a distribuição de probabilidade $t$ de Student.

Pelo teste de hipótese, através das estatísticas " $\tilde{\mathrm{F}} "$ e " $\tilde{\mathrm{t}}$ ", temos que a variável explicativa, X1, do modelo linear exerce efetivamente alguma influência sobre a variável dependente, Y1. Neste caso, temos um alto grau de influência, representado pelo $\widetilde{\mathrm{R}}^{2}=0,9583$. Assim obtemos a equação: $\mathrm{Sh}_{\text {aprox }}=0,9938(\operatorname{ReSc} / \mathrm{X})^{0,3357}$, onde $0,9938=\mathrm{e}^{-0,0062}$. 
Tabela 15 . Quadro para análise estatística dos parâmetros “s” e $\alpha$.

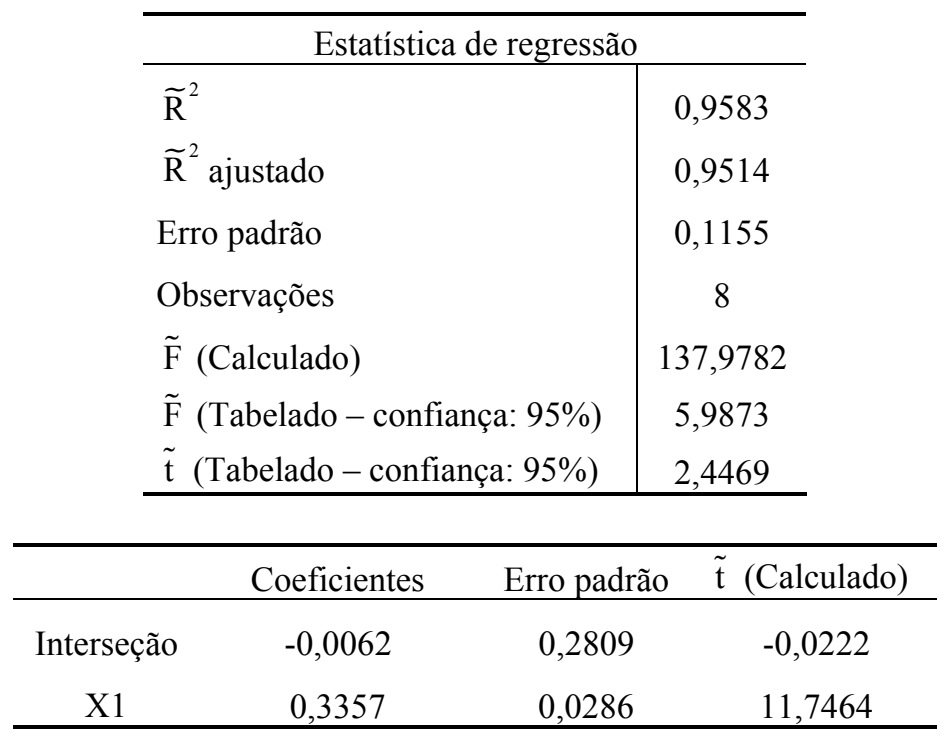

A Tabela 16 será construída a partir da Equação (143), na qual X2, X3, e X4 são as variáveis linearizadas independentes e Y2 é variável dependente.

A Tabela 17 contém as informações necessárias para análise estatística para análise estatística dos parâmetros $\mathrm{w}, \zeta, \gamma$ e $\lambda$, com $\widetilde{\mathrm{R}}, \tilde{\mathrm{F}}$ e $\tilde{\mathrm{t}}$ sendo, respectivamente, o coeficiente de determinação, a distribuição $F$ de probabilidade e a distribuição de probabilidade t de Student.

Pelo teste de hipótese, através das estatísticas " $\tilde{\mathrm{F}}$ " e “ $\tilde{\mathrm{t}}$ ", temos que as variáveis explicativas "X2", "X3" e "X4" do modelo linear exercem efetivamente alguma influência sobre a variável dependente "Y2". Neste caso, temos um bom grau de influência, representado pelo $\mathrm{R}^{2}=0,7912$.

A correlação final obtida é dada por:

$$
\mathrm{Sh}=0,9938(\operatorname{ReSc} / \mathrm{X})^{0,3357}\left(1+2,8546 \operatorname{Re}^{-0,4500} \mathrm{Sc}^{0,6723} \operatorname{Re}_{\mathrm{w}}^{1,1035}\right)
$$

onde $2,8546=\mathrm{e}^{1,0489}$. 
Tabela 16. Valores de "Sh" provindos da solução do modelo em referência, via GITT, para determinação de $\mathrm{Sh}_{\text {aprox }}, \operatorname{com} 200 \leq \mathrm{Re} \leq 800,800 \leq \mathrm{Sc} \leq 2500,0,005 \leq \mathrm{Re}_{\mathrm{w}} \leq 0,014$ e $\mathrm{R}_{0}=3,010^{-3} \mathrm{~m}$.

\begin{tabular}{|c|c|c|c|c|c|c|c|c|c|c|}
\hline \multirow[t]{2}{*}{$\mathrm{Sh}_{\text {mod }}$} & \multirow[t]{2}{*}{$\mathrm{Sh}_{\text {aprox }}$} & \multirow{2}{*}{$\frac{\mathrm{Sh}_{\bmod }}{\mathrm{Sh}_{\text {aprox }}}-1$} & \multirow[t]{2}{*}{$X$} & \multirow[t]{2}{*}{$\mathrm{Re}$} & \multirow[t]{2}{*}{$\mathrm{Sc}$} & \multirow[t]{2}{*}{$\mathrm{Re}_{\mathrm{w}}$} & $\ln \left(\frac{\mathrm{Sh}_{\mathrm{mod}}}{\mathrm{Sh}}-1\right)$ & $\ln (\mathrm{Re})$ & $\ln (\mathrm{Sc})$ & $\ln \left(\mathrm{Re}_{\mathrm{w}}\right)$ \\
\hline & & & & & & & Y2 & $\mathrm{X} 2$ & $\mathrm{X} 3$ & $\mathrm{X} 4$ \\
\hline 27,6850 & 25,6289 & 0,0802 & 10 & 200 & 800 & 0,005 & $-2,523$ & 5,298 & 6,685 & $-5,298$ \\
\hline 10,9159 & 11,8305 & $-0,0773$ & 100 & 200 & 800 & 0,005 & - & 5,298 & 6,685 & $-5,298$ \\
\hline 41,6232 & 37,5721 & 0,1078 & 10 & 200 & 2500 & 0,005 & $-2,227$ & 5,298 & 7,824 & $-5,298$ \\
\hline 19,7697 & 17,3436 & 0,1399 & 100 & 200 & 2500 & 0,005 & $-1,967$ & 5,298 & 7,824 & $-5,298$ \\
\hline 40,8513 & 39,0291 & 0,0467 & 10 & 700 & 800 & 0,005 & $-3,064$ & 6,551 & 6,685 & $-5,298$ \\
\hline 15,8012 & 18,0161 & $-0,1229$ & 100 & 700 & 800 & 0,005 & - & 6,551 & 6,685 & $-5,298$ \\
\hline 49,5432 & 57,2168 & $-0,1341$ & 10 & 700 & 2500 & 0,005 & - & 6,551 & 7,824 & $-5,298$ \\
\hline 26,3989 & 26,4117 & $-0,0005$ & 100 & 700 & 2500 & 0,005 & - & 6,551 & 7,824 & $-5,298$ \\
\hline 30,5585 & 25,6289 & 0,1923 & 10 & 200 & 800 & 0,014 & $-1,648$ & 5,298 & 6,685 & $-4,269$ \\
\hline 15,3165 & 11,8305 & 0,2947 & 100 & 200 & 800 & 0,014 & $-1,222$ & 5,298 & 6,685 & $-4,269$ \\
\hline 46,3182 & 37,5721 & 0,2328 & 10 & 200 & 2500 & 0,014 & $-1,458$ & 5,298 & 7,824 & $-4,269$ \\
\hline 32,1513 & 17,3436 & 0,8538 & 100 & 200 & 2500 & 0,014 & $-0,158$ & 5,298 & 7,824 & $-4,269$ \\
\hline 42,4038 & 39,0291 & 0,0865 & 10 & 700 & 800 & 0,014 & $-2,448$ & 6,551 & 6,685 & $-4,269$ \\
\hline 19,7077 & 18,0161 & 0,0939 & 100 & 700 & 800 & 0,014 & $-2,366$ & 6,551 & 6,685 & $-4,269$ \\
\hline 51,3748 & 57,2168 & $-0,1021$ & 10 & 700 & 2500 & 0,014 & - & 6,551 & 7,824 & $-4,269$ \\
\hline 36,6033 & 26,4117 & 0,3859 & 100 & 700 & 2500 & 0,014 & $-0,952$ & 6,551 & 7,824 & $-4,269$ \\
\hline
\end{tabular}

Tabela 17. Quadro para análise estatística dos parâmetros $\mathrm{w}, \zeta, \gamma, \lambda$.

\begin{tabular}{l|c}
\hline \multicolumn{2}{c}{ Estatística de regressão } \\
\hline$\widetilde{\mathrm{R}}^{2}$ & 0,7912 \\
$\widetilde{\mathrm{R}}^{2}$ ajustado & 0,7018 \\
Erro padrão & 0,4555 \\
Observações & 11 \\
$\tilde{\mathrm{F}}$ (Calculado) & 137,9782 \\
$\tilde{\mathrm{F}}$ (Tabelado - confiança: 95\%) & 5,9873 \\
$\tilde{\mathrm{t}}$ (Tabelado - confiança: 95\%) & 2,4469 \\
\hline
\end{tabular}

\begin{tabular}{lccc}
\hline & Coeficientes & Erro padrão & $\tilde{\mathrm{t}}$ (Calculado) \\
\hline Interseção & 1,0489 & 3,0245 & 0,3468 \\
$\mathrm{X} 2$ & $-0,4500$ & 0,2431 & $-1,8511$ \\
$\mathrm{X} 3$ & 0,6723 & 0,2547 & 2,6402 \\
$\mathrm{X} 4$ & 1,1035 & 0,2818 & 3,9155 \\
\hline
\end{tabular}


Utilizando a metodologia da Seção (3.3.2) e os intervalos $200 \leq \mathrm{Re} \leq 700$, $0,510^{-2} \leq \mathrm{Re}_{\mathrm{w}} \leq 1,410^{-2}$ e $800 \leq \mathrm{Sc} \leq 2500$, obtemos valores para a taxa assintótica, 1 , bem como os valores para a taxa assintótica máxima, $\mathbf{1}_{\max }$ (Equação (103)), os quais podem ser vistos na Tabela 18. Observamos que a taxa assintótica, 1 , deve ser sempre menor que a taxa assintótica máxima, $\imath_{\max }$.

Tabela 18. Resultados para a taxa assintótica e a taxa assintótica máxima, a partir dos limites inferiores e superiores dos intervalos para "Re", "Sc" e "Re

\begin{tabular}{ccccc}
\hline $\mathrm{Re}$ & $\mathrm{Sc}$ & $\mathrm{Re}_{\mathrm{w}}$ & $\mathrm{\imath}$ & $\mathrm{l}_{\max }$ \\
\hline 200 & 800 & 0,005 & 0,162 & 0,500 \\
700 & 800 & 0,005 & 0,152 & 0,629 \\
200 & 2500 & 0,005 & 0,116 & 0,196 \\
700 & 2500 & 0,005 & 0,128 & 0,257 \\
200 & 800 & 0,017 & 0,144 & 0,184 \\
700 & 800 & 0,017 & 0,158 & 0,241 \\
200 & 2500 & 0,017 & 0,087 & 0,752 \\
700 & 2500 & 0,017 & 0,115 & 0,102 \\
\hline
\end{tabular}





\section{APÊndice 5: Resultados eXPerimentais - Paris et Al. (2002).}

O tubo permeável utilizado neste trabalho experimental tem comprimento $L=1,2 \mathrm{~m}$ e raio $R_{0}=0,003 \mathrm{~m}$ e neste processo considerou-se $\rho=1,010^{3} \mathrm{~kg} \mathrm{~m}^{-3}$ e $\mu=1,010^{-3} \mathrm{~kg} \mathrm{~m}^{-1} \mathrm{~s}^{-1}$. Tabela 19 relaciona o fluxo médio experimental, $\mathrm{J}_{\mathrm{m} \text {,exp }}$, às concentrações de entrada, $\mathrm{c}_{0}$, às velocidades média de entrada, $\mathrm{u}_{0}$, e às pressões transmembrana. Nas duas últimas colunas temos X5 e Y3 sendo, respectivamente, a variável independente e a variável dependente relacionadas ao modelo de resistência em série.

Tabela 19. Dados experimentais (PARIS et al., 2002).

\begin{tabular}{cccccc}
\cline { 4 - 5 } & & & & $\left(1 / \mathrm{J}_{\mathrm{m}, \mathrm{exp}}\right) \times 10^{-6}$ & $(1 / \Delta \mathrm{p}) \times 10^{5}$ \\
\hline $\mathrm{c}_{0}(\mathrm{~g} / \mathrm{l})$ & $\mathrm{u}_{0}(\mathrm{~m} / \mathrm{s})$ & $\Delta \mathrm{p} \mathrm{x} 10^{-5}(\mathrm{~Pa})$ & $\mathrm{J}_{\mathrm{m}, \mathrm{xxp}} \times 10^{6}(\mathrm{~m} / \mathrm{s})$ & $\mathrm{Y} 3$ & $\mathrm{X} 5$ \\
\hline 8 & 0,120 & 0,5 & 0,69 & 1,45 & 2,00 \\
8 & 0,120 & 0,9 & 0,85 & 1,18 & 1,11 \\
8 & 0,120 & 2,5 & 1,04 & 0,96 & 0,40 \\
8 & 0,120 & 3,8 & 1,06 & 0,94 & 0,26 \\
\hline 8 & 0,216 & 0,5 & 0,82 & 1,22 & 2,00 \\
8 & 0,216 & 0,9 & 1,09 & 0,92 & 1,11 \\
8 & 0,216 & 2,5 & 1,26 & 0,80 & 0,40 \\
8 & 0,216 & 3,8 & 1,24 & 0,81 & 0,26 \\
8 & 0,216 & 4,9 & 1,24 & 0,80 & 0,20 \\
\hline 8 & 0,312 & 0,5 & 1,06 & 0,94 & 2,00 \\
8 & 0,312 & 2,5 & 1,47 & 0,68 & 0,40 \\
8 & 0,312 & 3,8 & 1,49 & 0,67 & 0,26 \\
8 & 0,312 & 4,9 & 1,58 & 0,63 & 0,20 \\
\hline
\end{tabular}


A partir das duas últimas colunas da Tabela 19 determinamos a constante de proporcionalidade, $\phi$, e a resistência do entupimento da membrana, $\mathrm{R}_{\mathrm{e}}$, através do modelo de resistência em série, Equação (42), e utilizando o método de regressão linear para a equação da reta:

$$
\frac{1}{\mathrm{~J}_{\mathrm{m}, \exp }}=\left(\mathrm{R}_{\mathrm{m}}+\mathrm{R}_{\mathrm{e}}\right) \frac{1}{\Delta \mathrm{p}}+\phi
$$

$\operatorname{com}\left(R_{m}+R_{e}\right)$ e $\phi$ sendo, respectivamente, o coeficiente angular e linear da reta e estes resultados podem ser encontrados na Tabela 20. A velocidade de permeação, $\mathrm{v}_{\mathrm{w}}$, é calculada a partir do modelo de resistência em série. Os adimensionais " $\mathrm{Re}_{\mathrm{w}}$ " e "Re" também estão indicados na Tabela 20 e o adimensional de Schmidt foi considerado $\mathrm{Sc}=2500$.

Tabela 20. Resultados provindos da regressão linear, para a Equação (147) e Tabela 19.

\begin{tabular}{cccccccc}
\hline $\begin{array}{c}\mathrm{u}_{0} \\
(\mathrm{~m} / \mathrm{s})\end{array}$ & $\mathrm{Re}$ & $\begin{array}{c}\phi \times 10^{-5} \\
(\mathrm{~s} / \mathrm{m})\end{array}$ & $\begin{array}{c}\left(\mathrm{R}_{\mathrm{m}}+\mathrm{R}_{\mathrm{e}}\right) \times 10^{-10} \\
\left(\mathrm{~Pa} \mathrm{~s} \mathrm{~m}^{-1}\right)\end{array}$ & $\begin{array}{c}\Delta \mathrm{p} \times 10^{-5} \\
(\mathrm{~Pa})\end{array}$ & $\begin{array}{c}\mathrm{v}_{\mathrm{w}} \times 10^{6} \\
(\mathrm{~m} / \mathrm{s})\end{array}$ & $\mathrm{Re}_{\mathrm{w}}$ & $\begin{array}{c}\mathrm{J}_{\mathrm{m}, \mathrm{exp}} \times 10^{6} \\
(\mathrm{~m} / \mathrm{s})\end{array}$ \\
\hline 0,120 & 360 & 7,97 & 2,46 & 0,5 & 0,78 & 0,0023 & 0,69 \\
0,216 & 648 & 6,87 & 2,67 & 0,5 & 0,82 & 0,0025 & 0,82 \\
0,312 & 936 & 6,35 & 2,83 & 0,5 & 0,83 & 0,0025 & 1,06 \\
\hline 0,120 & 360 & 7,97 & 2,46 & 0,9 & 0,93 & 0,0028 & 0,85 \\
0,216 & 648 & 6,87 & 2,67 & 0,9 & 1,02 & 0,0031 & 1,09 \\
\hline 0,120 & 360 & 7,97 & 2,46 & 2,5 & 1,12 & 0,0034 & 1,04 \\
0,216 & 648 & 6,87 & 2,67 & 2,5 & 1,26 & 0,0038 & 1,26 \\
0,312 & 936 & 6,35 & 2,83 & 2,5 & 1,34 & 0,0040 & 1,47 \\
\hline 0,120 & 360 & 7,97 & 2,46 & 3,8 & 1,16 & 0,0035 & 1,06 \\
0,216 & 648 & 6,87 & 2,67 & 3,8 & 1,32 & 0,0040 & 1,24 \\
0,312 & 936 & 6,35 & 2,83 & 3,8 & 1,41 & 0,0042 & 1,49 \\
\hline 0,216 & 648 & 6,87 & 2,67 & 4,9 & 1,35 & 0,0040 & 1,24 \\
0,312 & 936 & 6,35 & 2,83 & 4,9 & 1,44 & 0,0043 & 1,58 \\
\hline
\end{tabular}




\section{Apêndice 6: Programas}

Apresetação, na forma de CD-Rom, das implementações dos modelos matemáticos apresentados no Capítulo (3), a saber: (1) AutoVal_IntNorm.pdf, (2) CoefIntegraEliptico.pdf, (3) CoefIntegraParabolico.pdf, (4) ProgPrincEliptico.pdf e (5) ProgPrincParabolico.pdf. 
